\title{
Graded Medial $n$-Ary Algebras and Polyadic Tensor Categories
}

\author{
Steven Duplij
}

check for

updates

Citation: Duplij, S. Graded Medial n-Ary Algebras and Polyadic Tensor Categories. Symmetry 2021, 13, 1038. https://doi.org/10.3390/ sym 13061038

Academic Editor: Tomohiro Inagaki

Received: 8 May 2021

Accepted: 3 June 2021

Published: 9 June 2021

Publisher's Note: MDPI stays neutral with regard to jurisdictional claims in published maps and institutional affiliations.

Copyright: (C) 2021 by the author. Licensee MDPI, Basel, Switzerland. This article is an open access article distributed under the terms and conditions of the Creative Commons Attribution (CC BY) license (https:// creativecommons.org/licenses/by/ $4.0 /)$.
Center for Information Technology (WWU IT), Universität Münster, Röntgenstrasse 7-13, 48149 Münster, Germany; douplii@uni-muenster.de

\begin{abstract}
Algebraic structures in which the property of commutativity is substituted by the mediality property are introduced. We consider (associative) graded algebras and instead of almost commutativity (generalized commutativity or $\varepsilon$-commutativity), we introduce almost mediality ("commutativity-to-mediality" ansatz). Higher graded twisted products and "deforming" brackets (being the medial analog of Lie brackets) are defined. Toyoda's theorem which connects (universal) medial algebras with abelian algebras is proven for the almost medial graded algebras introduced here. In a similar way we generalize tensor categories and braided tensor categories. A polyadic (non-strict) tensor category has an $n$-ary tensor product as an additional multiplication with $n-1$ associators of the arity $2 n-1$ satisfying a $\left(n^{2}+1\right)$-gon relation, which is a polyadic analog of the pentagon axiom. Polyadic monoidal categories may contain several unit objects, and it is also possible that all objects are units. A new kind of polyadic categories (called groupal) is defined: they are close to monoidal categories but may not contain units: instead the querfunctor and (natural) functorial isomorphisms, the quertors, are considered (by analogy with the querelements in $n$-ary groups). The arity-nonreducible $n$-ary braiding is introduced and the equation for it is derived, which for $n=2$ coincides with the Yang-Baxter equation. Then, analogously to the first part of the paper, we introduce "medialing" instead of braiding and construct "medialed" polyadic tensor categories.
\end{abstract}

Keywords: grading; commutativity; mediality; tensor category; monoidal category; braiding; Toyoda's theorem

MSC: 16T25; 17A42; 20N15; 20F36; 16E50; 16U80; 18D10; 18D35; 19D23

\section{Introduction}

The commutativity property and its "breaking" are quite obvious and unique for binary algebraic structures, because the permutation group $S_{2}$ has only one non-identity element. If the operation is $n$-ary, however, then one has $n !-1$ non-identity permutations from $S_{n}$, and the uniqueness is lost. The standard way to bring uniqueness to an $n$-ary structure is by restricting to a particular $n$-ary commutation by fixing one chosen permutation using external (sometimes artificial) criteria (e.g., in Leibniz derivations or $J B^{*}$-triples). We introduce a different, canonical approach: to use another property which would be unique by definition but which can give commutativity in special cases. Mediality [1] (acting on $n^{2}$ elements) is such a property which can be substituted for commutativity (acting on $n$ elements) in the generators/relations description of $n$-ary structures. For $n=2$, mediality means $(a b)(c d)=(a c)(b d)$, and any medial magma is a commutative monoid; moreover, for binary groups commutativity immediately follows from mediality.

In the first part of our paper we consider $n$-ary graded algebras and propose the following idea: instead of considering the non-unique commutativity property and its "breaking", to investigate the unique property of mediality and its "breaking"we exploit this "commutativity-to-mediality" ansatz to introduce and study almost medial $n$-ary graded algebras by analogy with almost commutative algebras. By almost we refer to generalized or $\mathcal{\varepsilon}$-commutative graded algebras [2,3] and $\beta$-commutative algebras [4] (see, also [5-7]). For almost medial $n$-ary graded algebras, we prove an analogue of Toyoda's theorem, 
which originally connected medial algebras with abelian algebras [8]. Note that almost comediality for polyadic bialgebras was introduced earlier in [9]. For other (binary) generalizations of grading, see, e.g., [10-12].

The second part of the paper is devoted to a similar consideration of tensor categories $[13,14]$. We define polyadic tensor categories by considering an $n$-ary tensor product (which may not be iterated from binary tensor products) and $n$-ary coherence conditions for the corresponding associators. The peculiarities of polyadic semigroupal and monoidal categories are studied and the differences from the corresponding binary tensor categories are outlined. We introduce a new kind of tensor categories, polyadic nonunital "groupal" categories, which contain a "querfunctor" and "quertors" (similar to querelements in $n$-ary groups $[15,16])$. We introduce arity-nonreducible $n$-ary braidings and find the equation for them that in the binary case turns into the Yang-Baxter equation in the tensor product form. Finally, we apply the "commutativity-to-mediality" ansatz to braided tensor categories [17] and introduce "medialing" and corresponding "medialed" tensor categories.

The proposed "commutativity-to-mediality" ansatz can lead to medial $n$-ary superalgebras and Lie superalgebras as well as to a medial analog of noncommutative geometry.

\section{Preliminaries}

The standard way to generalize the commutativity is using graded vector spaces and corresponding algebras together with the commutation factor defined on some abelian grading group (see, e.g., $[2,3,18,19])$. First, recall this concept from a slightly different viewpoint.

\subsection{Binary Graded Algebra}

Let $\mathscr{A} \equiv \mathscr{A}^{(2)}=\left\langle A \mid \mu_{2}, v_{2} ; \lambda_{1}\right\rangle$ be an associative (binary) algebra over a field $\mathbb{k}$ (having unit $1 \in \mathbb{k}$ and zero $0 \in \mathbb{k}$ ) with unit $e$ (i.e., it is a unital $\mathbb{k}$-algebra) and zero $z \in A$. Here $A$ is the underling set and $\mu_{2}: A \otimes A \rightarrow A$ is the (bilinear) binary multiplication (which we write as $\mu_{2}[a, b], a, b \in A$ ), usually in the binary case denoted by $\operatorname{dot} \mu_{2} \equiv(\cdot)$, and $v_{2}: A \otimes A \rightarrow A$ is the (binary) addition denoted by (+); a third (linear) operation $\lambda_{1}$ is the action $\lambda_{1}: K \otimes A \rightarrow A$ (widely called a "scalar multiplication", but this is not always true, as can be seen from the polyadic case [20]).

Informally, if $\mathscr{A}$ as a vector space can be decomposed into a direct sum, then one can introduce the binary gradation concept: each element $a \in A$ is endowed by an additional characteristic, its gradation denoted by a prime $a^{\prime}$ showing to which subspace it belongs, such that $a^{\prime}$ belongs to a discrete abelian group (initially $\mathbb{N}$ simply to "enumerate" the subspaces, and this can be further generalized to a commutative semigroup). This group is called the binary grading group $\mathscr{G}=\left\langle G, v_{2}^{\prime}\right\rangle$, and usually its operation is written as plus $v_{2}^{\prime} \equiv\left(+^{\prime}\right)$ and the neutral element by $0^{\prime}$. Denote the subset of homogeneous elements of degree $a^{\prime} \in G$ by $A_{a^{\prime}}[3,21]$.

Definition 1. An associative algebra $\mathscr{A}$ is called a binary graded algebra over $\mathbb{k}$ (or G-algebra $\mathscr{A}_{G}$ ), if the algebra multiplication $\mu_{2}$ respects the gradation, i.e.,

$$
\mu_{2}\left[A_{a^{\prime}}, A_{b^{\prime}}\right] \equiv A_{a^{\prime}} \cdot A_{b^{\prime}} \subseteq A_{a^{\prime}+b^{\prime}}, \quad \forall a^{\prime}, b^{\prime} \in G,
$$

where equality corresponds to strong gradation.

If there exist invertible elements of each degree $a^{\prime} \in G$, then $\mathscr{A}$ is called a cross product, and if all non-zero homogeneous elements are invertible, $\mathscr{A}$ is a graded division algebra [21]. Homogeneous (binary) morphisms $\varphi: \mathscr{A}_{G} \rightarrow \mathscr{B}_{G}$ preserve the grading $\varphi\left(A_{a^{\prime}}\right) \subset B_{a^{\prime}}, \forall a^{\prime} \in G$, and the kernel of $\varphi$ is an homogeneous ideal. The corresponding class of $G$-algebras and the homogeneous morphisms form a category of $G$-algebras $G$-Alg (for details, see, e.g., $[18,21]$ ). 


\subsection{Almost Commutativity}

The graded algebras have a rich multiplicative structure, because of the possibility to deform (or twist) the algebra product $\mu_{2}$ by a function depending on the gradation. Let us consider the twisting function (twist factor) $\tau: G \times G \rightarrow \mathbb{k}$.

Definition 2. A twisted graded product $\mu_{2}^{(\tau)}$ is defined for homogeneous elements by

$$
\mu_{2}^{(\tau)}[a, b]=\tau\left(a^{\prime}, b^{\prime}\right) \mu_{2}[a, b], \quad a, b \in A ; \quad a^{\prime}, b^{\prime} \in G .
$$

Proposition 1. If the twisted algebra $\left\langle A \mid \mu_{2}^{(\tau)}\right\rangle$ is associative, then the twisting function becomes a 2-cocycle $\tau \mapsto \sigma: G \times G \rightarrow \mathbb{k}^{\times}$on the abelian group $G$ satisfying

$$
\sigma\left(a^{\prime}, b^{\prime}\right) \sigma\left(a^{\prime}+b^{\prime}, c^{\prime}\right)=\sigma\left(a^{\prime}, b^{\prime}+c^{\prime}\right) \sigma\left(b^{\prime}, c^{\prime}\right), \quad a^{\prime}, b^{\prime}, c^{\prime} \in G
$$

Proof. The result follows from the binary associativity condition for $\mu_{2}^{(\sigma)}$.

Example 1. An example of a solution to the functional Equation (3) is $\sigma\left(a^{\prime}, b^{\prime}\right)=\left(\exp \left(a^{\prime}\right)\right)^{b^{\prime}}$.

The classes of $\sigma$ form the (Schur) multiplier group [3] and for further properties of $\sigma$ and a connection with the cohomology classes $H^{2}(G, \mathbb{k})$, see, e.g., [6].

In general, the twisted product (2) can be any polynomial in algebra elements. Nevertheless, the special cases where $\mu_{2}^{\left(\varepsilon_{0}\right)}[a, b]$ becomes a fixed expression for elements $a, b \in A$ are important.

Definition 3. If the twisted product coincides with the opposite product for all $a, b \in A$, we call the twisting function a 0-level commutation factor $\tau \mapsto \varepsilon_{0}: G \times G \rightarrow \mathbb{k}^{\times}$, such that

$$
\mu_{2}^{\left(\varepsilon_{0}\right)}[a, b]=\mu_{2}[b, a], \text { or } \varepsilon_{0}\left(a^{\prime}, b^{\prime}\right) a \cdot b=b \cdot a, \quad \forall a, b \in A, \quad a^{\prime}, b^{\prime} \in G .
$$

Definition 4. A binary algebra $\mathscr{A}_{2}^{\left(\varepsilon_{0}\right)}$ for which the twisted product coincides with the opposite product $(4)$ is called 0-level almost commutative ( $\varepsilon_{0}$-commutative).

Assertion 1. If the algebra for which (4) takes place is associative, the 0-level commutation factor $\varepsilon_{0}$ satisfies the relations

$$
\begin{aligned}
\varepsilon_{0}\left(a^{\prime}, b^{\prime}\right) \varepsilon_{0}\left(b^{\prime}, a^{\prime}\right) & =1, \\
\varepsilon_{0}\left(a^{\prime}+b^{\prime}, c^{\prime}\right) & =\varepsilon_{0}\left(a^{\prime}, c^{\prime}\right) \varepsilon_{0}\left(b^{\prime}, c^{\prime}\right), \\
\varepsilon_{0}\left(a^{\prime}, b^{\prime}+c^{\prime}\right) & =\varepsilon_{0}\left(a^{\prime}, b^{\prime}\right) \varepsilon_{0}\left(a^{\prime}, c^{\prime}\right), \quad a^{\prime}, b^{\prime}, c^{\prime}, d^{\prime} \in G .
\end{aligned}
$$

Proof. The first relation (5) follows from permutation in (4) twice. The next ones follow from permutation in two ways: for (6) $a \cdot b \cdot c \mapsto a \cdot c \cdot b \mapsto c \cdot a \cdot b$ and $(a \cdot b) \cdot c \mapsto c \cdot(a \cdot b)$, and for (7) $a \cdot b \cdot c \mapsto b \cdot a \cdot c \mapsto b \cdot c \cdot a$ and $a \cdot(b \cdot c) \mapsto(b \cdot c) \cdot a$, using (4).

In a more symmetric form this is

$$
\varepsilon_{0}\left(a^{\prime}+b^{\prime}, c^{\prime}+d^{\prime}\right)=\varepsilon_{0}\left(a^{\prime}, c^{\prime}\right) \varepsilon_{0}\left(b^{\prime}, c^{\prime}\right) \varepsilon_{0}\left(a^{\prime}, d^{\prime}\right) \varepsilon_{0}\left(b^{\prime}, d^{\prime}\right)
$$

The following general expression

$$
\varepsilon_{0}\left(\sum_{i_{a}=1}^{j_{a}} a_{i_{a}}^{\prime} \sum_{i_{b}=1}^{j_{b}} b_{i_{b}}^{\prime}\right)=\prod_{i_{a}=1}^{j_{a}} \prod_{i_{b}=1}^{j_{b}} \varepsilon_{0}\left(a_{i_{a}}^{\prime}, b_{i_{b}}^{\prime}\right), \quad a_{i_{a}}^{\prime}, b_{i_{b}}^{\prime} \in G, \quad i_{a}, i_{b}, j_{a}, j_{b} \in \mathbb{N},
$$

can be written. In the case of equal elements we have 


$$
\varepsilon_{0}\left(j_{a} a^{\prime}, j_{b} b^{\prime}\right)=\left(\varepsilon_{0}\left(a^{\prime}, b^{\prime}\right)\right)^{j_{a} j_{b}} .
$$

Remark 1. Recall that the standard commutation factor $\varepsilon: G \times G \rightarrow \mathbb{k}^{\times}$of an almost commutative ( $\varepsilon$-commutative or $\varepsilon$-symmetric) associative algebra is defined in a different way [2,3]

$$
\varepsilon\left(a^{\prime}, b^{\prime}\right) b \cdot a=a \cdot b
$$

Comparing with (4) we have

$$
\varepsilon_{0}\left(a^{\prime}, b^{\prime}\right)=\varepsilon\left(b^{\prime}, a^{\prime}\right), \quad \forall a^{\prime}, b^{\prime} \in G .
$$

\subsection{Tower of Higher Level Commutation Brackets}

Let us now construct the tower of higher level commutation factors and brackets using the following informal reasoning. We "deform" the almost commutativity relation (4) by a function $L_{0}: A \times A \rightarrow A$ as

$$
\varepsilon_{0}\left(a^{\prime}, b^{\prime}\right) a \cdot b=b \cdot a+L_{0}^{\left(\varepsilon_{0}\right)}(a, b), \quad \forall a, b \in A, \quad a^{\prime}, b^{\prime} \in G,
$$

where $\varepsilon_{0}\left(a^{\prime}, b^{\prime}\right)$ is the 0-level commuting factor satisfying (5)-(7).

Consider the function (bracket) $L_{0}^{\left(\varepsilon_{0}\right)}(a, b)$ as a multiplication of a new algebra

$$
\mathscr{A}_{2}^{L_{0}}=\left\langle A \mid \mu_{2}^{\left(\varepsilon_{0}, L_{0}\right)}=L_{0}^{\left(\varepsilon_{0}\right)}(a, b)\right\rangle
$$

called a 0-level bracket algebra. Then (13) can be treated as its "representation" by the associative algebra $\mathscr{A}$.

Proposition 2. The algebra $\mathscr{A}_{2}^{L_{0}}$ is almost commutative with the commutation factor $\left(-\varepsilon_{0}^{-1}\right)$.

Proof. Using (13) and (5)-(7) we get

$$
\varepsilon_{0}\left(b^{\prime}, a^{\prime}\right) L_{0}^{\left(\varepsilon_{0}\right)}(a, b)+L_{0}^{\left(\varepsilon_{0}\right)}(b, a)=0,
$$

which can be rewritten in the almost commutativity form (4) as

$$
\left(-\varepsilon_{0}\left(b^{\prime}, a^{\prime}\right)\right) L_{0}^{\left(\varepsilon_{0}\right)}(a, b)=L_{0}^{\left(\varepsilon_{0}\right)}(b, a) .
$$

It follows from (5) that

$$
\left(-\varepsilon_{0}^{-1}\left(a^{\prime}, b^{\prime}\right)\right) L_{0}^{\left(\varepsilon_{0}\right)}(a, b)=L_{0}^{\left(\varepsilon_{0}\right)}(b, a)
$$

The triple identity for $L_{0}^{\left(\varepsilon_{0}\right)}(a, b)$ can be obtained using (5)-(7), (13) and (15)

$$
\begin{aligned}
& \varepsilon_{0}\left(c^{\prime}, a^{\prime}\right) L_{0}^{\left(\varepsilon_{0}\right)}\left(L_{0}^{\left(\varepsilon_{0}\right)}(a, b), c\right)+\varepsilon_{0}\left(a^{\prime}, b^{\prime}\right) L_{0}^{\left(\varepsilon_{0}\right)}\left(L_{0}^{\left(\varepsilon_{0}\right)}(b, c), a\right) \\
& +\varepsilon_{0}\left(b^{\prime}, c^{\prime}\right) L_{0}^{\left(\varepsilon_{0}\right)}\left(L_{0}^{\left(\varepsilon_{0}\right)}(c, a), b\right)=0, \quad \forall a, b, c \in A, \quad a^{\prime}, b^{\prime}, c^{\prime} \in G .
\end{aligned}
$$

In the more symmetric form using (8) we have

$$
\begin{aligned}
& \varepsilon_{0}\left(c^{\prime}, b^{\prime}\right) \varepsilon_{0}\left(d^{\prime}, a^{\prime}\right) L_{0}^{\left(\varepsilon_{0}\right)}\left(L_{0}^{\left(\varepsilon_{0}\right)}(a, b), L_{0}^{\left(\varepsilon_{0}\right)}(c, d)\right)+\varepsilon_{0}\left(d^{\prime}, c^{\prime}\right) \varepsilon_{0}\left(a^{\prime}, b^{\prime}\right) L_{0}^{\left(\varepsilon_{0}\right)}\left(L_{0}^{\left(\varepsilon_{0}\right)}(b, c), L_{0}^{\left(\varepsilon_{0}\right)}(d, a)\right) \\
& \varepsilon_{0}\left(a^{\prime}, d^{\prime}\right) \varepsilon_{0}\left(b^{\prime}, c^{\prime}\right) L_{0}^{\left(\varepsilon_{0}\right)}\left(L_{0}^{\left(\varepsilon_{0}\right)}(c, d), L_{0}^{\left(\varepsilon_{0}\right)}(a, b)\right)+\varepsilon_{0}\left(b^{\prime}, a^{\prime}\right) \varepsilon_{0}\left(c^{\prime}, d^{\prime}\right) L_{0}^{\left(\varepsilon_{0}\right)}\left(L_{0}^{\left(\varepsilon_{0}\right)}(d, a), L_{0}^{\left(\varepsilon_{0}\right)}(b, c)\right)=0 .
\end{aligned}
$$


By analogy with (13), we successively further "deform" (15) then introduce "deforming" functions and higher level commutation factors in the following way.

Definition 5. The k-level commutation factor $\varepsilon_{k}\left(a^{\prime}, b^{\prime}\right)$ is defined by the following "differencelike"ẽquations

$$
\begin{aligned}
\varepsilon_{1}\left(a^{\prime}, b^{\prime}\right) L_{0}^{\left(\varepsilon_{0}\right)}(a, b) & =L_{0}^{\left(\varepsilon_{0}\right)}(b, a)+L_{1}^{\left(\varepsilon_{0}, \varepsilon_{1}\right)}(a, b), \\
\varepsilon_{2}\left(a^{\prime}, b^{\prime}\right) L_{1}^{\left(\varepsilon_{0}, \varepsilon_{1}\right)}(a, b) & =L_{1}^{\left(\varepsilon_{0}, \varepsilon_{1}\right)}(b, a)+L_{2}^{\left(\varepsilon_{0}, \varepsilon_{1}, \varepsilon_{2}\right)}(a, b), \\
\vdots & \\
\varepsilon_{k}\left(a^{\prime}, b^{\prime}\right) L_{k-1}^{\left(\varepsilon_{0}, \varepsilon_{1}, \ldots, \varepsilon_{k-1}\right)}(a, b) & =L_{k-1}^{\left(\varepsilon_{0}, \varepsilon_{1}, \ldots, \varepsilon_{k-1}\right)}(b, a)+L_{k}^{\left(\varepsilon_{0}, \varepsilon_{1}, \ldots, \varepsilon_{k}\right)}(a, b) .
\end{aligned}
$$

Definition 6. k-level almost commutativity is defined by the vanishing of the last "deforming" function

$$
L_{k}^{\left(\varepsilon_{0}, \varepsilon_{1}, \ldots, \varepsilon_{k}\right)}(a, b)=0, \quad \forall a, b \in A,
$$

and can be expressed in a form analogous to (4)

$$
\varepsilon_{k}\left(a^{\prime}, b^{\prime}\right) L_{k-1}^{\left(\varepsilon_{0}, \varepsilon_{1}, \ldots, \varepsilon_{k-1}\right)}(a, b)=L_{k-1}^{\left(\varepsilon_{0}, \varepsilon_{1}, \ldots, \varepsilon_{k-1}\right)}(b, a) .
$$

Proposition 3. All higher level "deforming" functions $L_{i}^{\left(\varepsilon_{0}, \varepsilon_{1}, \ldots, \varepsilon_{i}\right)}, i=1, \ldots, k$ can be expressed through $L_{0}^{\left(\varepsilon_{0}\right)}(a, b)$ from (13) multiplied by a combination of the lower level commutation factors $\varepsilon_{i}\left(a^{\prime}, b^{\prime}\right), i=1, \ldots, k$.

Proof. This follows from Equations (18)-(20).

The first such expressions are

$$
\begin{aligned}
L_{1}^{\left(\varepsilon_{0}, \varepsilon_{1}\right)}(a, b) & =\left[\varepsilon_{1}\left(a^{\prime}, b^{\prime}\right)+\varepsilon_{0}\left(b^{\prime}, a^{\prime}\right)\right] L_{0}^{\left(\varepsilon_{0}\right)}(a, b), \\
L_{2}^{\left(\varepsilon_{0}, \varepsilon_{1}, \varepsilon_{2}\right)}(a, b) & =\left[\varepsilon_{2}\left(a^{\prime}, b^{\prime}\right)\left(\varepsilon_{1}\left(a^{\prime}, b^{\prime}\right)+\varepsilon_{0}\left(b^{\prime}, a^{\prime}\right)\right)+\varepsilon_{1}\left(a^{\prime}, b^{\prime}\right) \varepsilon_{0}\left(b^{\prime}, a^{\prime}\right)+1\right] L_{0}^{\left(\varepsilon_{0}\right)}(a, b),
\end{aligned}
$$

Recall the definition of the $\varepsilon$-Lie bracket [3]

$$
[a, b]_{\varepsilon}=a \cdot b-\varepsilon\left(a^{\prime}, b^{\prime}\right) b \cdot a, \quad \forall a, b \in A, \quad a^{\prime}, b^{\prime} \in G .
$$

Assertion 2. The 0-level "deforming" function $L_{0}^{\left(\varepsilon_{0}\right)}(a, b)$ is the $\varepsilon_{0}$-twisted E-Lie bracket

$$
L_{0}^{\left(\varepsilon_{0}\right)}(a, b)=\varepsilon_{0}\left(a^{\prime}, b^{\prime}\right)[a, b]_{\mathcal{\varepsilon}=\varepsilon_{0}} .
$$

Proof. This follows from (13) and (25).

Remark 2. The relations (16) and (17) are analogs of the $\varepsilon$-Jacobi identity of the $\varepsilon$-Lie algebra [3].

Corollary 1. All higher level "deforming" functions $L_{i}^{\left(\varepsilon_{0}, \varepsilon_{1}, \ldots, \varepsilon_{i}\right)}, i=1, \ldots, k$ can be expressed through the twisted $\varepsilon$-Lie bracket (25) with twisting coefficients. 
In search of a polyadic analog of almost commutativity, we will need some additional concepts, beyond the permutation of two elements (in the binary case), called commutativity, and various sums of permutations (of $n$ elements, in $n$-ary case, which are usually non-unique).

Instead we propose to consider a new concept, polyadic mediality (which gives a unique relation between $n^{2}$ elements in $n$-ary case), as a polyadic inductive generalization of commutativity. We then twist the multiplication by a gradation (as in the binary case above) to obtain the polyadic version of almost commutativity as almost mediality. However, let us first recall the binary and polyadic versions of the mediality property.

\subsection{Medial Binary Magmas and Quasigroups}

The mediality property was introduced as a generalization of the associative law for quasigroups, which are a direct generalization of abelian groups [1]. Other names for mediality are entropicity, bisymmetry, alternaton and abelianness (see, e.g., [22-24]).

Let $\mathscr{M}=\left\langle M \mid \mu_{2}\right\rangle$ be a binary magma (a closed set $M$ with one binary operation $\mu_{2}$ without any additional properties, also called a (Hausmann-Ore) groupoid (which should not be confused with the Brandt groupoid or virtual group).

Definition 7. A (binary) magma $\mathscr{M}$ is called medial, if

$$
\mu_{2}\left[\mu_{2}[a, b], \mu_{2}[c, d]\right]=\mu_{2}\left[\mu_{2}[a, c], \mu_{2}[b, d]\right], \quad \forall a, b, c, d \in M .
$$

Definition 8. We call the product of elements in the r.h.s. of (27) medially symmetric to the l.h.s. product.

Obviously, if a magma $\mathscr{M}$ contains a neutral element (identity) $e \in M$, such that $\mu_{2}[a, e]=\mu_{2}[e, a]=a, \forall a \in M$, then $\mathscr{M}$ is commutative $\mu_{2}[a, b]=\mu_{2}[b, a], \forall a, b \in M$. Therefore, any commutative monoid is an example of a medial magma. Numerous different kinds of magma and their classifications are given in [23]. If a magma $\mathscr{M}$ is cancellative $\left(\mu_{2}[a, b]=\mu_{2}[a, c] \Rightarrow b=c, \mu_{2}[a, c]=\mu_{2}[b, c] \Rightarrow a=b, \forall a, b, c \in M\right)$, it is a binary quasigroup $Q=\left\langle Q \mid \mu_{2}\right\rangle$ for which the equations $\mu_{2}[a, x]=b, \mu_{2}[y, a]=b, \forall a, b \in Q$, have a unique solution [25]. Moreover, as per [26], every medial cancellative magma can be embedded in a medial quasigroup (satisfying (27)), and the reverse statement is also true [27]. For a recent comprehensive review on quasigroups (including medial and $n$-ary ones), see, e.g., [28], and references therein.

The structure of medial quasigroups is determined by the Bruck-Murdoch-Toyoda theorem $[8,29,30]$.

Theorem 1 (Toyoda theorem). Any medial quasigroup $Q_{\text {medial }}=\left\langle Q \mid \mu_{2}\right\rangle$ can be presented in the linear (functional) form

$$
\mu_{2}[a, b]=v_{2}\left[v_{2}[\varphi(a), \psi(b)], c\right]=\varphi(a)+\psi(b)+c, \quad \forall a, b, c \in Q,
$$

where $\left\langle Q \mid v_{2} \equiv(+)\right\rangle$ is an abelian group and $\varphi, \psi: Q \rightarrow Q$ are commuting automophisms $\varphi \circ \psi=\psi \circ \varphi$, and $c \in Q$ is fixed.

If $Q_{\text {medial }}$ has an idempotent element (denoted by 0 ), then

$$
\mu_{2}[a, b]=v_{2}[\varphi(a), \psi(b)]=\varphi(a)+\psi(b), \quad \forall a, b \in Q,
$$

It follows from the Toyoda theorem that medial quasigroups are isotopic to abelian groups, and their structure theories are very close [29,30].

The mediality property (27) for binary semigroups leads to various consequences [31,32]. Indeed, every medial semigroup $\mathcal{S}_{\text {medial }}=\left\langle S \mid \mu_{2}\right\rangle$ is a Putcha semigroup $\left(b \in S^{1} a S^{1} \Rightarrow b^{m} \in\right.$ $\left.S^{1} a^{2} S^{1}, \forall a, b \in S, m \in \mathbb{N}, S^{1}=S \cup\{1\}\right)$, and therefore $\mathcal{S}_{\text {medial }}$ can be decomposed into the 
semilattice $\left(a^{2}=a \wedge a b=b a, \forall a, b \in S\right.$ ) of Archimedean semigroups $(\forall a, b \in S, \exists m, k \in \mathbb{N}$, $\left.a^{m}=S^{1} b S^{1} \wedge b^{k}=S^{1} a S^{1} \wedge a b=b a\right)$. If a medial semigroup $\mathcal{S}_{\text {medial }}$ is left (right) cancellative, $a b=a c \Rightarrow b=c(b a=c a \Rightarrow b=c)$, then it is left (right) commutative $a b c=b a c(a b c=a c b)$, $\forall a, b, c \in S$ and left (right) separative, $a b=a^{2} \wedge b a=b^{2}, \forall a, b \in S\left(a b=b^{2} \wedge b a=a^{2}\right)$ (for a review, see, [33]).

For a binary group $\left\langle G \mid \mu_{2}\right\rangle$ mediality implies commutativity, because, obviously, $a b c d=a c b d \Rightarrow b c=c b, \forall a, b, c, d \in G$. This is not the case for polyadic groups, where mediality implies semicommutativity only (see e.g., [34,35]).

Let $\mathscr{A}=\left\langle A \mid \mu_{2}, v_{2} ; \lambda_{1}\right\rangle$ be a binary $\mathbb{k}$-algebra which is not necessarily unital, cancellative and associative. Then mediality provides the corresponding behavior which depends on the properties of the "vector multiplication" $\mu_{2}$. For instance, for unital cancellative and associative algebras, mediality implies commutativity, as for groups [34].

\section{Almost Medial Binary Graded Algebras}

Consider an associative binary algebra $\mathscr{A}$ over a field $\mathbb{k}$. We introduce a weaker version of gradation than in (1).

Definition 9. An associative algebra $\mathscr{A}$ is called a binary higher graded algebra over $\mathbb{k}$, if the algebra multiplication of four $\left(=2^{2}\right)$ elements respects the gradation

$$
\mu_{4}\left[A_{a^{\prime}}, A_{b^{\prime}}, A_{c^{\prime}}, A_{d^{\prime}}\right] \equiv A_{a^{\prime}} \cdot A_{b^{\prime}} \cdot A_{c^{\prime}} \cdot A_{d^{\prime}} \subseteq A_{a^{\prime}+{ }^{\prime} b^{\prime}+c^{\prime}+d^{\prime}}, \forall a^{\prime}, b^{\prime}, c^{\prime}, d^{\prime} \in G,
$$

where equality corresponds to strong higher gradation.

Instead of (2) let us introduce the higher twisting function (higher twist factor) for four $\left(=2^{2}\right)$ elements $\tau: G^{\times 4} \rightarrow \mathbb{k}$.

Definition 10. A twisted (binary) higher graded product $\boldsymbol{\mu}_{4}^{(\tau)}$ is defined for homogeneous elements by

$$
\mu_{4}^{(\tau)}[a, b, c, d]=\tau\left(a^{\prime}, b^{\prime}, c^{\prime}, d^{\prime}\right) a \cdot b \cdot c \cdot d, \quad a, b, c, d \in A ; \quad a^{\prime}, b^{\prime}, c^{\prime}, d^{\prime} \in G .
$$

An analog of (total) associativity for the twisted binary higher graded product operation $\mu_{4}^{(\tau)}$ is the following condition on seven elements $\left(7=2 \cdot 2^{2}-1\right)$ for all $a, b, c, d, t, u, v \in A$

$$
\begin{aligned}
& \boldsymbol{\mu}_{4}^{(\tau)}\left[\boldsymbol{\mu}_{4}^{(\tau)}[a, b, c, d], t, u, v\right]=\boldsymbol{\mu}_{4}^{(\tau)}\left[a, \boldsymbol{\mu}_{4}^{(\tau)}[b, c, d, t], u, v\right] \\
& =\boldsymbol{\mu}_{4}^{(\tau)}\left[a, b, \boldsymbol{\mu}_{4}^{(\tau)}[c, d, t, u], v\right]=\boldsymbol{\mu}_{4}^{(\tau)}\left[a, b, c, \boldsymbol{\mu}_{4}^{(\tau)}[d, t, u, v]\right] .
\end{aligned}
$$

Proposition 4. If the twisted higher graded product satisfies the higher analog of associativity given by (32), then the twisting function becomes a higher analog of the cocycle (3) $\tau \mapsto \sigma: G^{\times 4} \rightarrow \mathbb{k}^{\times}$ on the abelian group $G$ satisfying for all $a^{\prime}, b^{\prime}, c^{\prime}, d^{\prime}, t^{\prime}, u^{\prime}, v^{\prime} \in G$

$$
\begin{aligned}
& \sigma\left(a^{\prime}, b^{\prime}, c^{\prime}, d^{\prime}\right) \sigma\left(a^{\prime}+b^{\prime}+c^{\prime}+d^{\prime}, t^{\prime}, u^{\prime}, v^{\prime}\right)=\sigma\left(b^{\prime}, c^{\prime}, d^{\prime}, t^{\prime}\right) \sigma\left(a^{\prime}, b^{\prime}+c^{\prime}+d^{\prime}+t^{\prime}, u^{\prime}, v^{\prime}\right) \\
& =\sigma\left(c^{\prime}, d^{\prime}, t^{\prime}, u^{\prime}\right) \sigma\left(a^{\prime}, b^{\prime}, c^{\prime}+d^{\prime}+t^{\prime}+u^{\prime}, v^{\prime}\right)=\sigma\left(d^{\prime}, t^{\prime}, u^{\prime}, v^{\prime}\right) \sigma\left(a^{\prime}, b^{\prime}, c^{\prime}, d^{\prime}+t^{\prime}+u^{\prime}+v^{\prime}\right) .
\end{aligned}
$$

Next we propose a medial analog of almost commutativity as follows. Instead of deforming commutativity by the grading twist factor $\varepsilon_{0}$ as in (4), we deform the mediality (27) by the higher twisting function $\tau$ (31). 
Definition 11. If the higher twisted product coincides with the medially symmetric product (see (27)) for all $a, b \in A$, we call the twisting function a 0-level mediality factor $\tau \mapsto \rho_{0}: G^{\times 4} \rightarrow$ $\mathbb{k}^{\times}$, such that (cf. (4))

$$
\begin{aligned}
\mu_{4}^{\left(\rho_{0}\right)}[a, b, c, d] & =\mu_{4}[a, c, b, d], \text { or } \\
\rho_{0}\left(a^{\prime}, b^{\prime}, c^{\prime}, d^{\prime}\right) a \cdot b \cdot c \cdot d & =a \cdot c \cdot b \cdot d, \quad \forall a, b, c, d \in A, \quad a^{\prime}, b^{\prime}, c^{\prime}, d^{\prime} \in G .
\end{aligned}
$$

From (35) follows the normalization condition for the mediality factor

$$
\rho_{0}\left(a^{\prime}, a^{\prime}, a^{\prime}, a^{\prime}\right)=1, \quad \forall a^{\prime} \in G
$$

Definition 12. A binary algebra $\mathscr{A}_{2}^{\left(\rho_{0}\right)}=\left\langle A \mid \mu_{2}, v_{2}\right\rangle$ for which the higher twisted product coincides with the medially symmetric product $\mu_{4}^{\left(\rho_{0}\right)}[a, b, c, d]=\mu_{4}[a, c, b, d]$ (35) is called a 0-level almost medial ( $\rho_{0}$-commutative) algebra.

Proposition 5. If the algebra for which (35) holds is associative, the 0-level mediality factor $\boldsymbol{\rho}_{0}$ satisfies the relations

$$
\begin{aligned}
& \rho_{0}\left(a^{\prime}, b^{\prime}, c^{\prime}, d^{\prime}\right) \rho_{0}\left(a^{\prime}, c^{\prime}, b^{\prime}, d^{\prime}\right)=1, \quad a^{\prime}, b^{\prime}, c^{\prime}, d^{\prime}, f^{\prime}, g^{\prime}, h^{\prime} \in G, \quad 1 \in \mathbb{k}, \\
& \rho_{0}\left(a^{\prime}, c^{\prime}+d^{\prime}+f^{\prime}+g^{\prime}, b^{\prime}, h^{\prime}\right)=\rho_{0}\left(a^{\prime}, c^{\prime}, b^{\prime}, d^{\prime}\right) \\
& \times \rho_{0}\left(c^{\prime}, d^{\prime}, b^{\prime}, f^{\prime}\right) \rho_{0}\left(d^{\prime}, f^{\prime}, b^{\prime}, g^{\prime}\right) \rho_{0}\left(f^{\prime}, g^{\prime}, b^{\prime}, h^{\prime}\right), \\
& \rho_{0}\left(a^{\prime}, g^{\prime}, b^{\prime}+c^{\prime}+d^{\prime}+f^{\prime}, h^{\prime}\right)=\rho_{0}\left(a^{\prime}, g^{\prime}, b^{\prime} c^{\prime}\right) \\
& \times \rho_{0}\left(c^{\prime}, g^{\prime}, d^{\prime}, f^{\prime}\right) \rho_{0}\left(d^{\prime}, g^{\prime}, f^{\prime}, h^{\prime}\right) \rho_{0}\left(b^{\prime}, g^{\prime}, c^{\prime}, d^{\prime}\right) .
\end{aligned}
$$

Proof. As in (5), the relation (37) follows from applying (35) twice. The next ones follow from permutation in two ways using (34): for (38)

$$
\begin{aligned}
a \cdot b \cdot(c \cdot d \cdot f \cdot g) \cdot h & \mapsto a \cdot(c \cdot d \cdot f \cdot g) \cdot b \cdot h, \quad a, c, d, f, g, b, h \in A, \\
a \cdot b \cdot c \cdot d \cdot f \cdot g \cdot h & \mapsto a \cdot c \cdot b \cdot d \cdot f \cdot g \cdot h \mapsto a \cdot c \cdot d \cdot b \cdot f \cdot g \cdot h \\
& \mapsto a \cdot c \cdot d \cdot f \cdot b \cdot g \cdot h \mapsto a \cdot c \cdot d \cdot f \cdot g \cdot b \cdot h,
\end{aligned}
$$

and for (39)

$$
\begin{aligned}
a \cdot(b \cdot c \cdot d \cdot f) \cdot g \cdot h & \mapsto a \cdot g \cdot(b \cdot c \cdot d \cdot f) \cdot h \\
a \cdot b \cdot c \cdot d \cdot f \cdot g \cdot h & \mapsto a \cdot b \cdot c \cdot d \cdot g \cdot f \cdot h \mapsto a \cdot b \cdot c \cdot g \cdot d \cdot f \cdot h \\
& \mapsto a \cdot b \cdot g \cdot c \cdot d \cdot f \cdot h \mapsto a \cdot g \cdot b \cdot c \cdot d \cdot f \cdot h
\end{aligned}
$$

Assertion 3. If the 0-level almost medial algebra $\mathscr{A}_{2}^{\left(\rho_{0}\right)}$ is cancellative, then it is isomorphic to an almost commutative algebra.

Proof. After cancellation by $a$ and $d$ in (35), we obtain $\varepsilon_{0}\left(b^{\prime}, c^{\prime}\right) b \cdot c=c \cdot b$, where

$$
\varepsilon_{0}\left(b^{\prime}, c^{\prime}\right)=\rho_{0}\left(a^{\prime}, b^{\prime}, c^{\prime}, d^{\prime}\right)
$$

In case $\mathscr{A}_{2}^{\left(\rho_{0}\right)}$ is unital, one can choose $\varepsilon_{0}\left(b^{\prime}, c^{\prime}\right)=\rho_{0}\left(e^{\prime}, b^{\prime}, c^{\prime}, e^{\prime}\right) \equiv \rho_{0}\left(0^{\prime}, b^{\prime}, c^{\prime}, 0^{\prime}\right)$, since the identity $e \in A$ is zero graded. 
Tower of Higher Binary Mediality Brackets

By analogy with (13), let us deform the medial twisted product $\mu_{4}^{\left(\rho_{0}\right)}$ (34) by the function $M_{0}^{\left(\rho_{0}\right)}: A \times A \times A \times A \rightarrow A$ as follows

$$
\rho_{0}\left(a^{\prime}, b^{\prime}, c^{\prime}, d^{\prime}\right) a \cdot b \cdot c \cdot d=a \cdot c \cdot b \cdot d+M_{0}^{\left(\rho_{0}\right)}(a, b, c, d), \quad \forall a, b, c, d \in A, \quad a^{\prime}, b^{\prime}, c^{\prime}, d^{\prime} \in G,
$$

where $\boldsymbol{\rho}_{0}$ is a 0-level mediality factor (34) which satisfies (37)-(39).

Let us next introduce a 4-ary multiplication $\mu_{4}^{\left(\rho_{0}, M_{0}\right)}[a, b, c, d]=M_{0}^{\left(\rho_{0}\right)}(a, b, c, d)$, $\forall a, b, c, d \in A$.

Definition 13. A 4-ary algebra

$$
\mathscr{A}_{4}^{\left(\rho_{0}, M_{0}\right)}=\left\langle A \mid \mu_{4}^{\left(\rho_{0}, M_{0}\right)}\right\rangle
$$

is called a 0-level medial bracket algebra.

Proposition 6. The 4-ary algebra $\mathscr{A}_{4}^{\left(\rho_{0}, M_{0}\right)}$ is almost medial with the mediality factor $\left(-\rho_{0}^{-1}\right)$.

Proof. Using (45) and (37)-(39) we get

$$
\rho_{0}\left(a^{\prime}, c^{\prime}, b^{\prime}, d^{\prime}\right) M_{0}^{\left(\rho_{0}\right)}(a, b, c, d)+M_{0}^{\left(\rho_{0}\right)}(a, c, b, d)=0,
$$

which can be rewritten in the almost medial form (35) as

$$
\left(-\rho_{0}\left(a^{\prime}, c^{\prime}, b^{\prime}, d^{\prime}\right)\right) M_{0}^{\left(\rho_{0}\right)}(a, b, c, d)=M_{0}^{\left(\rho_{0}\right)}(a, c, b, d) .
$$

From (37) we get

$$
\left(-\rho_{0}^{-1}\left(a^{\prime}, b^{\prime}, c^{\prime}, d^{\prime}\right)\right) M_{0}^{\left(\rho_{0}\right)}(a, b, c, d)=M_{0}^{\left(\rho_{0}\right)}(a, c, b, d) .
$$

Let us "deform" (35) again successively by introducing further "deforming" functions $M_{k}$ and higher level mediality factors $\rho_{k}: G \times G \times G \times G \rightarrow \mathbb{k}$ in the following way.

Definition 14. The k-level mediality factor $\boldsymbol{\rho}_{k}\left(a^{\prime}, b^{\prime}, c^{\prime}, d^{\prime}\right)$ is defined by the following "differencelike" equations

$$
\begin{aligned}
\rho_{1}\left(a^{\prime}, b^{\prime}, c^{\prime}, d^{\prime}\right) M_{0}^{\left(\rho_{0}\right)}(a, b, c, d) & =M_{0}^{\left(\rho_{0}\right)}(a, c, b, d)+M_{1}^{\left(\rho_{0}, \rho_{1}\right)}(a, b, c, d), \\
\rho_{2}\left(a^{\prime}, b^{\prime}, c^{\prime}, d^{\prime}\right) M_{1}^{\left(\rho_{0}\right)}(a, b, c, d) & =M_{1}^{\left(\rho_{0}\right)}(a, c, b, d)+M_{2}^{\left(\rho_{0}, \rho_{1}, \rho_{2}\right)}(a, b, c, d), \\
\vdots & \\
\rho_{k}\left(a^{\prime}, b^{\prime}, c^{\prime}, d^{\prime}\right) M_{k-1}^{\left(\rho_{0}, \rho_{1}, \ldots, \rho_{k-1}\right)}(a, b, c, d) & =M_{k-1}^{\left(\rho_{0}, \rho_{1}, \ldots, \rho_{k-1}\right)}(a, c, b, d)+M_{k}^{\left(\rho_{0}, \rho_{1}, \ldots, \rho_{k}\right)}(a, b, c, d), \\
\forall a, b, c, d & \in A, \quad a^{\prime}, b^{\prime}, c^{\prime}, d^{\prime} \in G .
\end{aligned}
$$

Definition 15. k-level almost mediality is defined by the vanishing of the last "deforming" medial function

$$
M_{k}^{\left(\rho_{0}, \rho_{1}, \ldots, \rho_{k}\right)}(a, b, c, d)=0, \quad \forall a, b, c, d \in A,
$$

and can be expressed in a form analogous to (4) and (35)

$$
\rho_{k}\left(a^{\prime}, b^{\prime}, c^{\prime}, d^{\prime}\right) M_{k-1}^{\left(\rho_{0}, \rho_{1}, \ldots, \rho_{k-1}\right)}(a, b, c, d)=M_{k-1}^{\left(\rho_{0}, \rho_{1}, \ldots, \rho_{k-1}\right)}(a, c, b, d) .
$$


Proposition 7. The higher level "deforming" functions $M_{i}^{\left(\rho_{0}, \rho_{1}, \ldots, \rho_{i}\right)}(a, b, c, d), i=1, \ldots, k$ can be expressed through $M_{0}^{\left(\rho_{0}\right)}(a, b, c, d)$ from (45) multiplied by a combination of the lower level mediality factors $\rho_{i}\left(a^{\prime}, b^{\prime}, c^{\prime}, d^{\prime}\right), i=1, \ldots, k$.

Proof. It follows from Equations (48)-(50).

\section{Medial $n$-Ary Algebras}

We now extend the concept of almost mediality from binary to polyadic ( $n$-ary) algebras in the unique way which uses the construction from the previous section.

Let $\mathscr{A}^{(n)}=\left\langle A \mid \mu_{n}, v_{2}\right\rangle$ be an associative $n$-ary algebra (with $n$-ary linear multiplication $A^{\otimes n} \rightarrow A$ ) over a field $\mathbb{k}$ with (possible) polyadic unit $e$ (then $\mathscr{A}^{(n)}$ a unital $\mathbb{k}$-algebra) defined by $\mu_{n}\left[e^{n-1}, a\right]=a, \forall a \in A$ (where $a$ can be on any place) and (binary) zero $z \in A$. We restrict ourselves (as in [36,37]) by the binary addition $v_{2}: A \otimes A \rightarrow A$ which is denoted by $(+)$ (for more general cases, see [20]). Now polyadic (total) associativity [37] can be defined as a kind of invariance [38]

$$
\mu_{n}\left[\boldsymbol{a}, \mu_{n}\left[\boldsymbol{b}_{(n)}\right], \boldsymbol{c}\right]=\text { invariant },
$$

where $\boldsymbol{a}, \boldsymbol{c}$ are (linear) polyads (sequences of elements from $A$ ) of the necessary length [16], $\boldsymbol{b}_{(n)}$ is a polyad of the length $n$, and the internal multiplication can be on any place. To describe the mediality for arbitrary arity $n$ we need the following matrix generalization of polyads (as was implicitly used in [20,38]).

Definition 16. A matrix (n-ary) polyad $\hat{A}_{\left(n^{2}\right)} \equiv \hat{A}_{(n \times n)}$ of size $n \times n$ is the sequence of $n^{2}$ elements $\hat{A}_{(n \times n)}=\left(a_{i j}\right) \in A^{\otimes n^{2}}, i, j=1, \ldots, n$, and their product $\mathrm{A}_{n^{2}}^{(\mu)}: A^{\otimes n^{2}} \rightarrow$ A contains $n+1$ of $n$-ary multiplications $\mu_{n}$, which can be written as (we use that for matrices of arguments, even informally)

$$
\mathrm{A}_{n^{2}}^{(\mu)} \equiv\left(\mu_{n}\right)^{\circ(n+1)}\left[\hat{A}_{\left(n^{2}\right)}\right]=\mu_{n}\left[\begin{array}{c}
\mu_{n}\left[a_{11}, a_{12}, \ldots, a_{1 n}\right], \\
\mu_{n}\left[a_{21}, a_{22}, \ldots, a_{2 n}\right], \\
\vdots \\
\mu_{n}\left[a_{n 1}, a_{n 2}, \ldots, a_{n n}\right]
\end{array}\right] \in A
$$

due to the total associativity (53) (by "omitting brackets").

This construction is the stack reshape of a matrix or row-major order of an array.

Example 2. In terms of matrix polyads the (binary) mediality property (27) becomes

$$
\begin{aligned}
\left(\mu_{2}\right)^{\circ 3}\left[\hat{A}_{(4)}\right] & =\left(\mu_{2}\right)^{\circ 3}\left[\hat{A}_{(4)}^{T}\right], \text { or } \mathrm{A}_{4}^{(\mu)}=\mathrm{A}_{4}^{T(\mu)} \\
\hat{A}_{(4)} & =\left(\begin{array}{ll}
a_{11} & a_{12} \\
a_{21} & a_{22}
\end{array}\right) \Rightarrow\left(a_{11}, a_{12}, a_{21}, a_{22}\right) \in A^{\otimes 4},
\end{aligned}
$$

where $\hat{A}_{(4)}^{T}$ is the transposed polyad matrix representing the sequence $\left(a_{11}, a_{21}, a_{12}, a_{22}\right) \in A^{\otimes 4}$, $\mathrm{A}_{4}^{(\mu)}=\left(\left(a_{11} \cdot a_{12}\right) \cdot\left(a_{21} \cdot a_{22}\right)\right) \in A$ and $\mathbf{A}_{4}^{T(\mu)}=\left(\left(a_{11} \cdot a_{21}\right) \cdot\left(a_{12} \cdot a_{22}\right)\right) \in A$ with $(\cdot) \equiv \mu_{2}$.

Definition 17. A polyadic (n-ary) mediality property is defined by the relation

$$
\begin{aligned}
\left(\mu_{n}\right)^{o n+1}\left[\hat{A}_{\left(n^{2}\right)}\right] & =\left(\mu_{n}\right)^{o n+1}\left[\hat{A}_{\left(n^{2}\right)}^{T}\right], \text { or } \quad \mathrm{A}_{n^{2}}^{(\mu)}=\mathrm{A}_{n^{2}}^{T(\mu)}, \\
\hat{A}_{\left(n^{2}\right)} & =\left(a_{i j}\right) \in A^{\otimes n^{2}} .
\end{aligned}
$$


Definition 18. A polyadic medial twist map $\chi_{\text {medial }}^{\left(n^{2}\right)}$ is defined on the matrix polyads as [9]

$$
\hat{A}_{\left(n^{2}\right)} \stackrel{\chi_{\text {medial }}^{\left(n^{2}\right)}}{\longmapsto} \hat{A}_{\left(n^{2}\right)}^{T} .
$$

Definition 19. A n-ary algebra $\mathscr{A}^{(n)}$ is called medial, if it satisfies the $n$-ary mediality property (57) for all $a_{i j} \in A$.

It follows from (55) that not all medial binary algebras are abelian.

Corollary 2. If a binary medial algebra $\mathscr{A}^{(2)}$ is cancellative, it is abelian.

Assertion 4. If a n-ary medial algebra $\mathscr{A}^{(n)}$ is cancellative, each matrix polyad $\hat{A}_{\left(n^{2}\right)}$ satisfies $n^{2}-2$ commutativity-like relations.

\section{Almost Medial $n$-Ary Graded Algebras}

The gradation for associative $n$-ary algebras was considered in $[36,39]$. Here we introduce a weaker version of gradation, because we need to define the grading twist not for $n$-ary multiplication, i.e., the polyads of the length $n$ but only for the matrix polyads (58) of the length $n^{2}$ (for the binary case, see (30)).

Definition 20. An associative n-ary algebra $\mathscr{A}^{(n)}$ is called a higher graded $n$-ary algebra over $\mathbb{k}$, if the algebra multiplication of $n^{2}$ elements respects the gradation, i.e.,

$$
\begin{aligned}
& \left(\mu_{n}\right)^{\circ n+1}\left[A_{\left(a_{i j}^{\prime}\right)}\right] \equiv \mu_{n}\left[\begin{array}{c}
\mu_{n}\left[A_{a_{11}^{\prime}}, A_{a_{12}^{\prime}}, \ldots, A_{a_{1 n}^{\prime}}\right], \\
\mu_{n}\left[A_{a_{21}^{\prime}}, A_{a_{22}^{\prime}}, \ldots, A_{a_{21}^{\prime}}\right], \\
\vdots \\
\mu_{n}\left[A_{a_{n 1}^{\prime}}, A_{a_{n 2}^{\prime}}, \ldots, A_{a_{n n}^{\prime}}\right]
\end{array}\right] \subseteq A_{a_{11}^{\prime}+\ldots+a_{n n}^{\prime}} \\
& \forall a_{i j}^{\prime} \in G, i, j=1, \ldots, n \text {, }
\end{aligned}
$$

where equality corresponds to strong higher gradation.

Let us define the higher twisting function (higher twist factor) for $n^{2}$ elements $\tau_{n^{2}}$ : $G^{\times n^{2}} \rightarrow \mathbb{k}$ by using matrix polyads (for $n=2$ see (31)).

Definition 21. A n-ary higher graded twisted product $\boldsymbol{\mu}_{n^{2}}^{(\tau)}$ is defined for homogeneous elements by

$$
\boldsymbol{\mu}_{n^{2}}^{(\tau)}\left[\hat{\boldsymbol{A}}_{\left(n^{2}\right)}\right]=\boldsymbol{\tau}_{n^{2}}\left(\hat{A}_{\left(n^{2}\right)}^{\prime}\right) \mathrm{A}_{n^{2}}^{(\mu)}, \quad a_{i j} \in A ; a_{i j}^{\prime} \in G, \quad i, j=1, \ldots, n,
$$

where $\hat{A}_{\left(n^{2}\right)}=\left(a_{i j}\right) \in A^{\otimes n^{2}}$ is the matrix polyad of elements (58), and $\hat{A}_{\left(n^{2}\right)}^{\prime}=\left(a_{i j}^{\prime}\right) \in G^{\otimes n^{2}}$ is the matrix polyad of their gradings.

A medial analog of $n$-ary almost mediality can be introduced in a way analogous to the binary case (35).

Definition 22. If the higher twisted product coincides with the medially symmetric product (see (59)) for all $a_{i j} \in A$, we call the twisting function a 0-level n-ary mediality factor $\tau_{n^{2}} \mapsto \rho_{0}^{\left(n^{2}\right)}$ : $G^{\times n^{2}} \rightarrow \mathbb{k}^{\times}$, such that (cf. (4)) 


$$
\begin{aligned}
\mu_{n^{2}}^{\left(\rho_{0}\right)}\left[\hat{A}_{\left(n^{2}\right)}\right] & =\mathrm{A}_{n^{2}}^{T(\mu)}, \text { or } \\
\rho_{0}^{\left(n^{2}\right)}\left(\hat{A}_{\left(n^{2}\right)}^{\prime}\right) \mathrm{A}_{n^{2}}^{(\mu)} & =\mathrm{A}_{n^{2}}^{T(\mu)}, \quad a_{i j} \in A ; \quad a_{i j}^{\prime} \in G, i, j=1, \ldots, n .
\end{aligned}
$$

It follows from (63) that the normalization condition for the $n$-ary mediality factor is

$$
\rho_{0}^{\left(n^{2}\right)}(\overbrace{a^{\prime}, \ldots, a^{\prime}}^{n^{2}})=1, \quad \forall a^{\prime} \in G .
$$

Assertion 5. The 0-level n-ary mediality factor $\rho_{0}^{\left(n^{2}\right)}$ satisfies

$$
\rho_{0}^{\left(n^{2}\right)}\left(\hat{A}_{\left(n^{2}\right)}^{\prime}\right) \rho_{0}^{\left(n^{2}\right)}\left(\left(\hat{A}_{\left(n^{2}\right)}^{\prime}\right)^{T}\right)=1
$$

Proof. It follows from (63) and its transpose together with the relation $\left(B^{T}\right)^{T}=B$ for any matrix over $\mathbb{k}$.

Definition 23. An n-ary algebra for which the higher twisted product coincides with the medially symmetric product (63), is called a 0-level almost medial ( $\rho_{0}$-commutative) $n$-ary algebra $\mathscr{A}_{n}^{\left(\rho_{0}\right)}$.

Recall [18], that a tensor product of binary algebras can be naturally endowed with a $\varepsilon_{0}$-graded structure in the following way (in our notation). Let $\mathscr{A}_{2}^{\left(\varepsilon_{0}\right)}=\left\langle A \mid \mu_{2}^{(a)}\right\rangle$ and $\mathscr{B}_{2}^{\left(\varepsilon_{0}\right)}=\left\langle A \mid \mu_{2}^{(b)}\right\rangle$ be binary graded algebras with the multiplications $\mu_{2}^{(a)} \equiv(\cdot a)$ and $\mu_{2}^{(b)} \equiv\left({ }_{b}\right)$ and the same commutation factor $\varepsilon_{0}$ (see (4)), that is the same G-graded structure. Consider the tensor product $\mathscr{A}_{2}^{\left(\varepsilon_{0}\right)} \otimes \mathscr{B}_{2}^{\left(\varepsilon_{0}\right)}$ and introduce the total $\varepsilon_{0}$-graded multiplication $\left(\mathscr{A}_{2}^{\left(\varepsilon_{0}\right)} \otimes \mathscr{B}_{2}^{\left(\varepsilon_{0}\right)}\right) \star^{\left(\varepsilon_{0}\right)}\left(\mathscr{A}_{2}^{\left(\varepsilon_{0}\right)} \otimes \mathscr{B}_{2}^{\left(\varepsilon_{0}\right)}\right) \longrightarrow \mathscr{A}_{2}^{\left(\varepsilon_{0}\right)} \otimes \mathscr{B}_{2}^{\left(\varepsilon_{0}\right)}$ defined by the deformation (cf. (2))

$$
\varepsilon_{0}\left(b_{1}^{\prime}, a_{2}^{\prime}\right)\left(a_{1} \otimes b_{1}\right) \star^{\left(\varepsilon_{0}\right)}\left(a_{2} \otimes b_{2}\right), b_{1}^{\prime}, a_{2}^{\prime} \in G, a_{i} \in A, b_{i} \in B, i=1,2 .
$$

Proposition 8. If the $\varepsilon_{0}$-graded multiplication (66) satisfies (cf. (4))

$$
\varepsilon_{0}\left(b_{1}^{\prime}, a_{2}^{\prime}\right)\left(a_{1} \otimes b_{1}\right) \star^{\left(\varepsilon_{0}\right)}\left(a_{2} \otimes b_{2}\right)=\left(a_{1} \cdot a a_{2}\right) \otimes\left(b_{1} \cdot b b_{2}\right),
$$

then $\mathscr{A}_{2}^{\left(\varepsilon_{0}\right)} \otimes \mathscr{B}_{2}^{\left(\varepsilon_{0}\right)}$ is a $\varepsilon_{0}$-graded commutative algebra.

Proposition 9. If $\mathscr{A}_{2}^{\left(\varepsilon_{0}\right)}$ and $\mathscr{B}_{2}^{\left(\varepsilon_{0}\right)}$ are associative, then $\mathscr{A}_{2}^{\left(\varepsilon_{0}\right)} \otimes \mathscr{B}_{2}^{\left(\varepsilon_{0}\right)}$ is also associative.

Proof. This follows from (66), (67) and the properties of the commutation factor $\varepsilon_{0}(6)-(7)$.

In the matrix form (67) becomes (with $\star^{\left(\varepsilon_{0}\right)} \equiv \mu_{2}^{\star\left(\varepsilon_{0}\right)}$ )

$$
\varepsilon_{0}\left(b_{1}^{\prime}, a_{2}^{\prime}\right) \mu_{2}^{\star\left(\varepsilon_{0}\right)}\left[\begin{array}{l}
\mu_{2}^{\otimes}\left[a_{1}, b_{1}\right] \\
\mu_{2}^{\otimes}\left[a_{2}, b_{2}\right]
\end{array}\right]=\mu_{2}^{\otimes}\left[\begin{array}{l}
\mu_{2}^{(a)}\left[a_{1}, a_{2}\right] \\
\mu_{2}^{(b)}\left[b_{1}, b_{2}\right]
\end{array}\right],
$$

where $\mu_{2}^{\otimes}$ is the standard binary tensor product. For numerous generalizations (including braidings), see, e.g., [40], and references therein.

Now we can extend (68) to almost medial algebras. 
Definition 24. Let $\mathscr{A}_{2}^{\left(\rho_{0}\right)}$ and $\mathscr{B}_{2}^{\left(\rho_{0}\right)}$ be two binary medial algebras with the same mediality factor $\rho_{0}$. The total $\rho_{0}$-mediality graded multiplication $\mu_{2}^{\star\left(\rho_{0}\right)}:\left(\mathscr{A}_{2}^{\left(\rho_{0}\right)} \otimes \mathscr{B}_{2}^{\left(\rho_{0}\right)}\right) \star{ }^{\left(\rho_{0}\right)}$ $\left(\mathscr{A}_{2}^{\left(\rho_{0}\right)} \otimes \mathscr{B}_{2}^{\left(\rho_{0}\right)}\right) \longrightarrow \mathscr{A}_{2}^{\left(\rho_{0}\right)} \otimes \mathscr{B}_{2}^{\left(\rho_{0}\right)}$ is defined by the mediality deformation (cf. (2))

$$
\rho_{0}\left(\begin{array}{ll}
a_{1}^{\prime} & b_{1}^{\prime} \\
a_{2}^{\prime} & b_{2}^{\prime}
\end{array}\right)\left(a_{1} \otimes b_{1}\right) \star^{\left(\rho_{0}\right)}\left(a_{2} \otimes b_{2}\right), b_{1}^{\prime}, a_{2}^{\prime} \in G, a_{i} \in A, b_{i} \in B, i=1,2 .
$$

Proposition 10. If the $\rho_{0}$-graded multiplication (69) satisfies (cf. (4))

$$
\rho_{0}\left(\begin{array}{ll}
a_{1}^{\prime} & b_{1}^{\prime} \\
a_{2}^{\prime} & b_{2}^{\prime}
\end{array}\right) \mu_{2}^{\star\left(\rho_{0}\right)}\left[\begin{array}{l}
\mu_{2}^{\otimes}\left[a_{1}, b_{1}\right] \\
\mu_{2}^{\otimes}\left[a_{2}, b_{2}\right]
\end{array}\right]=\mu_{2}^{\otimes}\left[\begin{array}{l}
\mu_{2}^{(a)}\left[a_{1}, a_{2}\right] \\
\mu_{2}^{(b)}\left[b_{1}, b_{2}\right]
\end{array}\right],
$$

then $\mathscr{A}_{2}^{\left(\rho_{0}\right)} \otimes \mathscr{B}_{2}^{\left(\rho_{0}\right)}$ is a $\rho_{0}$-graded binary (almost medial) algebra.

Using the matrix form (70) one can generalize the $\rho_{0}$-graded medial algebras to arbitrary arity.

Let $\mathscr{B}_{n}^{\left(\rho_{0}\right), 1}, \ldots, \mathscr{B}_{n}^{\left(\rho_{0}\right), n}$ be $n \rho_{0}$-graded (almost medial) $n$-ary algebras $\left(\mathscr{B}_{n}^{\left(\rho_{0}\right), i}=\right.$ $\left\langle B_{i} \mid \mu_{n}^{(i)}\right\rangle$ ) with the same mediality factor $\rho_{0}$ and the same graded structure. Consider their tensor product $\mathscr{B}_{n}^{\left(\rho_{0}\right), 1} \otimes \ldots \otimes \mathscr{B}_{n}^{\left(\rho_{0}\right), n}$ and the $\rho_{0}$-graded $n$-ary multiplication $\mu_{n}^{\star\left(\rho_{0}\right)}$ on it.

Proposition 11. If the $\rho_{0}$-graded n-ary multiplication $\mu_{n}^{\star\left(\rho_{0}\right)}$ satisfies (cf. (4))

$$
\begin{aligned}
& \rho_{0}\left(\begin{array}{ccc}
b_{1}^{1 \prime} & \ldots & b_{1}^{n \prime} \\
\vdots & \ldots & \vdots \\
b_{n}^{1 \prime} & \ldots & b_{n}^{n \prime}
\end{array}\right) \mu_{n}^{\star\left(\rho_{0}\right)}\left[\begin{array}{c}
\mu_{n}^{\otimes}\left[b_{1}^{1}, \ldots, b_{1}^{n}\right] \\
\vdots \\
\mu_{n}^{\otimes}\left[b_{n}^{1}, \ldots, b_{n}^{n}\right]
\end{array}\right]=\mu_{n}^{\otimes}\left[\begin{array}{c}
\mu_{n}^{(1)}\left[b_{1}^{1}, \ldots, b_{n}^{1}\right] \\
\vdots \\
\mu_{n}^{(n)}\left[b_{n}^{1}, \ldots, b_{n}^{n}\right]
\end{array}\right], \\
& b_{1}^{i}, \ldots, b_{n}^{i} \in \mathscr{A}_{n}^{\left(\rho_{0}\right), i}, b_{n}^{i \prime}, \ldots, b_{n}^{i \prime} \in G, i=1, \ldots, n .
\end{aligned}
$$

then the tensor product $\mathscr{B}_{n}^{\left(\rho_{0}\right), 1} \otimes \ldots \otimes \mathscr{B}_{n}^{\left(\rho_{0}\right), n}$ is a $\rho_{0}$-graded $n$-ary (almost medial) algebra.

Symbolically, we can write this in the form, similar to the almost mediality condition (63)

$$
\boldsymbol{\rho}_{0}\left(\hat{\boldsymbol{B}}_{\left(n^{2}\right)}^{\prime}\right) \mu_{n}^{\star\left(\rho_{0}\right)} \circ \mu_{n^{2}}^{\otimes}\left[\hat{\boldsymbol{B}}_{\left(n^{2}\right)}\right]=\mu_{n}^{\otimes} \circ \mu_{n}^{(1)} \circ \ldots \circ \mu_{n}^{(n)}\left[\hat{\boldsymbol{B}}_{\left(n^{2}\right)}^{T}\right],
$$

where

$$
\hat{\boldsymbol{B}}_{\left(n^{2}\right)}^{\prime}=\left(\begin{array}{ccc}
b_{1}^{1 \prime} & \ldots & b_{1}^{n \prime} \\
\vdots & \ldots & \vdots \\
b_{n}^{1 \prime} & \ldots & b_{n}^{n \prime}
\end{array}\right), \quad \hat{\boldsymbol{B}}_{\left(n^{2}\right)}=\left(\begin{array}{ccc}
b_{1}^{1} & \ldots & b_{1}^{n} \\
\vdots & \ldots & \vdots \\
b_{n}^{1} & \ldots & b_{n}^{n}
\end{array}\right)
$$

and $\hat{\boldsymbol{B}}_{\left(n^{2}\right)}^{T}$ is its transpose.

Example 3. In the lowest nonbinary example, for three ternary $\rho_{0}$-graded algebras $\mathscr{A}_{3}^{\left(\rho_{0}\right)}=$ $\left\langle A \mid \mu_{3}^{(a)}\right\rangle, \mathscr{B}_{3}^{\left(\rho_{0}\right)}=\left\langle B \mid \mu_{3}^{(b)}\right\rangle, \mathscr{C}_{3}^{\left(\rho_{0}\right)}=\left\langle C \mid \mu_{3}^{(c)}\right\rangle$, from (71) we have the ternary multiplication $\mu_{3}^{\star\left(\rho_{0}\right)}$ for their ternary tensor product $\mathscr{A}_{3}^{\left(\rho_{0}\right)} \otimes \mathscr{B}_{3}^{\left(\rho_{0}\right)} \otimes \mathscr{C}_{3}^{\left(\rho_{0}\right)}$ given by

$$
\begin{aligned}
& \boldsymbol{\rho}_{0}\left(\begin{array}{lll}
a_{1}^{\prime} & b_{1}^{\prime} & c_{1}^{\prime} \\
a_{2}^{\prime} & b_{2}^{\prime} & c_{2}^{\prime} \\
a_{3}^{\prime} & b_{3}^{\prime} & c_{3}^{\prime}
\end{array}\right) \mu_{3}^{\star\left(\rho_{0}\right)}\left(\begin{array}{c}
\left(a_{1} \otimes b_{1} \otimes c_{1}\right) \\
\left(a_{2} \otimes b_{2} \otimes c_{2}\right) \\
\left(a_{3} \otimes b_{2} \otimes c_{3}\right)
\end{array}\right) \\
& =\left(\mu_{3}^{(a)}\left[a_{1}, a_{2}, a_{3}\right] \otimes \mu_{3}^{(a)}\left[b_{1}, b_{2}, b_{3}\right] \otimes \mu_{3}^{(c)}\left[c_{1}, c_{2}, c_{3}\right]\right),
\end{aligned}
$$


where $a_{i} \in A, b_{i} \in B, c_{i} \in C, a_{i}^{\prime}, b_{i}^{\prime}, c_{i}^{\prime} \in G, i=1,2,3$.

Higher Level Mediality $n^{2}$-Ary Brackets

Binary almost mediality algebras for $n=2$ were considered in (35), together with the tower of mediality factors (45), (48)-(50). Here we generalize this construction to any arity $n$ which can be done using the matrix polyad construction.

First, we deform the almost mediality condition (63)

$$
\rho_{0}^{\left(n^{2}\right)}\left(\hat{A}_{\left(n^{2}\right)}^{\prime}\right) \mathrm{A}_{n^{2}}^{(\mu)}=\mathrm{A}_{n^{2}}^{T(\mu)}+\mathrm{M}_{0}^{\left(\rho_{0}\right)}\left(\hat{A}_{\left(n^{2}\right)}\right), \quad a_{i j} \in A ; a_{i j}^{\prime} \in G, i, j=1, \ldots, n,
$$

where $\mathrm{M}_{0}^{\left(\rho_{0}\right)}: A^{\otimes n^{2}} \rightarrow A$ is the higher mediality $n^{2}$-ary bracket of 0-level. Consider $\mathrm{M}_{0}^{\left(\rho_{0}\right)}$ as a new $n^{2}$-ary (bracket) multiplication

$$
\mu_{n^{2}}^{\left(\rho_{0}, M_{0}\right)}\left[\hat{A}_{\left(n^{2}\right)}\right]:=\mathrm{M}_{0}^{\left(\rho_{0}\right)}\left(\hat{A}_{\left(n^{2}\right)}\right) .
$$

Definition 25. $A n^{2}$-ary algebra

$$
\mathscr{A}_{n^{2}}^{\left(\rho_{0}, M_{0}\right)}=\left\langle A \mid \boldsymbol{\mu}_{n^{2}}^{\left(\rho_{0}, M_{0}\right)}\right\rangle
$$

is called a 0-level mediality bracket $n^{2}$-ary algebra.

Proposition 12. The $n^{2}$-ary algebra $\mathscr{A}_{n^{2}}^{\left(\rho_{0}, M_{0}\right)}$ is almost medial with the mediality factor $\left(-\left(\rho_{0}^{\left(n^{2}\right)}\right)^{-1}\right)$.

Proof. We multiply the definition (76) by $\rho_{0}^{\left(n^{2}\right)}\left(\left(\hat{A}_{\left(n^{2}\right)}^{\prime}\right)^{T}\right)$ and use (65) to obtain

$$
\boldsymbol{\rho}_{0}^{\left(n^{2}\right)}\left(\left(\hat{A}_{\left(n^{2}\right)}^{\prime}\right)^{T}\right) \mathrm{M}_{0}^{\left(\rho_{0}\right)}\left(\hat{A}_{\left(n^{2}\right)}\right)=\mathrm{A}_{n^{2}}^{(\mu)}-\boldsymbol{\rho}_{0}^{\left(n^{2}\right)}\left(\left(\hat{A}_{\left(n^{2}\right)}^{\prime}\right)^{T}\right) \mathrm{A}_{n^{2}}^{T(\mu)}
$$

Taking into account that the r.h.s. here is exactly $-\mathrm{M}_{0}^{\left(\rho_{0}\right)}\left(\hat{A}_{\left(n^{2}\right)}^{T}\right)$, we have

$$
-\boldsymbol{\rho}_{0}^{\left(n^{2}\right)}\left(\left(\hat{A}_{\left(n^{2}\right)}^{\prime}\right)^{T}\right) \mathrm{M}_{0}^{\left(\rho_{0}\right)}\left(\hat{A}_{\left(n^{2}\right)}\right)=\mathrm{M}_{0}^{\left(\rho_{0}\right)}\left(\hat{A}_{\left(n^{2}\right)}^{T}\right),
$$

and using (65) again, we get

$$
-\rho_{0}^{\left(n^{2}\right)}\left(\hat{A}_{\left(n^{2}\right)}^{\prime}\right)^{-1} \mathrm{M}_{0}^{\left(\rho_{0}\right)}\left(\hat{A}_{\left(n^{2}\right)}\right)=\mathrm{M}_{0}^{\left(\rho_{0}\right)}\left(\hat{A}_{\left(n^{2}\right)}^{T}\right),
$$

which should be compared with (63).

Now we "deform" (76) successively by defining further $n^{2}$-ary brackets $\mathrm{M}_{k}$ and higher level mediality factors $\boldsymbol{\rho}_{k}^{\left(n^{2}\right)}: G^{\times n^{2}} \rightarrow \mathbb{k}$ as follows. 
Definition 26. The k-level mediality $n^{2}$-ary brackets and factors are defined by

$$
\begin{aligned}
\rho_{1}^{\left(n^{2}\right)}\left(\hat{A}_{\left(n^{2}\right)}^{\prime}\right) \mathrm{M}_{0}^{\left(\rho_{0}\right)}\left(\hat{A}_{\left(n^{2}\right)}\right) & =\mathrm{M}_{0}^{\left(\rho_{0}\right)}\left(\hat{A}_{\left(n^{2}\right)}^{T}\right)+\mathrm{M}_{1}^{\left(\rho_{0}, \rho_{1}\right)}\left(\hat{A}_{\left(n^{2}\right)}\right), \\
\rho_{2}^{\left(n^{2}\right)}\left(\hat{A}_{\left(n^{2}\right)}^{\prime}\right) \mathrm{M}_{1}^{\left(\rho_{0}, \rho_{1}\right)}\left(\hat{A}_{\left(n^{2}\right)}\right) & =\mathrm{M}_{1}^{\left(\rho_{0}, \rho_{1}\right)}\left(\hat{A}_{\left(n^{2}\right)}^{T}\right)+\mathrm{M}_{2}^{\left(\rho_{0}, \rho_{1}, \rho_{2}\right)}\left(\hat{A}_{\left(n^{2}\right)}\right), \\
\vdots & \\
\boldsymbol{\rho}_{k}^{\left(n^{2}\right)}\left(\hat{A}_{\left(n^{2}\right)}^{\prime}\right) \mathrm{M}_{k-1}^{\left(\rho_{0}, \rho_{1}, \ldots, \rho_{k-1}\right)}\left(\hat{A}_{\left(n^{2}\right)}\right) & =\mathrm{M}_{k-1}^{\left(\rho_{0}, \rho_{1}, \ldots, \rho_{k-1}\right)}\left(\hat{A}_{\left(n^{2}\right)}^{T}\right)+\mathrm{M}_{k}^{\left(\rho_{0}, \rho_{1}, \ldots, \rho_{k}\right)}\left(\hat{A}_{\left(n^{2}\right)}\right) \\
\forall a_{i j} & \in A ; a_{i j}^{\prime} \in G, i, j=1, \ldots, n .
\end{aligned}
$$

Definition 27. $k$-level $n^{2}$-ary almost mediality is given by the vanishing of the last "deforming" medial $n^{2}$-ary bracket

$$
\mathrm{M}_{k}^{\left(\rho_{0}, \rho_{1}, \ldots, \rho_{k}\right)}\left(\hat{A}_{\left(n^{2}\right)}\right)=0, \quad \forall a_{i j} \in A,
$$

and has the form

$$
\rho_{k}^{\left(n^{2}\right)}\left(\hat{A}_{\left(n^{2}\right)}^{\prime}\right) \mathrm{M}_{k-1}^{\left(\rho_{0}, \rho_{1}, \ldots, \rho_{k-1}\right)}\left(\hat{A}_{\left(n^{2}\right)}\right)=\mathrm{M}_{k-1}^{\left(\rho_{0}, \rho_{1}, \ldots, \rho_{k-1}\right)}\left(\hat{A}_{\left(n^{2}\right)}^{T}\right) .
$$

Proposition 13. The higher level "deforming" functions ( $n^{2}$-ary brackets) $\mathrm{M}_{i}^{\left(\rho_{0}, \rho_{1}, \ldots, \rho_{k}\right)}\left(\hat{A}_{\left(n^{2}\right)}\right)$, $i=1, \ldots, k$ can be expressed through $\mathrm{M}_{0}^{\left(\rho_{0}\right)}\left(\hat{A}_{\left(n^{2}\right)}\right)$ from (76) using a combination of the lower level $n^{2}$-ary mediality factors $\rho_{k}^{\left(n^{2}\right)}\left(\hat{A}_{\left(n^{2}\right)}^{\prime}\right), i=1, \ldots, k$.

Proof. This follows from Equations (82)-(84).

\section{Toyoda's Theorem for Almost Medial Algebras}

The structure of the almost medial graded algebras (binary and $n$-ary) can be established by searching for possible analogs of Toyoda's theorem (28) (see, $[8,29,30])$ which is the main statement for medial groupoids [23] and quasigroups [28]. As Toyoda's theorem connects medial algebras with abelian algebras, we can foresee that in the same way the almost medial algebras can be connected with almost commutative algebras.

First, let us consider almost medial graded binary algebras, as defined in (35)-(39).

Theorem 2. Let $\mathscr{A}_{2}^{(\rho)}=\left\langle A \mid \mu_{2}\right\rangle$ be an almost medial ( $\rho$-commutative) G-graded binary algebra, then there exists an almost commutative (e-commutative G-graded binary algebra $\overline{\mathscr{A}}_{2}^{(\varepsilon)}=\left\langle A \mid \bar{\mu}_{2}\right\rangle$, two grading preserving automorphisms $\varphi_{1,2}$ and a fixed element $h \in A$, such that (cf. (28))

$$
\begin{aligned}
\mu_{2}[a, b] & =\bar{\mu}_{2}\left[\bar{\mu}_{2}\left[\varphi_{1}(a), \varphi_{2}(b)\right], h\right] \text { or } a \cdot b=\varphi_{1}(a) \varphi_{2}(b) h, \\
\rho\left(a^{\prime}, b^{\prime}, c^{\prime}, d^{\prime}\right) & =\varepsilon\left(b^{\prime}, c^{\prime}\right), \forall a, b, c, d \in A, \quad a^{\prime}, b^{\prime}, c^{\prime}, d^{\prime} \in G
\end{aligned}
$$

where we denote $\mu_{2} \equiv(\cdot)$ and $\bar{\mu}_{2}[a, b] \equiv a b$. We use the multiplicative notation for the algebra $\overline{\mathscr{A}}_{2}^{(\varepsilon)}$, because it is non-commutative. 
Proof. We use the "linear" presentation (87) for the product in $\mathscr{A}_{2}^{(\rho)}$ and insert it into the condition of almost mediality (35) to obtain

$$
\begin{aligned}
& \rho\left(a^{\prime}, b^{\prime}, c^{\prime}, d^{\prime}\right)(a \cdot b) \cdot(c \cdot d)=(a \cdot c) \cdot(b \cdot d) \Rightarrow \\
& \rho\left(a^{\prime}, b^{\prime}, c^{\prime}, d^{\prime}\right) \varphi_{1}\left(\varphi_{1}(a) \varphi_{2}(b) h\right) \varphi_{2}\left(\left(\varphi_{1}(c) \varphi_{2}(d) h\right)\right) h \\
& =\varphi_{1}\left(\varphi_{1}(a) \varphi_{2}(c) h\right) \varphi_{2}\left(\left(\varphi_{1}(b) \varphi_{2}(d) h\right)\right) \Rightarrow \\
& \rho\left(a^{\prime}, b^{\prime}, c^{\prime}, d^{\prime}\right) \varphi_{1} \circ \varphi_{1}(a) \varphi_{1} \circ \varphi_{2}(b) \varphi_{1}(h) \varphi_{2} \circ \varphi_{1}(c) \varphi_{2} \circ \varphi_{2}(d) \varphi_{2}(h) h \\
& =\varphi_{1} \circ \varphi_{1}(a) \varphi_{1} \circ \varphi_{2}(c) \varphi_{1}(h) \varphi_{2} \circ \varphi_{1}(b) \varphi_{2} \circ \varphi_{2}(d) \varphi_{2}(h) h,
\end{aligned}
$$

where (०) is the composition of automorphisms. Using the cancellativity of $\overline{\mathscr{A}}_{2}^{(\varepsilon)}$, we get

$$
\rho\left(a^{\prime}, b^{\prime}, c^{\prime}, d^{\prime}\right) \varphi_{1} \circ \varphi_{2}(b) \varphi_{2} \circ \varphi_{1}(c)=\varphi_{1} \circ \varphi_{2}(c) \varphi_{2} \circ \varphi_{1}(b) .
$$

Because the automorphisms $\varphi_{1,2}$ preserve grading, after implementing almost $(\varepsilon-)$ commutativity (4), the r.h.s. of (90) becomes $\varepsilon\left(b^{\prime}, c^{\prime}\right) \varphi_{2} \circ \varphi_{1}(b) \varphi_{1} \circ \varphi_{2}(c)$ which gives (88) for commuting automorphisms.

The higher arity cases are more nontrivial and very cumbersome. Therefore, we restrict ourselves by the case $n=3$ only.

Theorem 3. Let $\mathscr{A}_{3}^{(\rho)}=\left\langle A \mid \mu_{3}, v_{2}\right\rangle$ be an almost medial ( $\rho$-commutative) G-graded ternary algebra over a field $\mathbb{k}$. Then there exists an almost commutative ( $\varepsilon$-commutative $G$-graded binary algebra $\overline{\mathscr{A}}_{2}^{(\varepsilon)}=\left\langle A \mid \bar{\mu}_{2}\right\rangle$, three commuting grading preserving automorphisms $\varphi_{1,2,3}$ and a fixed element $h \in A$, such that (cf. (28))

$$
\begin{aligned}
\mu_{3}[a, b, c] & =\bar{\mu}_{2}\left[\bar{\mu}_{2}\left[\bar{\mu}_{2}\left[\varphi_{1}(a), \varphi_{2}(b)\right], h\right], h\right] \\
& \equiv \varphi_{1}(a) \varphi_{2}(b) \varphi_{3}(c) h, \forall a, b, c, h \in A \\
\rho^{\left(3^{2}\right)}\left(\hat{A}_{\left(3^{2}\right)}^{\prime}\right) & =\varepsilon\left(a_{12}^{\prime}, a_{31}^{\prime}\right) \varepsilon\left(a_{12}^{\prime}, a_{21}^{\prime}\right) \varepsilon\left(a_{13}^{\prime}, a_{31}^{\prime}\right) \varepsilon\left(a_{13}^{\prime}, a_{32}^{\prime}\right) \varepsilon\left(a_{23}^{\prime}, a_{32}^{\prime}\right) \varepsilon\left(a_{23}^{\prime}, a_{31}^{\prime}\right), \\
\hat{A}_{\left(3^{2}\right)}^{\prime} & =\left(a_{i j}^{\prime}\right), \quad \forall a_{i j}^{\prime} \in G, \quad i, j=1, \ldots, 3,
\end{aligned}
$$

where we denote $\bar{\mu}_{2}[a, b] \equiv a b$.

Proof. Using the matrix form of ternary $(n=3)$ almost regularity (63) and inserting there the ternary "linear" presentation (91) we get (in matrix form), $\forall a_{i j} \in A, i, j=1, \ldots, 3$,

$$
\begin{aligned}
\rho^{\left(3^{2}\right)}\left(\hat{A}_{\left(3^{2}\right)}^{\prime}\right) & \left(\begin{array}{lll}
\varphi_{1} \circ \varphi_{1}\left(a_{11}\right) & \varphi_{1} \circ \varphi_{2}\left(a_{12}\right) & \varphi_{1} \circ \varphi_{3}\left(a_{13}\right) \\
\varphi_{2} \circ \varphi_{1}\left(a_{21}\right) & \varphi_{2} \circ \varphi_{2}\left(a_{22}\right) & \varphi_{2} \circ \varphi_{3}\left(a_{23}\right) \\
\varphi_{3} \circ \varphi_{1}\left(a_{31}\right) & \varphi_{3} \circ \varphi_{2}\left(a_{32}\right) & \varphi_{3} \circ \varphi_{3}\left(a_{33}\right)
\end{array}\right) \\
= & \left(\begin{array}{lll}
\varphi_{1} \circ \varphi_{1}\left(a_{11}\right) & \varphi_{1} \circ \varphi_{2}\left(a_{21}\right) & \varphi_{1} \circ \varphi_{3}\left(a_{31}\right) \\
\varphi_{2} \circ \varphi_{1}\left(a_{12}\right) & \varphi_{2} \circ \varphi_{2}\left(a_{22}\right) & \varphi_{2} \circ \varphi_{3}\left(a_{32}\right) \\
\varphi_{3} \circ \varphi_{1}\left(a_{13}\right) & \varphi_{3} \circ \varphi_{2}\left(a_{23}\right) & \varphi_{3} \circ \varphi_{3}\left(a_{33}\right)
\end{array}\right) .
\end{aligned}
$$

Applying the cancellativity of the binary algebra $\overline{\mathscr{A}}_{2}^{(\varepsilon)}$, we have

$$
\begin{aligned}
& \boldsymbol{\rho}^{\left(3^{2}\right)}\left(\hat{A}_{\left(3^{2}\right)}^{\prime}\right) \varphi_{1} \circ \varphi_{2}\left(a_{12}\right) \varphi_{1} \circ \varphi_{3}\left(a_{13}\right) \varphi_{2} \circ \varphi_{1}\left(a_{21}\right) \varphi_{2} \circ \varphi_{3}\left(a_{23}\right) \varphi_{3} \circ \varphi_{1}\left(a_{31}\right) \varphi_{3} \circ \varphi_{2}\left(a_{32}\right) \\
& =\varphi_{1} \circ \varphi_{2}\left(a_{21}\right) \varphi_{1} \circ \varphi_{3}\left(a_{31}\right) \varphi_{2} \circ \varphi_{1}\left(a_{12}\right) \varphi_{2} \circ \varphi_{3}\left(a_{32}\right) \varphi_{3} \circ \varphi_{1}\left(a_{13}\right) \varphi_{3} \circ \varphi_{2}\left(a_{23}\right) .
\end{aligned}
$$

Implementing almost ( $\varepsilon$-) commutativity (4) on the r.h.s. of (95), we arrive (for pairwise commuting grading preserving automorphisms $\left.\varphi_{i} \circ \varphi_{j}=\varphi_{j} \circ \varphi_{i}, i, j=1,2,3\right)$ at (92). 


\section{Binary Tensor Categories}

We now apply the above ideas to construct a special kind of categories with multiplication [41,42] which appeared already in [43] and later on they were called tensor categories and monoidal categories (as they "remind" us of the structure of a monoid) [13]. For reviews, see, e.g., [44,45]. The monoidal categories can be considered as the categorification [46] of a monoid object and can be treated as an instance of the microcosm principle: "certain algebraic structures can be defined in any category equipped with a categorified version of the same structure" [47]. We start from the definitions of categories [48,49] and binary tensor categories [13] (in our notation).

Let $\mathscr{C}$ be a category with the class of objects $\mathrm{Ob} \mathscr{C}$ and morphisms Mor $\mathscr{C}$, such that the arrow from the source $X_{1}$ to the target $X_{2}$ is defined by Mor $\mathscr{C} \ni f_{12}: X_{1} \rightarrow X_{2}$, $X_{1,2} \in \mathrm{Ob} \mathscr{C}$, and usually $\operatorname{Hom}_{C}\left(X_{1}, X_{2}\right)$ denotes all arrows which do not intersect. If $\mathrm{Ob} \mathscr{C}$ and Mor $\mathscr{C}$ are sets, the category is small. The composition (o) of three morphisms, their associativity and the identity morphism $\left(\mathrm{id}_{X}\right)$ are defined in the standard way [13].

If $\mathscr{C}$ and $\mathscr{C}^{\prime}$ are two categories, then a mapping between them is called a covariant functor $\mathcal{F}: \mathscr{C} \rightarrow \mathscr{C}^{\prime}$ which consists of two different components: (1) the X-component is a mapping of objects $\mathcal{F}_{\mathrm{Ob}}: \mathrm{Ob} \mathscr{C} \rightarrow \mathrm{Ob} \mathscr{C}^{\prime}$; (2) the $\mathrm{f}$-component is a mapping of morphisms $\mathcal{F}_{\text {Mor }}:$ Mor $\mathscr{C} \rightarrow$ Mor $\mathscr{C}^{\prime}$ such that $\mathcal{F}=\left\{\mathcal{F}_{\mathrm{Ob}}, \mathcal{F}_{\text {Mor }}\right\}$. A functor preserves the identity morphism $\mathcal{F}_{\text {Mor }}\left(\mathrm{id}_{X}\right)=\mathrm{id}_{\mathcal{F}_{\mathrm{Ob}}(X)}$ and the composition of morphisms $\mathcal{F}_{\mathrm{Mor}}\left(\mathrm{f}_{23} \circ \mathrm{f}_{12}\right)=$ $\mathcal{F}_{\text {Mor }}\left(f_{23}\right) \circ^{\prime} \mathcal{F}_{\text {Mor }}\left(f_{12}\right)\left(=\mathcal{F}_{\text {Mor }}\left(f_{12}\right) \circ^{\prime} \mathcal{F}_{\text {Mor }}\left(f_{23}\right)\right.$ for a contravariant functor $)$, where $\left(\circ^{\prime}\right)$ is the composition in $\mathscr{C}^{\prime}$.

The (binary) product category $\mathscr{C} \times \mathscr{C}^{\prime}$ consists of all pairs of objects $\left(\mathrm{Ob} \mathscr{C}, \mathrm{Ob} \mathscr{C}^{\prime}\right)$, morphisms (Mor $\mathscr{C}, \operatorname{Mor} \mathscr{C}^{\prime}$ ) and identities $\left(\mathrm{id}_{X}, \mathrm{id}_{X^{\prime}}\right)$, while the composition $\left(0^{\prime \prime}\right)$ is made component-wise

$$
\begin{aligned}
& \left(\mathrm{f}_{23}, \mathrm{f}_{23}^{\prime}\right) \circ^{\prime \prime}\left(\mathrm{f}_{12}, \mathrm{f}_{12}^{\prime}\right)=\left(\mathrm{f}_{23} \circ \mathrm{f}_{12}, \mathrm{f}_{23}^{\prime} \circ \mathrm{o}_{12}^{\prime}\right), \\
& \mathrm{f}_{i j}: X_{i} \rightarrow X_{j}, \forall X_{i} \in \mathrm{Ob} \mathscr{C}, \mathrm{f}_{i j}^{\prime}: X_{i}^{\prime} \rightarrow X_{j}^{\prime}, \forall X_{i}^{\prime} \in \mathrm{Ob} \mathscr{C}^{\prime}, i, j=1,2,3,
\end{aligned}
$$

and by analogy this may be extended for more multipliers. A functor on a binary product category is called a bifunctor (multifunctor). A functor consists of two components $\left\{\mathcal{F}_{\mathrm{Ob}}, \mathcal{F}_{\mathrm{Mor}}\right\}$, and therefore a mapping between two functors $\mathcal{F}$ and $\mathcal{G}$ should also be two-component $\mathbf{T}^{F G}=\left\{\mathrm{T}_{\mathrm{Ob}}^{F G}, \mathbf{T}_{\mathrm{Mor}}^{F G}\right\}$. Usually [13], the components are denoted by the same letter, but for clarity we will distinguish them, because their action, arguments and corresponding commutative diagrams are different. Without other conditions $\mathbf{T}^{F G}$ is called an infranatural transformation from $\mathcal{F}$ to $\mathcal{G}$. A natural transformation (denoted by the double arrow $\mathrm{T}^{F G}: \mathcal{F} \Rightarrow \mathcal{G}$ ) is defined by the consistency condition of the above mappings in $\mathscr{C}^{\prime}$

$$
\mathrm{T}_{\mathrm{Ob}}^{F G} \circ^{\prime} \mathcal{F}_{\mathrm{Mor}}=\mathcal{G}_{\mathrm{Mor}} \circ^{\prime} \mathrm{T}_{\mathrm{Ob}}^{F G} .
$$

Application to objects gives the following commutative diagram for the natural transformations (bifunctoriality)

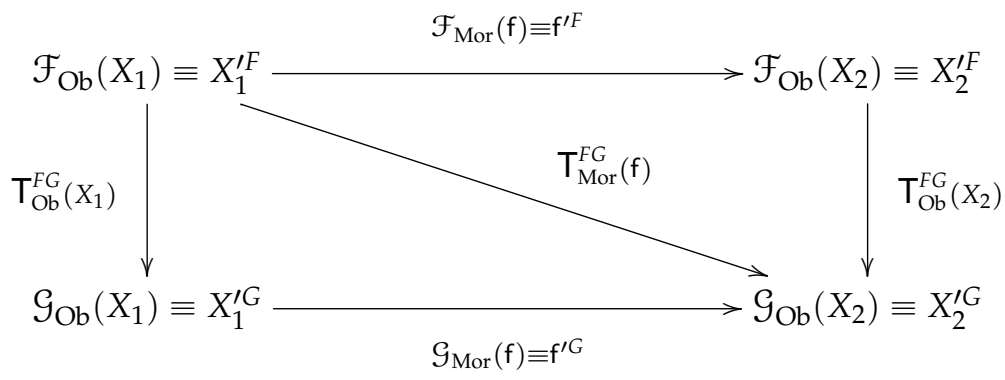


which is the consistency of the objects in $\mathscr{C}^{\prime}$ transformed by $\mathcal{F}$ and $\mathcal{G}$. The the diagonal in (98) may also be interpreted as the action of the natural transformation on a morphism $\mathrm{T}_{\text {Mor }}^{F G}(\mathrm{f}): \mathcal{F}_{\mathrm{Ob}}\left(X_{1}\right) \rightarrow \mathcal{G}_{\mathrm{Ob}}\left(X_{2}\right), \mathrm{f}: X_{1} \rightarrow X_{2}, \mathrm{f} \in \operatorname{Mor} \mathscr{C}, X_{1}, X_{2} \in \mathrm{Ob} \mathscr{C}$, such that

$$
\mathbf{T}_{\text {Mor }}^{F G}(\mathbf{f})=\mathbf{T}_{\mathrm{Ob}}^{F G}\left(X_{2}\right) \circ^{\prime} \mathcal{F}_{\text {Mor }}(\mathrm{f})=\mathcal{G}_{\mathrm{Mor}}(\mathrm{f}) \circ^{\prime} \mathbf{T}_{\mathrm{Ob}}^{F G}\left(X_{1}\right),
$$

where the second equality holds valid due to the naturality (97).

In a concise form the natural transformations are described by the commutative diagram

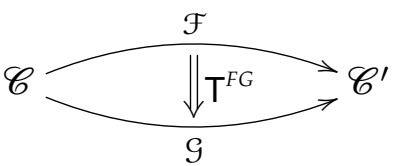

For a category $\mathscr{C}$, the identity functor $\mathcal{J} d_{\mathscr{C}}=\left(\mathcal{J} d_{\mathscr{C}, \mathrm{Ob}}, \mathcal{J} d_{\mathscr{C}, \text { Mor }}\right)$ is defined by $\mathcal{J} d_{\mathscr{C}, \mathrm{Ob}}(X)=X, \mathcal{J}_{\mathscr{C}, \operatorname{Mor}}(\mathrm{f})=\mathrm{f}, \forall X \in \mathrm{Ob} \mathscr{C}, \forall \mathrm{f} \in \operatorname{Mor} \mathscr{C}$. Two categories $\mathscr{C}$ and $\mathscr{C}^{\prime}$ are equivalent, if there exist two functors $\mathcal{F}$ and $\mathcal{G}$ and two natural transformations $\mathrm{T}^{F G}: \mathcal{J} d_{\mathscr{C}^{\prime}} \Rightarrow \mathcal{F} \circ^{\prime} \mathcal{G}$ and $\mathrm{T}^{G F}: \mathcal{G} \circ \mathcal{F} \Rightarrow \mathcal{J} d_{\mathscr{C}}$.

For more details and standard properties of categories, see, e.g., $[13,48,49]$ and references therein.

The categorification $[46,50]$ of most algebraic structures can be provided by endowing categories with an additional operation [41,42] "reminding" us of the tensor product [13].

A binary "magmatic" tensor category is $\left(\mathscr{C}, \mathcal{M}^{(2 \otimes)}\right)$, where $\mathcal{M}^{(2 \otimes)} \equiv \otimes: \mathscr{C} \times \mathscr{C} \rightarrow \mathscr{C}$ is a bifunctor. We use this notation with brackets $\mathcal{M}^{(2 \otimes)}$ [51], because they are convenient for further consideration of the $n$-ary case [20]. In component form the bifunctor is $\mathcal{M}^{(2 \otimes)}=$ $\left\{\mathcal{M}_{\mathrm{Ob}}^{(2 \otimes)}, \mathcal{M}_{\mathrm{Mor}}^{(2 \otimes)}\right\}$, where $\mathcal{M}_{\mathrm{Mor}}^{(2 \otimes)}$ is

$$
\begin{aligned}
& \mathcal{M}_{\mathrm{Mor}}^{(2 \otimes)}\left[\mathrm{f}_{11^{\prime}}, \mathrm{f}_{22^{\prime}}\right]=\mathcal{M}_{\mathrm{Ob}}^{(2 \otimes)}\left[X_{1}, X_{2}\right] \rightarrow \mathcal{M}_{\mathrm{Ob}}^{(2 \otimes)}\left[X_{1}^{\prime}, X_{2}^{\prime}\right], \\
& \mathrm{f}_{i i^{\prime}}: X_{i} \rightarrow X_{i}^{\prime}, \mathrm{f}_{i i^{\prime}} \in \operatorname{Mor} \mathscr{C}, \forall X_{i}, X_{i^{\prime}} \in \mathrm{Ob} \mathscr{C}, \quad i=1,2 .
\end{aligned}
$$

The composition of the f-components is determined by the binary mediality property (cf. (27))

$$
\begin{aligned}
& \mathcal{M}_{\mathrm{Mor}}^{(2 \otimes)}\left[\mathrm{f}_{23}, \mathrm{~g}_{23}\right] \circ \mathcal{M}_{\mathrm{Mor}}^{(2 \otimes)}\left[\mathrm{f}_{12}, \mathrm{~g}_{12}\right]=\mathcal{M}_{\mathrm{Mor}}^{(2 \otimes)}\left[\mathrm{f}_{23} \circ \mathrm{f}_{12}, \mathrm{~g}_{23} \circ \mathrm{g}_{12}\right], \\
& \mathrm{f}_{i j}: X_{i} \rightarrow X_{j}, \mathrm{~g}_{i j}: Y_{i} \rightarrow Y_{j}, \mathrm{f}_{i j}, \mathrm{~g}_{i j} \in \operatorname{Mor} \mathscr{C}, \forall X_{i}, Y_{i} \in \mathrm{Ob} \mathscr{C}, i=1,2,3 .
\end{aligned}
$$

The identity of the tensor product satisfies

$$
\mathcal{M}_{\text {Mor }}^{(2 \otimes)}\left[\operatorname{id}_{X_{1}}, \mathrm{id}_{X_{2}}\right]=\mathrm{id}_{\mathcal{M}_{\mathrm{Ob}}^{(2 \otimes)}\left[X_{1}, X_{2}\right]} .
$$

We call a category $\mathscr{C}$ a strict (binary) semigroupal $[52,53]$ (or strictly associative semigroupal category [54], also, semi-monoidal [55]), if the bifunctor $\mathcal{M}^{(2 \otimes)}$ satisfies only (without unit objects and unitors) the binary associativity condition $\left(X_{1} \otimes X_{2}\right) \otimes X_{3}=X_{1} \otimes\left(X_{2} \otimes X_{3}\right)$ and $\left(f_{1} \otimes f_{2}\right) \otimes f_{3}=f_{1} \otimes\left(f_{2} \otimes f_{3}\right)$, where $X_{i} \in \operatorname{Ob} \mathscr{C}, f_{i} \in \operatorname{Mor} \mathscr{C}, i=1,2,3$ (also denoted by sSGCat). Strict associativity is the equivalence

$$
\begin{aligned}
\mathcal{M}_{\mathrm{Ob}}^{(2 \otimes)}\left[\mathcal{M}_{\mathrm{Ob}}^{(2 \otimes)}\left[X_{1}, X_{2}\right], X_{3}\right] & =\mathcal{M}_{\mathrm{Ob}}^{(2 \otimes)}\left[X_{1}, \mathcal{M}_{\mathrm{Ob}}^{(2 \otimes)}\left[X_{2}, X_{3}\right]\right], \\
\mathcal{M}_{\mathrm{Mor}}^{(2 \otimes)}\left[\mathcal{M}_{\mathrm{Mor}}^{(2 \otimes)}\left[\mathrm{f}_{1}, \mathrm{f}_{2}\right], \mathrm{f}_{3}\right] & =\mathcal{M}_{\mathrm{Mor}}^{(2 \otimes)}\left[\mathrm{f}_{1}, \mathcal{M}_{\mathrm{Mor}}^{(2 \otimes)}\left[\mathrm{f}_{2}, \mathrm{f}_{3}\right]\right] .
\end{aligned}
$$


Remark 3. Usually, only the first equation for the X-components is presented in the definition of associativity (and other properties), while the equation for the f-components is assumed to be satisfied "automatically" having the same form [13,56]. In some cases, the diagrams for $\mathcal{N}_{\mathrm{Ob}}^{(2 \otimes)}$ and $\mathcal{M}_{\mathrm{Mor}}^{(2 \otimes)}$ can fail to coincide and have different shapes, for instance, in the case of the dagger categories dealing with the "reverse" morphisms [57].

The associativity relations guarantee that in any product of objects or morphisms different ways of inserting parentheses lead to equivalent results (as for semigroups).

In the case of a non-strict semigroupal category SGCat (with no unit objects and unitors) $[52,54]$ (see, also, $[53,58,59]$ ) a collection of mappings can be introduced which are just the isomorphisms (associators) $A^{(3 \otimes)}=\left\{A_{O b}^{(3 \otimes)}, A_{\text {Mor }}^{(3 \otimes)}\right\}$ from the left side functor to the right side functor of (104) and (105) as

$$
\mathrm{A}_{\mathrm{Ob}}^{(3 \otimes)}\left(X_{1}, X_{2}, X_{3}\right): \mathcal{M}_{\mathrm{Ob}}^{(2 \otimes)}\left[\mathcal{M}_{\mathrm{Ob}}^{(2 \otimes)}\left[X_{1}, X_{2}\right], X_{3}\right] \stackrel{\simeq}{\rightarrow} \mathcal{M}_{\mathrm{Ob}}^{(2 \otimes)}\left[X_{1}, \mathcal{M}_{\mathrm{Ob}}^{(2 \otimes)}\left[X_{2}, X_{3}\right]\right],
$$

where $A_{\text {Mor }}^{(3 \otimes)}$ may be interpreted similarly to the diagonal in (98), because the associators are natural transformations [13] or trifunctorial isomorphisms (in the terminology of [54]). Now different ways of inserting parentheses in a product of $N$ objects give different results in the absence of conditions on the associator $A^{(3 \otimes)}$. However, if the associator $A^{(3 \otimes)}$ satisfies some consistency relations, they can give isomorphic results, such that the corresponding diagrams commute, which is the statement of the coherence theorem $[42,60]$. This can also be applied to SGCat, because it can be proved independently of existence of units [52-54]. It was shown [42] that it is sufficient to consider one commutative diagram using the associator (the associativity constraint) for two different rearrangements of parentheses for three tensor multiplications of four objects, giving the following isomorphism

$$
\mathcal{M}_{\mathrm{Ob}}^{(2 \otimes)}\left[\mathcal{M}_{\mathrm{Ob}}^{(2 \otimes)}\left[\mathcal{M}_{\mathrm{Ob}}^{(2 \otimes)}\left[X_{1}, X_{2}\right], X_{3}\right], X_{4}\right] \stackrel{\simeq}{\rightarrow} \mathcal{M}_{\mathrm{Ob}}^{(2 \otimes)}\left[X_{1}, \mathcal{M}_{\mathrm{Ob}}^{(2 \otimes)}\left[X_{2}, \mathcal{M}_{\mathrm{Ob}}^{(2 \otimes)}\left[X_{3}, X_{4}\right]\right]\right] .
$$

The constraint of associativity is called a pentagon axiom [13], such that the diagram

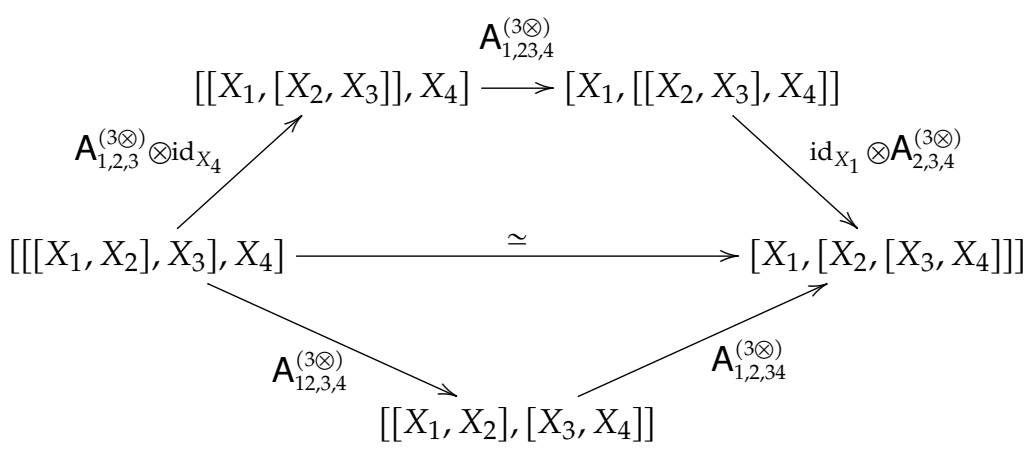

commutes. We omit $\mathcal{M}_{\mathrm{Ob}}^{(2 \otimes)}$ in diagrams by leaving the square brackets only and using the obvious subscripts in $A^{(3 \otimes)}$.

A similar condition for morphisms, but in another context (for $\mathrm{H}$-spaces), was presented in $[56,61]$. Note that there exists a different (but not alternative) approach to natural associativity without the use of the pentagon axiom [62].

The transition from the semigroupal non-strict category SGCat to the monoidal nonstrict category MonCat can be done in a way similar to passing from a semigroup to a monoid: by adding the unit object $E \in \mathrm{Ob} \mathscr{C}$ and the (right and left) unitors $\mathrm{U}_{(1)}^{(2 \otimes)}=$ $\left\{\mathrm{U}_{(1) \mathrm{Ob}^{\prime}}^{(2 \otimes)}, \mathrm{U}_{(1) \mathrm{Mor}}^{(2 \otimes)}\right\}$ and $\mathrm{U}_{(2)}^{(2 \otimes)}=\left\{\mathrm{U}_{(2) \mathrm{Ob}}^{(2 \otimes)}, \mathrm{U}_{(2) \mathrm{Mor}}^{(2 \otimes)}\right\}$ ("unit morphisms" which are functorial isomorphisms, natural transformations) [13] 


$$
\begin{aligned}
& \mathrm{U}_{(1) \mathrm{Ob}}^{(2 \otimes)}: \mathcal{M}_{\mathrm{Ob}}^{(2 \otimes)}[X, E] \stackrel{\simeq}{\rightrightarrows} X, \\
& \mathrm{U}_{(2) \mathrm{Ob}}^{(2 \otimes)}: \mathcal{M}_{\mathrm{Ob}}^{(2 \otimes)}[E, X] \stackrel{\simeq}{\rightrightarrows} X, \quad \forall X \in \mathrm{Ob} \mathscr{C},
\end{aligned}
$$

and $\mathrm{U}_{(1,2)}^{(2 \otimes)}$ Mor can be viewed as the diagonal in the diagram of naturality similar to (98). The unitors are connected with the associator $\mathrm{A}^{(3 \otimes)}$, such that the diagram (triangle axiom)

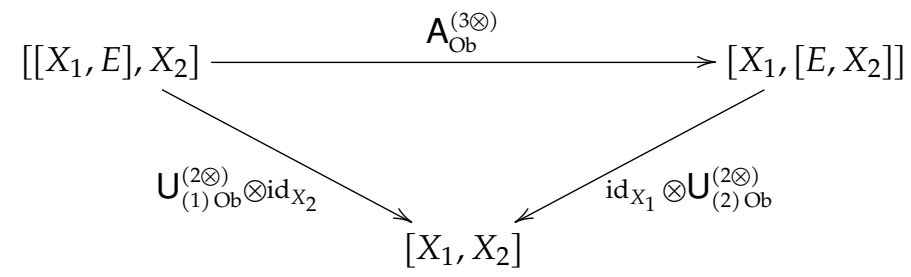

commutes.

Using the above, the definition of a binary non-strict monoidal category MonCat can be given as the 6-tuple $\left(\mathscr{C}, \mathcal{M} \mathcal{M}^{(2 \otimes)}, \mathrm{A}^{(3 \otimes)}, E, \mathrm{U}^{(2 \otimes)}\right)$ such that the pentagon axiom (108) and the triangle axiom (111) are satisfied [13,42] (see, also, [60,63]).

The following "normalizing" relations for the unitors of a monoidal non-strict category

$$
\mathrm{U}_{(1) \mathrm{Ob}}^{(2 \otimes)}(E)=\mathrm{U}_{(2) \mathrm{Ob}}^{(2 \otimes)}(E),
$$

can be proven [17], as well as that the diagrams
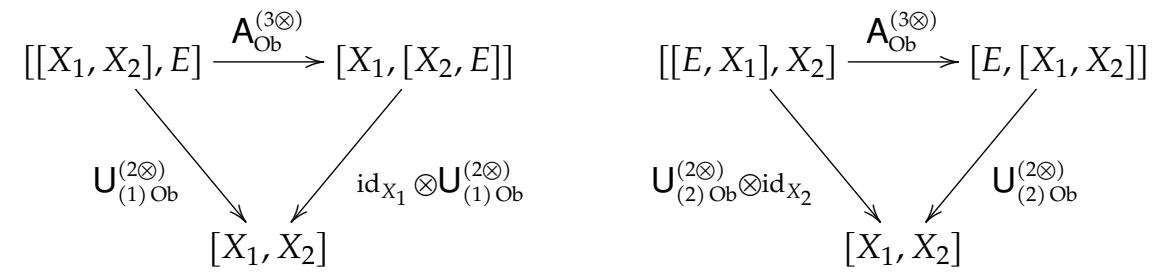

commute.

The coherence theorem $[41,42]$ proves that any diagram in a non-strict monoidal category, which can be built from an associator satisfying the pentagon axiom (108) and unitors satisfying the triangle axiom (111), commutes. Another formulation [13] states that every monoidal non-strict category is (monoidally) equivalent to a monoidal strict one (see also [64]).

Thus, it is important to prove analogs of the coherence theorem for various existing generalizations of categories (having weak modification of units $[55,65,66]$ and from the "periodic table" of higher categories [67]) as well as for further generalizations (e.g., $n$-ary ones below).

\section{Polyadic Tensor Categories}

The arity of the additional multiplication in a category (the tensor product) was previously taken to be binary. Here we introduce categories with tensor multiplication which "remind" $n$-ary semigroups, $n$-ary monoids and $n$-ary groups [15,16] (see, also [68]), i.e., we provide the categorification $[50,69]$ of "higher-arity" structures according to the Baez-Dolan microcosm principle [47]. In our considerations we use the term "tensor category" in a wider context, because it can include not only binary monoid-like structures and their combinations but also $n$-ary-like algebraic structures. It is important to note that our construction is different from other higher generalizations of categories, such as 2-categories [70] and bicategories [71], $n$-categories [72,73] and $n$-categories of $n$-groups [74], multicategories [75-77], $n$-tuple categories and multiple categories [78], iterated ( $n$-fold) monoidal categories [79], iterated icons [80], and obstructed categories [81,82]. 
We introduce the categorification of "higher-arity" structures like [20] and consider their properties, some of them are different from the binary case (as in $n$-ary (semi)groups and $n$-ary monoids). Also, the terms " $k$-ary algebraic category" and " $k$-ary category" appeared in $[83,84]$, respectively, but they describe different constructions.

Let $\mathscr{C}$ be a category [13], we introduce an additional multiplication as an $n$-ary tensor product similar to $[20,38]$.

Definition 28. An n-ary tensor product in a category $\mathscr{C}$ is an n-ary functor

$$
\mathcal{M}^{(n \otimes)}: \overbrace{\mathscr{C} \times \ldots \times \mathscr{C}}^{n} \rightarrow \mathscr{C}
$$

having the component form $\mathcal{M}^{(n \otimes)}=\left\{\mathcal{M}_{\mathrm{Ob}}^{(n \otimes)}, \mathcal{M}_{\mathrm{Mor}}^{(n \otimes)}\right\}$ where the f-component $\mathcal{M}_{\mathrm{Mor}}^{(n \otimes)}$ is

$$
\begin{aligned}
& \mathcal{M}_{\mathrm{Mor}}^{(n \otimes)}\left[\mathrm{f}_{11^{\prime}}, \mathrm{f}_{22^{\prime}}, \ldots \mathrm{f}_{n n^{\prime}}\right]=\mathcal{M}_{\mathrm{Ob}}^{(n \otimes)}\left[X_{1}, X_{2}, \ldots X_{n}\right] \rightarrow \mathcal{M}_{\mathrm{Ob}}^{(n \otimes)}\left[X_{1}^{\prime}, X_{2}^{\prime}, \ldots X_{n}^{\prime}\right] \\
& \mathrm{f}_{i i^{\prime}}: X_{i} \rightarrow X_{i}^{\prime}, \mathrm{f}_{i i^{\prime}} \in \operatorname{Mor} \mathscr{C}, \forall X_{i}, X_{i}^{\prime} \in \mathrm{Ob} \mathscr{C}, i=1, \ldots, n .
\end{aligned}
$$

The $n$-ary composition of the $\mathrm{f}$-components (morphism products of length $n$ ) is determined by the $n$-ary mediality property (cf. (57))

$$
\begin{aligned}
& \mathcal{M}_{\text {Mor }}^{(n \otimes)}\left[\mathrm{f}^{(1,1)}, \mathrm{f}^{(1,2)}, \ldots, \mathrm{f}^{(1, n)}\right] \circ \ldots \circ \mathcal{M}{ }_{\text {Mor }}^{(n \otimes)}\left[\mathrm{f}^{(n, 1)}, \mathrm{f}^{(n, 2)}, \ldots, \mathrm{f}^{(n, n)}\right], \\
& =\mathcal{M}_{M}^{(n \otimes)}\left[\mathrm{f}^{(1,1)} \circ \mathrm{f}^{(2,1)} \circ \ldots \circ \mathrm{f}^{(n, 1)}, \ldots, \mathrm{f}^{(1, n)} \circ \ldots \circ \mathrm{f}^{(n, n)}\right], \\
& \mathrm{f}^{(i, j)} \in \operatorname{Mor} \mathscr{C}, \quad i, j=1,2 \ldots, n .
\end{aligned}
$$

The identity morphism of the $n$-ary tensor product satisfies

$$
\mathcal{M}_{\text {Mor }}^{(n \otimes)}\left[\operatorname{id}_{X_{1}}, \operatorname{id}_{X_{2}}, \ldots, \operatorname{id}_{X_{n}}\right]=\operatorname{id}_{\mathcal{M}_{\mathrm{Ob}}^{(n \otimes)}\left[X_{1}, X_{2} \ldots, X_{n}\right]} .
$$

Definition 29. An n-ary tensor product $\mathcal{M}^{(n \otimes)}$ which can be constructed from a binary tensor product $\mathcal{M}^{\prime(2 \otimes)}$ by successive (iterative) repetitions is called an arity-reduced tensor product, by analogy with the "derived n-ary group" [15,16], and otherwise it is called an arity-nonreduced tensor product.

Categories containing iterations of the binary tensor product were considered in $[79,80]$. We will mostly be interested in the arity-nonreducible tensor products and their corresponding categories.

Definition 30. A polyadic (n-ary) "magmatic" tensor category is $\left(\mathscr{C}, \mathcal{M}^{(n \otimes)}\right)$, where $\mathcal{M}^{(n \otimes)}$ is an n-ary tensor product (functor (114)), and it is called an arity-reduced category or aritynonreduced category depending on its tensor product.

\subsection{Polyadic Semigroupal Categories}

We call sequences of objects and morphisms X-polyads and f-polyads [16] and denote them $\mathbf{X}$ and $\mathbf{f}$, respectively (as in (53)).

Definition 31. The n-ary functor $\mathcal{M}^{(n \otimes)}$ is totally (n-ary) associative, if it satisfies the following $(n-1)$ pairs of $X$ equivalences

$$
\mathcal{M}_{\mathrm{Ob}}^{(n \otimes)}\left[\mathbf{X}, \mathcal{M}_{\mathrm{Ob}}^{(n \otimes)}[\mathbf{Y}], \mathbf{Z}\right]=\text { equivalent }
$$

where $\mathbf{X}, \mathbf{Y}, \mathbf{Z}$ are $\mathbf{X}$-polyads of the necessary length, and the total length of each $(\mathbf{X}, \mathbf{Y}, \mathbf{Z})$-polyad is $2 n-1$, while the internal tensor products in (118) can be on any of the $n$ places. 
Example 4. In the ternary case $(n=3)$ the total associativity for the X-polyads of the length $5=2 \cdot 3-1$ gives $2=3-1$ pairs of equivalences

$$
\begin{aligned}
\mathcal{M}_{\mathrm{Ob}}^{(3 \otimes)}\left[\mathcal{M}_{\mathrm{Ob}}^{(3 \otimes)}\left[X_{1}, X_{2}, X_{3}\right], X_{4}, X_{5}\right] & =\mathcal{M}_{\mathrm{Ob}}^{(3 \otimes)}\left[X_{1}, \mathcal{M}_{\mathrm{Ob}}^{(3 \otimes)}\left[X_{2}, X_{3}, X_{4}\right], X_{5}\right] \\
& =\mathcal{M}_{\mathrm{Ob}}^{(3 \otimes)}\left[X_{1}, X_{2}, \mathcal{\mathcal { M } _ { \mathrm { Ob } } ^ { ( 3 \otimes ) }}\left[X_{3}, X_{4}, X_{5}\right]\right], \\
\forall X_{i} & \in \mathrm{Ob} \mathscr{C}, \quad \forall f_{i} \in \operatorname{Mor} \mathscr{C}, \quad i=1, \ldots, 5 .
\end{aligned}
$$

Definition 32. A category $\left(\mathscr{C}, \mathcal{M}^{(n \otimes)}\right)$ is called a polyadic (n-ary) strict semigroupal category $\mathrm{sSGCat}_{n}$, if the bifunctor $\mathcal{M}^{(n \otimes)}$ satisfies objects and unitors) the n-ary associativity condition (118).

Thus, in a polyadic strict semigroupal category for any (allowed, i.e., having the size $k(n-1)+1, \forall k \in \mathbb{N}$, where $k$ is the number of $n$-ary tensor multiplications) product of objects (or morphisms), all different ways of inserting parentheses give equivalent results (as for $n$-ary semigroups).

\section{2. n-Ary Coherence}

As in the binary case (106), the transition to non-strict categories results in the consideration of independent isomorphisms instead of the equivalence (118).

Definition 33. The $(n-1)$ pairs of $X$ and $f$ isomorphisms $A^{(2 n-1) \otimes}=\left\{A_{O b}^{(2 n-1) \otimes}, A_{M o r}^{(2 n-1) \otimes}\right\}$ such that

$$
\mathrm{A}_{i, \mathrm{Ob}}^{(2 n-1) \otimes}: \mathcal{M}_{\mathrm{Ob}}^{(n \otimes)}\left[\mathbf{X}, \mathcal{M}_{i, \mathrm{Ob}}^{(n \otimes)}[\mathbf{Y}], \mathbf{Z}\right] \stackrel{\simeq}{\rightarrow} \mathcal{M}_{\mathrm{Ob}}^{(n \otimes)}\left[\mathbf{X}, \mathcal{M}_{i+1, \mathrm{Ob}}^{(n \otimes)}[\mathbf{Y}], \mathbf{Z}\right]
$$

are called $n$-ary associators being $(2 n-1)$-place natural transformations, where $\mathrm{A}_{\mathrm{Mor}}^{(2 n-1) \otimes}$ may be viewed as corresponding diagonals as in (98). Here $i=1, \ldots, n-1$ is the place of the internal brackets.

In the ternary case $(n=3)$ we have $2=3-1$ pairs of the ternary associators

$$
\mathrm{A}_{1, \mathrm{Ob}}^{(5 \otimes)}: \mathcal{M}_{\mathrm{Ob}}^{(3 \otimes)}\left[\mathcal{M}_{\mathrm{Ob}}^{(3 \otimes)}\left[X_{1}, X_{2}, X_{3}\right], X_{4}, X_{5}\right] \stackrel{\sim}{\rightarrow} \mathcal{M}_{\mathrm{Ob}}^{(3 \otimes)}\left[X_{1}, \mathcal{M}_{\mathrm{Ob}}^{(3 \otimes)}\left[X_{2}, X_{3}, X_{4}\right], X_{5}\right],
$$

and

$$
\mathrm{A}_{2, \mathrm{Ob}}^{(5 \otimes)}: \mathcal{M}_{\mathrm{Ob}}^{(3 \otimes)}\left[X_{1}, \mathcal{M}_{\mathrm{Ob}}^{(3 \otimes)}\left[X_{2}, X_{3}, X_{4}\right], X_{5}\right] \stackrel{\cong}{\rightrightarrows} \mathcal{M}_{\mathrm{Ob}}^{(3 \otimes)}\left[X_{1}, X_{2}, \mathcal{M}_{\mathrm{Ob}}^{(3 \otimes)}\left[X_{3}, X_{4}, X_{5}\right]\right] .
$$

It is now definite that different ways of inserting parentheses in a product of $N$ objects will give different results (the same will be true for morphisms as well), if we do not impose constraints on the associators. We anticipate that we will need (as in the binary case (107)) only one more (i.e., three) tensor multiplication than appears in the associativity conditions (118) to make a commutative diagram for the following isomorphism of $3 \cdot(n-1)+1=3 n-2$ objects

$$
\begin{aligned}
& \mathcal{M}_{\mathrm{Ob}}^{(n \otimes)}\left[\mathcal{M}_{\mathrm{Ob}}^{(n \otimes)}\left[\mathcal{M}_{\mathrm{Ob}}^{(n \otimes)}\left[X_{1}, \ldots, X_{n}\right], X_{n+1}, \ldots, X_{2 n-1}\right], X_{2 n}, \ldots, X_{3 n-2}\right] \\
& \stackrel{\cong}{\rightarrow} \mathcal{M}_{\mathrm{Ob}}^{(n \otimes)}\left[X_{1}, \ldots, X_{n-1}, \mathcal{M}_{\mathrm{Ob}}^{(n \otimes)}\left[X_{n}, \ldots, X_{2 n-2}, \mathcal{M}_{\mathrm{Ob}}^{(n \otimes)}\left[X_{2 n-1}, \ldots, X_{3 n-2}\right]\right]\right] .
\end{aligned}
$$

Conjecture 1 ( $N$-ary coherence). If the $n$-ary associator $\mathrm{A}^{(2 n-1) \otimes}$ satisfies such $n$-ary coherence conditions that the isomorphism (123) takes place, then any diagram containing $A^{(2 n-1) \otimes}$ together with the identities (117) commutes. 
The $n$-ary coherence conditions are described by a " $\left(n^{2}+1\right)$-gon", which is the pentagon (108) for $n=2$ (for classification of " $N$-gons" see, e.g., [85]).

Definition 34. A category $\left(\mathscr{C}, \mathcal{M}^{(n \otimes)}\right)$ is called a polyadic (n-ary) non-strict semigroupal category $\mathrm{sSGCat}_{n}$, if the bifunctor $\mathcal{M}^{(n \otimes)}$ satisfies the n-ary coherence.

Example 5. In the ternary case $n=3$ we have 2 pairs of 5-place associators (121)-(122) $A_{1}^{(5 \otimes)}$ and $A_{2}^{(5 \otimes)}$ which act on $7=3 \cdot 3-2$ objects (123). We consider the diagram for objects only, then the associativity constraint for the associators $\mathrm{A}_{1, \mathrm{Ob}}^{(5 \otimes)}$ and $\mathrm{A}_{2, \mathrm{Ob}}^{(5 \otimes)}$ will be a decagon axiom requiring that the diagram

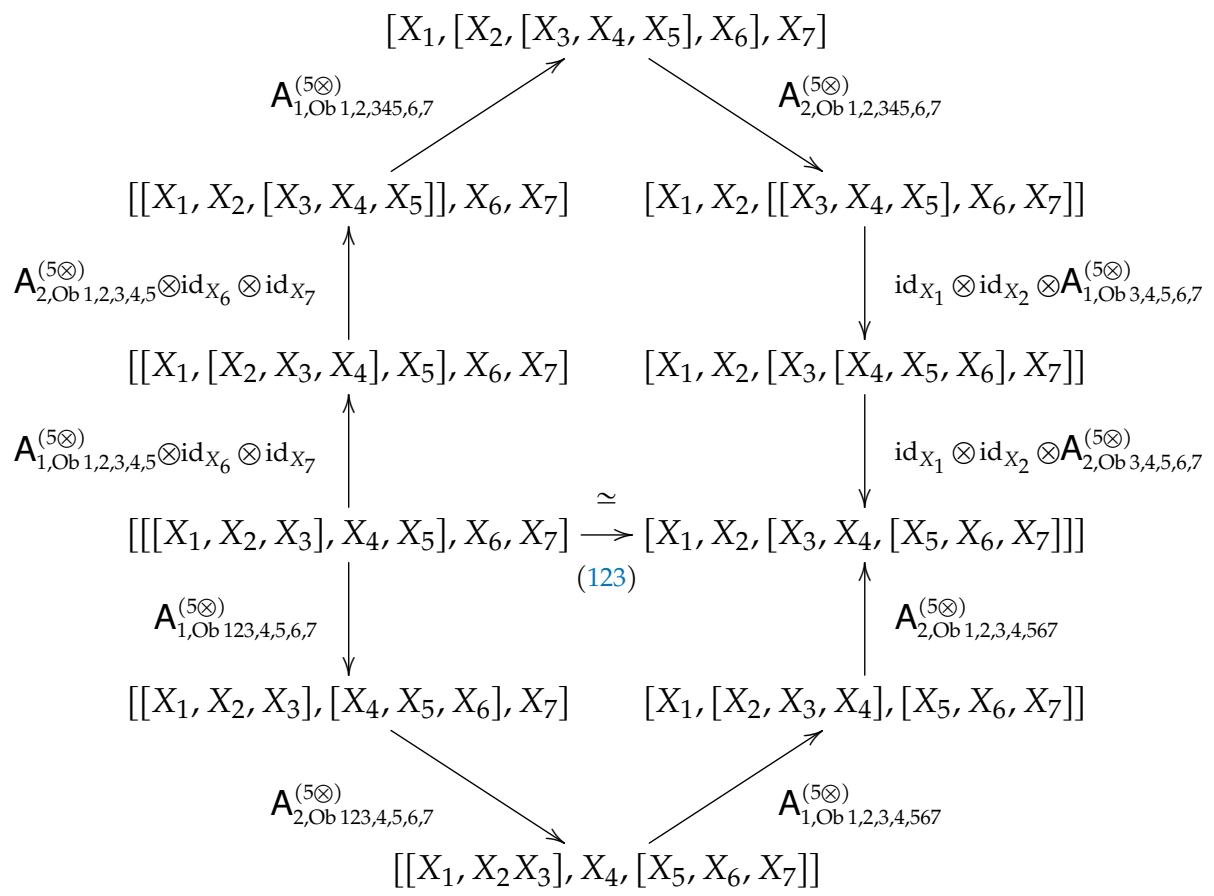

commutes (cf. (124) and the pentagon axiom (108) for binary non-strict tensor categories).

\section{9. $n$-Ary Units, Unitors and Quertors}

Introducing $n$-ary (polyadic) analogs of units and unitors is nontrivial, because in $n$-ary (polyadic) structures there are various possibilities: one unit, many units, all elements are units or there are no units at all (see, e.g., for $n$-ary groups $[15,16,68]$, and for $n$-ary monoids [86]). A similar situation is expected in the category theory after proper categorification $[46,50,69]$ of $n$-ary (polyadic) structures.

\subsection{Polyadic Monoidal Categories}

Let $\left(\mathscr{C}, \mathcal{M}^{(n \otimes)}, \mathrm{A}^{(2 n-1) \otimes}\right)$ be an $n$-ary non-strict semigroupal category $\mathrm{SGCat}_{n}$ (see Definition 32) with $n$-ary tensor product $\mathcal{M}^{(n \otimes)}$ and the associator $\mathrm{A}^{(2 n-1) \otimes}$ satisfying $n$-ary coherence. If a category has a unit neutral sequence of objects $\mathbf{E}_{(n-1)}=\left(E_{1}, \ldots, E_{i}\right)$, $E_{i} \in \mathrm{Ob} \mathscr{C}, i=1, \ldots, n-1$, we call it a unital category. Note that the unit neutral sequence may not be unique. If all $E_{i}$ coincide $E_{i}=E \in \mathrm{Ob} \mathscr{C}$, then $E$ is called a unit object of $\mathscr{C}$. The $n$-ary unitors $\mathrm{U}_{(i)}^{(n \otimes)}, i=1, \ldots, n$ (n-ary "unit morphisms" being natural transformations) are defined by

$$
\mathrm{U}_{(i) \mathrm{Ob}}^{(n \otimes)}: \mathcal{M}_{\mathrm{Ob}}^{(n \otimes)}\left[E_{1}, \ldots E_{i-1}, X, E_{i+1}, \ldots E_{n}\right] \stackrel{\sim}{\rightrightarrows} X, \quad \forall X, E_{i} \in \mathrm{Ob} \mathscr{C}, i=1, \ldots, n-1 .
$$


The $n$-ary unitors $\mathrm{U}_{(i)}^{(n \otimes)}$ are compatible with the $n$-ary associators $\mathrm{A}^{(2 n-1) \otimes}$ by the analog of the triangle axiom (111). In the binary case (109)-(110), we have $U_{(1)}^{(2 \otimes)}=R^{(2 \otimes)}$, $\mathrm{U}_{(2)}^{(2 \otimes)}=\mathrm{L}^{(2 \otimes)}$.

Definition 35. A polyadic (n-ary) non-strict monoidal category MonCat $_{n}$ is a polyadic (n-ary) non-strict semigroupal category SGCat ${ }_{n}$ endowed with a unit neutral sequence $\mathbf{E}_{(n-1)}$ and $n$ unitors $\mathrm{U}_{(i)}^{(n \otimes)}, i=1, \ldots, n$, that is a 5-tuple $\left(\mathscr{C}, \mathcal{M}^{(n \otimes)}, \mathbf{A}^{(n \otimes)}, \mathbf{E}_{(n-1)}, \mathrm{U}^{(n \otimes)}\right)$ satisfying the " $\left(n^{2}+1\right)$ gon" axiom for the $(n-1)$ associators $\mathrm{A}_{(i)}^{(2 n-1) \otimes}$ and the triangle axiom (the analog of (111)) for the unitors and associators compatibility condition.

Example 6. If we consider the ternary non-strict monoidal category MonCat 3 with one unit object $E \in \mathrm{Ob} \mathscr{C}$, then we have 2 associators $\mathrm{A}_{1}^{(5 \otimes)}$ and $\mathrm{A}_{2}^{(5 \otimes)}$ satisfying the decagon axiom (124) and 3 unitors

$$
\begin{aligned}
& \mathrm{U}_{(1) \mathrm{Ob}}^{(3 \otimes)}: \mathcal{M}_{\mathrm{Ob}}^{(3 \otimes)}[X, E, E] \stackrel{\simeq}{\rightarrow} X, \\
& \mathrm{U}_{(2) \mathrm{Ob}}^{(3 \otimes)}: \mathcal{M}_{\mathrm{Ob}}^{(3 \otimes)}[E, X, E] \stackrel{\simeq}{\rightarrow} X, \\
& \mathrm{U}_{(3) \mathrm{Ob}}^{(3 \otimes)}: \mathcal{M}_{\mathrm{Ob}}^{(3 \otimes)}[E, E, X] \stackrel{\simeq}{\rightarrow} X, \quad \forall X \in \mathrm{Ob} \mathscr{C},
\end{aligned}
$$

which satisfy the "normalizing" conditions $\mathrm{U}_{(i)}^{(3 \otimes)} \mathrm{Ob}(E)=E, i=1,2,3$ and the ternary analog of the triangle axiom (111), such that the diagram

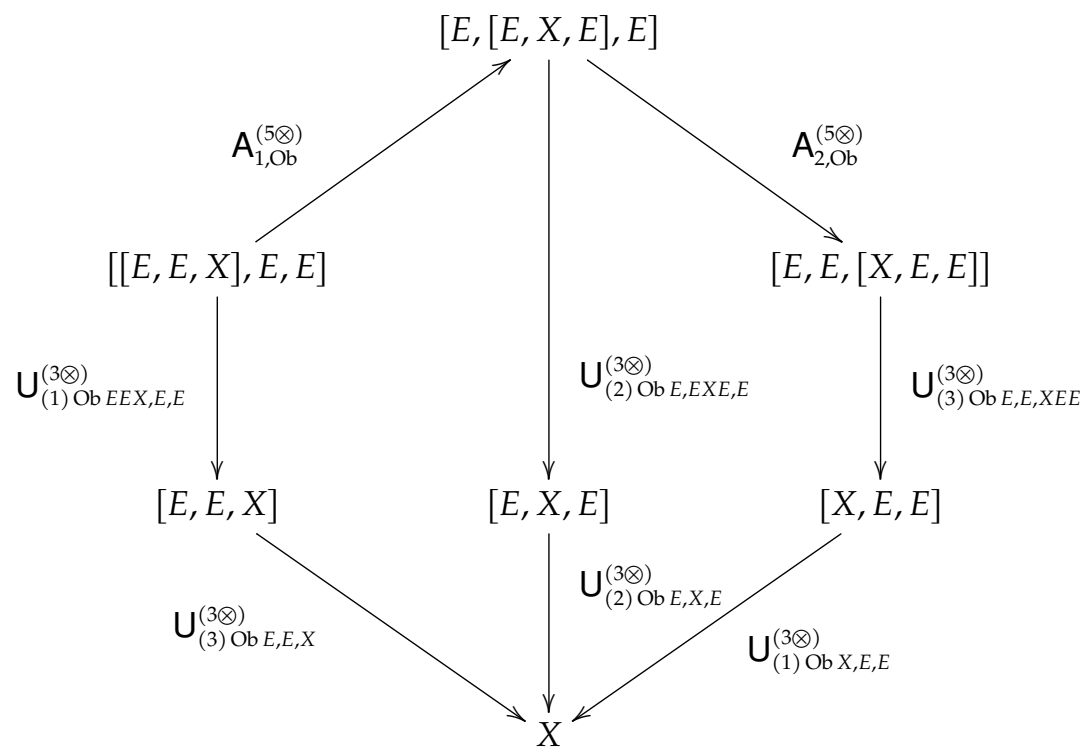

commutes.

\subsection{Polyadic Nonunital Groupal Categories}

The main result of $n$-ary group theory $[15,16]$ is connected with units and neutral polyads: if they exist, then such $n$-ary group is reducible to a binary group. A similar statement can be true in some sense for categories.

Conjecture 2. If a polyadic (n-ary) tensor category has unit object and unitors, it can be arityreducible to a binary category, such that the n-ary product can be obtained by iterations of the binary tensor product. 
Therefore, it would be worthwhile to introduce and study non-reducible polyadic tensor categories which do not possess unit objects and unitors at all. This can be done by "categorification" of the querelement concept [15]. Recall that, for instance, in a ternary group $\left\langle G \mid \mu_{3}\right\rangle$ for an element $g \in G$ a querelement $\bar{g}$ is uniquely defined by $\mu_{3}[g, g, \bar{g}]=g$, which can be treated as a generalization of the inverse element concept to the $n$-ary case. The mapping $g \rightarrow \bar{g}$ can be considered as an additional unary operation (queroperation) in the ternary (and $n$-ary) group, while viewing it as an abstract algebra [87] such that the notion of the identity is not used. The (binary) category of $n$-ary groups and corresponding functors were considered in [88-90].

Let $\left(\mathscr{C}, \mathcal{M}^{(n \otimes)}, \mathrm{A}^{(2 n-1) \otimes}\right)$ be a polyadic (n-ary) non-strict semigroupal category, where $\mathcal{M}^{(n \otimes)}$ is the $n$-ary tensor product, and $A^{(2 n-1) \otimes}$ is the associator making the " $\left(n^{2}+1\right)$-gon" diagram of $n$-ary coherence commutative. We propose a "categorification" analog of the queroperation to be a covariant endofunctor of $\mathscr{C}$.

Definition 36. A querfunctor $Q: \mathscr{C} \rightarrow \mathscr{C}$ is an endofunctor of $\mathscr{C}$ sending $Q_{\mathrm{Ob}}(X)=\bar{X}$ and $Q_{\text {Mor }}(\mathrm{f})=\overline{\mathrm{f}}$, where $\bar{X}$ and $\overline{\mathrm{f}}$ are the querobject and the quermorphism of $\mathrm{X}$ and $\mathrm{f}$, respectively, such that the $i$ diagrams $(i=1, \ldots, n)$

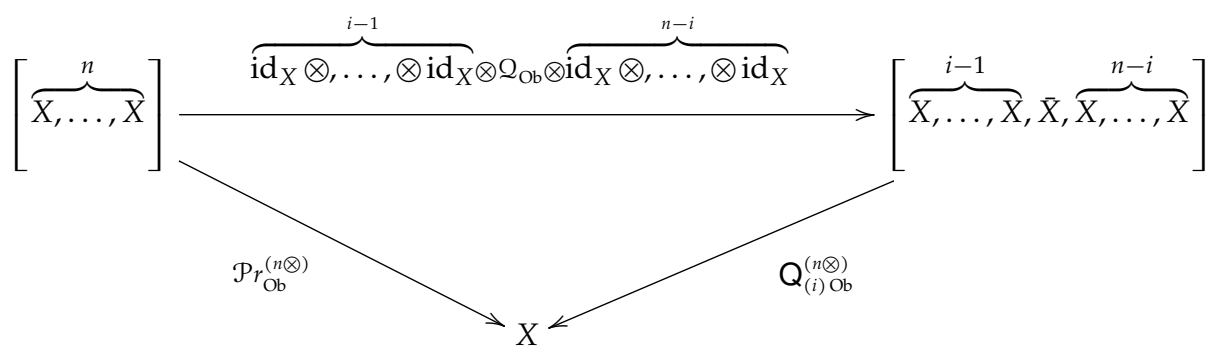

commute (and analogously for morphisms), where $\mathrm{Q}_{(i)}^{(n \otimes)}$ are quertors

$$
\mathrm{Q}_{(i) \mathrm{Ob}}^{(n \otimes)}: \mathcal{M}_{\mathrm{Ob}}^{(n \otimes)}[\overbrace{X, \ldots, X}^{i-1}, \bar{X}, \overbrace{X, \ldots, X}^{n-i}] \stackrel{\simeq}{\rightrightarrows} X, \quad \forall X \in \mathrm{Ob} \mathscr{C}, i=1, \ldots, n,
$$

and $\mathcal{P}_{r}^{(n \otimes)}: \mathscr{C}^{n \otimes} \rightarrow \mathscr{C}$ is the projection. The action on morphisms $\mathrm{Q}_{(i) \text { Mor }}^{(n \otimes)}$ can be found using the diagonal arrow in the corresponding natural transformation, as in (98).

Example 7. In the ternary case we have (for objects) the querfunctor $Q_{\mathrm{Ob}}(X)=\bar{X}$ and three quertor isomorphisms

$$
\begin{aligned}
& \mathrm{Q}_{(1) \mathrm{Ob}}^{(3 \otimes)}: \mathcal{M}_{\mathrm{Ob}}^{(3 \otimes)}[\bar{X}, X, X] \stackrel{\simeq}{\rightarrow} X, \\
& \mathrm{Q}_{(2) \mathrm{Ob}}^{(3 \otimes)}: \mathcal{M}_{\mathrm{Ob}}^{(3 \otimes)}[X, \bar{X}, X] \stackrel{\simeq}{\rightarrow} X, \\
& \mathrm{Q}_{(3) \mathrm{Ob}}^{(3 \otimes)}: \mathcal{M}_{\mathrm{Ob}}^{(3 \otimes)}[X, X, \bar{X}] \stackrel{\simeq}{\rightarrow} X, \quad \forall X \in \mathrm{Ob} \mathscr{C} .
\end{aligned}
$$

The three quertors $\mathrm{Q}_{(i) \mathrm{Ob}}^{(3 \otimes)}$ and the querfunctor $\mathrm{Q}$ are connected with two ternary associators $\mathrm{A}_{1, \mathrm{Ob}}^{(5 \otimes)}, \mathrm{A}_{2, \mathrm{Ob}}^{(5 \otimes)}$ (121) and (122) such that the following diagram 


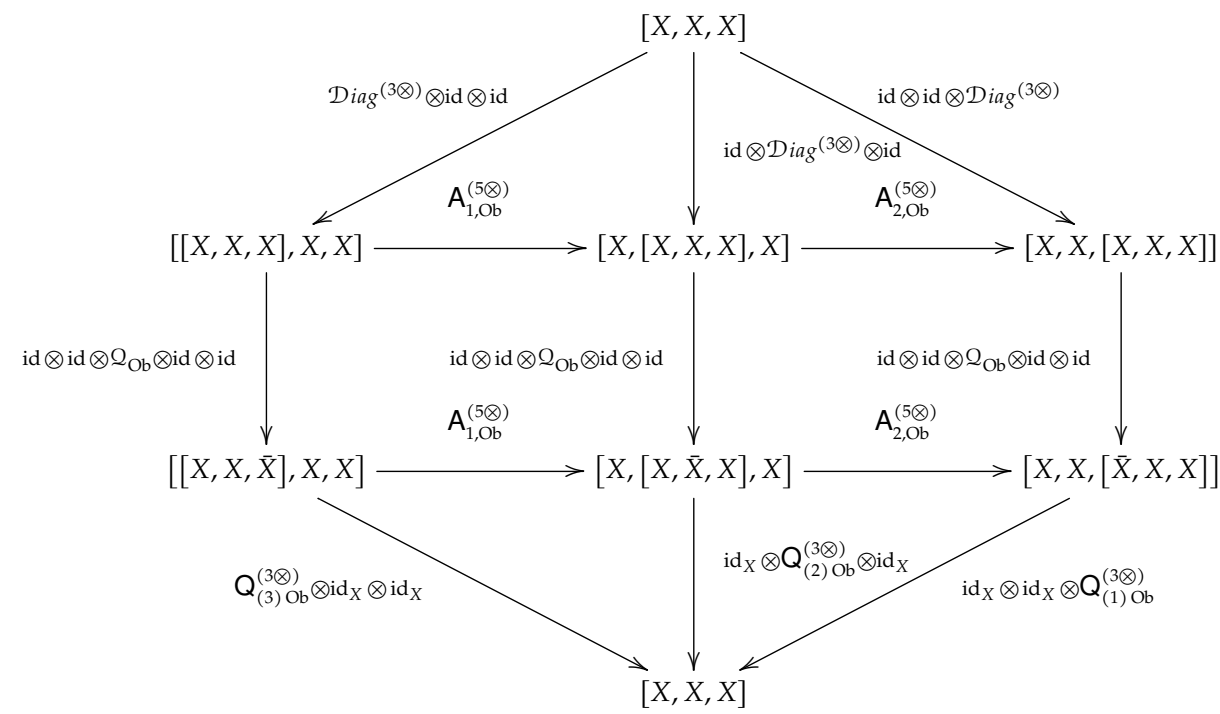

commutes, where $\operatorname{Diag}^{(n \otimes)}: \mathscr{C} \rightarrow \mathscr{C}^{n \otimes}$ is the diagonal.

Definition 37. A nonunital non-strict groupal category is

$$
\operatorname{GCat}_{n}=\left(\mathscr{C}, \mathcal{M}^{(n \otimes)}, \mathrm{A}^{(2 n-1) \otimes}, \mathcal{Q}, \mathrm{Q}^{(n \otimes)}\right),
$$

i.e., a polyadic non-strict semigroupal category $\mathrm{SGCat}_{n}$ equipped with the querfunctor $\mathrm{Q}$ and the quertors $\mathrm{Q}^{(n \otimes)}$ satisfying (130).

Conjecture 3. There exist polyadic nonunital non-strict groupal categories which are arity-nonreducible (see Definition 30), and so their n-ary tensor product cannot be presented in the form of binary tensor product iterations.

\section{Braided Tensor Categories}

The next step in the investigation of binary tensor categories is consideration of the tensor product "commutativity" property. The tensor product can be "commutative" such that for a tensor category $\mathscr{C}$ there exists the equivalence $X \otimes Y=Y \otimes X, \forall X, Y, \in \mathrm{Ob} \mathscr{C}$, and such tensor categories are called symmetric [13]. By analogy with associativity, one can introduce non-strict "commutativity", which leads to the notion of a braided (binary) tensor category and the corresponding coherence theorems [17]. Various generalizations of braiding were considered in [81,91,92], and their higher versions are found, e.g., in [93-95].

\subsection{Braided Binary Tensor Categories}

Let $\left(\mathscr{C}, \mathcal{M}^{(2 \otimes)}, A^{(3 \otimes)}\right)$ be a non-strict semigroupal category with the bifunctor $\mathcal{M}^{(2 \otimes)}$ and the associator $A^{(3 \otimes)}(106)$ satisfying the pentagon axiom (108) [52,54].

Definition 38. $A$ (binary) braiding $\mathrm{B}^{(2 \otimes)}=\left\{\mathrm{B}_{\mathrm{Ob}}^{(2 \otimes)}, \mathrm{B}_{\text {Mor }}^{(2 \otimes)}\right\}$ of a semigroupal category SGCat $_{2}$ is a natural transformation of the bifunctor $\mathcal{M}^{(2 \otimes)}$ (bifunctorial isomorphism) such that

$$
\mathrm{B}_{\mathrm{Ob}}^{(2 \otimes)}: \mathcal{M}_{\mathrm{Ob}}^{(2 \otimes)}\left[X_{1}, X_{2}\right] \stackrel{\simeq}{\rightarrow} \mathcal{M}_{\mathrm{Ob}}^{(2 \otimes)}\left[X_{2}, X_{1}\right], \quad \forall X_{i} \in \mathrm{Ob} \mathscr{C}, i=1,2,
$$

and the action on morphisms $\mathrm{B}_{\mathrm{Mor}}^{(2 \otimes)}$ may be interpreted as a diagonal, similarly to (98).

Definition 39. A non-strict semigroupal category endowed with a binary braiding is called a (binary) braided semigroupal category bSGCat $_{2}\left(\mathscr{C}, \mathcal{M}^{(2 \otimes)}, \mathrm{A}^{(3 \otimes)}, \mathrm{B}^{(2 \otimes)}\right)$. 
The braiding $\mathrm{B}^{(2 \otimes)}$ is connected with the associator $\mathrm{A}^{(3 \otimes)}$ by the hexagon identity

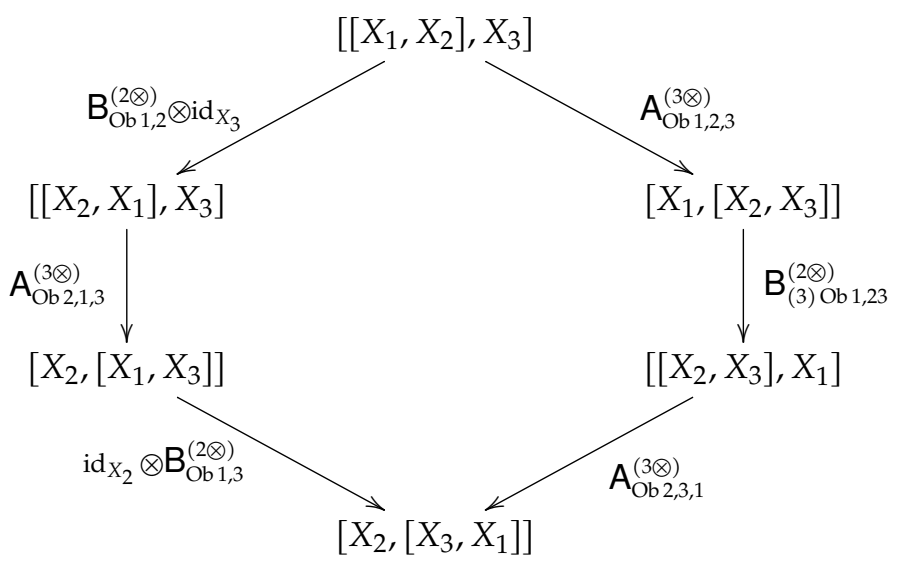

for objects, and similarly for the inverse associator.

Definition 40. A symmetric braided semigroupal category sbSGCat 2 has the "invertible" braiding

$$
\begin{aligned}
\mathrm{B}_{\mathrm{Ob} X_{1}, X_{2}}^{(2 \otimes)} \circ \mathrm{B}_{\mathrm{Ob} X_{2}, X_{1}}^{(2 \otimes)} & =\mathrm{id}_{X_{1} \otimes X_{2}} \quad \text { or } \\
\mathrm{B}_{\mathrm{Ob} X_{2}, X_{1}}^{(2 \otimes)} & =\mathrm{B}_{\mathrm{Ob} X_{1}, X_{2}}^{(2 \otimes),-1} \quad \forall X_{i} \in \mathrm{Ob} \mathscr{C}
\end{aligned}
$$

A von Neumann regular generalization [96] (weakening) of (138) leads to

Definition 41. A (von Neumann) regular braided semigroupal category is defined by a braiding which satisfies $[92,97]$

$$
\mathrm{B}_{\mathrm{Ob} X_{1}, X_{2}}^{(2 \otimes)} \circ \mathrm{B}_{\mathrm{Ob} X_{1}, X_{2}}^{*(2 \otimes)} \circ \mathrm{B}_{\mathrm{Ob} X_{1}, X_{2}}^{(2 \otimes)}=\mathrm{B}_{\mathrm{Ob} X_{1}, X_{2}}^{(2 \otimes)}
$$

where $\mathrm{B}_{\mathrm{Ob} X_{1}, X_{2}}^{*(2 \otimes)}$ is a generalized inverse $[98,99]$ of $\mathrm{B}_{\mathrm{Ob} X_{1}, X_{2}}^{(2 \otimes)}$, and such that $\mathrm{B}_{\mathrm{Ob} X_{1}, X_{2}}^{*(2 \otimes)} \neq \mathrm{B}_{\mathrm{Ob} X_{1}, X_{2}}^{(2 \otimes),-1}$ (cf. (139)).

Proposition 14. If the (binary) braided semigroupal category is strict (the associator becomes the equivalence (104) and (105), and we can omit internal brackets), then the diagram

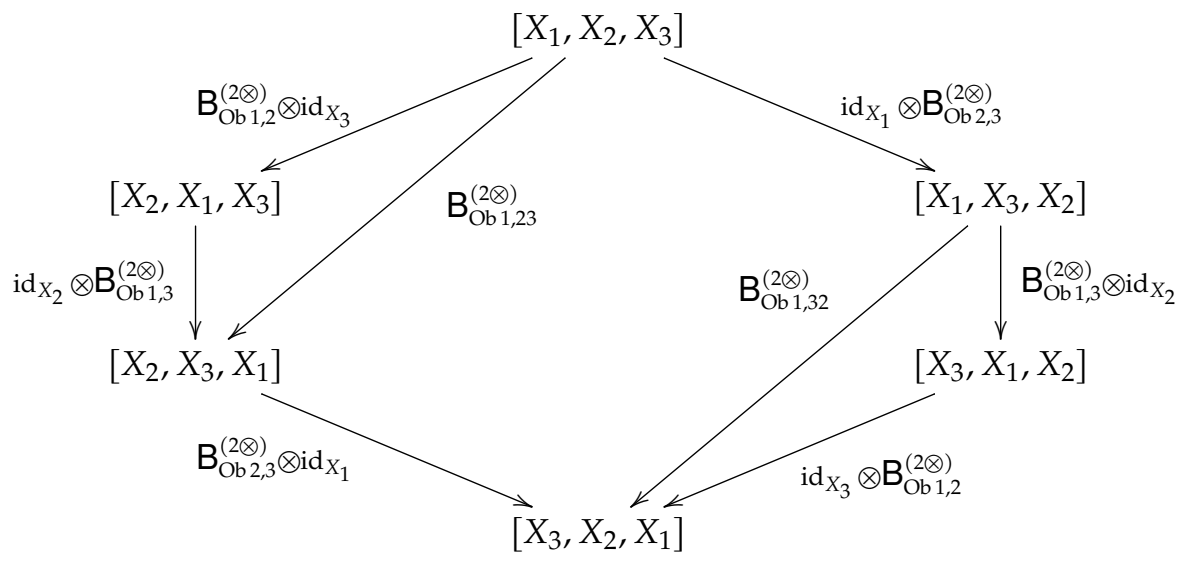

commutes [56,61]. 
Proof. The triangles commute due to the hexagon identity (137) and the internal rectangle commutes, because the binary braiding $\mathrm{B}^{(2 \otimes)}$ is a natural transformation (bifunctorial isomorphism).

Omitting indices (141) becomes the Yang-Baxter equation in terms of tensor products [100] (or the binary braid group relation —-for their difference, see [101])

$$
\left(\mathrm{B}_{\mathrm{Ob}}^{(2 \otimes)} \otimes \mathrm{id}\right) \circ\left(\mathrm{id} \otimes \mathrm{B}_{\mathrm{Ob}}^{(2 \otimes)}\right) \circ\left(\mathrm{B}_{\mathrm{Ob}}^{(2 \otimes)} \otimes \mathrm{id}\right)=\left(\mathrm{id} \otimes \mathrm{B}_{\mathrm{Ob}}^{(2 \otimes)}\right) \circ\left(\mathrm{B}_{\mathrm{Ob}}^{(2 \otimes)} \otimes \mathrm{id}\right) \circ\left(\mathrm{id} \otimes \mathrm{B}_{\mathrm{Ob}}^{(2 \otimes)}\right) .
$$

If the braided semigroupal category bSGCat ${ }_{2}$ contains a unit object, then we have

Definition 42. A (binary) braided monoidal category

$$
\operatorname{MonCat}_{2}\left(\mathscr{C}, \mathcal{M}^{(2 \otimes)}, \mathrm{A}^{(3 \otimes)}, E, \mathrm{U}^{(2 \otimes)}, \mathrm{B}^{(2 \otimes)}\right)
$$

is the category bSGCat $_{2}$ together with a unit object $E \in \mathrm{Ob} \mathscr{C}$ satisfying the triangle axiom (111) and a unitor $\mathrm{U}^{(2 \otimes)}(109)$ and (110) the compatibility condition with the braiding $\mathrm{B}^{(2 \otimes)}$ such that the diagram (for objects)

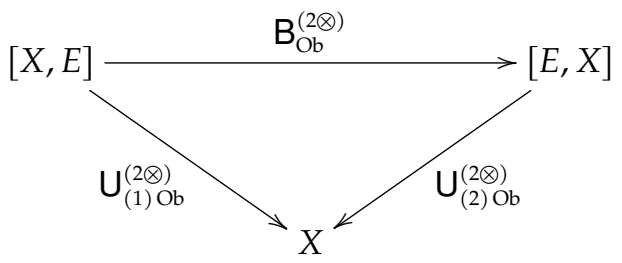

commutes.

For more details on binary braided monoidal categories, see $[17,102]$ and for review, see, e.g., $[14,103,104]$.

\subsection{Braided Polyadic Tensor Categories}

Higher braidings for binary tensor categories were considered (from an $n$-category viewpoint) in $[93,105]$. We will discuss them for polyadic categories, defined above in Section 8. The difference will be clearer if a polyadic category is not arity-reduced (see Definition 30) and for non-unital groupal categories (Section 9.2).

Let $\left(\mathscr{C}, \mathcal{M}^{(n \otimes)}, \mathrm{A}^{(2 n-1) \otimes}\right)$ be a polyadic non-strict semigroupal category, where $\mathcal{M}^{(n \otimes)}$ is a (not arity reduced) $n$-ary tensor product (n-ary functor) and $\mathbf{A}^{(2 n-1) \otimes}$ is an associator, i.e., $n-1$ different $(2 n-1)$-ary natural transformations (see Definition 32). Now the braiding becomes an $n$-ary natural transformation, which leads to any of $n$ permutations from the symmetry (permutation) group $S_{n}$ rather than one possibility only, as for the binary braiding (136). Note that in the consideration of higher braidings $[93,105]$ one ("order reversing") element of $S_{n}$ was used $\sigma_{n}^{(\text {rev })} \equiv\left(\begin{array}{cccc}1 & 2 & \ldots & n \\ n & n-1 & \ldots & 1\end{array}\right) \in S_{n}$. Thus, we arrive at the most general

Definition 43. An n-ary braiding $\mathrm{B}^{n \otimes}=\left\{\mathrm{B}_{\mathrm{Ob}}^{(n \otimes)}, \mathrm{B}_{\mathrm{Mor}}^{(n \otimes)}\right\}$ of a polyadic non-strict semigroupal category is an n-ary natural (or infranatural) transformation

$$
\mathrm{B}_{\mathrm{Ob}}^{(n \otimes)}: \mathcal{M}_{\mathrm{Ob}}^{(n \otimes)}[\mathbf{X}] \stackrel{\simeq}{\rightrightarrows} \mathcal{M}_{\mathrm{Ob}}^{(n \otimes)}\left[\sigma_{n} \circ \mathbf{X}\right],
$$

where $\mathbf{X}$ is an X-polyad (see Definition 31) of the necessary length (which is $n$ here), and $\sigma_{n} \in S_{n}$ are permutations that may satisfy some consistency conditions. The action on morphisms $\mathrm{B}_{\text {Mor }}^{(n \otimes)}$ may be found from the corresponding diagonal of the natural transformation square (cf. (98)). 
The binary non-mixed (standard) braiding (136) has $\sigma_{2}=\sigma_{2}^{(r e v)}=\left(\begin{array}{ll}1 & 2 \\ 2 & 1\end{array}\right) \in S_{2}$.

Definition 44. A polyadic (non-strict) semigroupal category endowed with the n-ary braiding $\left(\mathscr{C}, \mathcal{M}^{(n \otimes)}, \mathrm{A}^{(2 n-1) \otimes}, \mathrm{B}^{(n \otimes)}\right)$ is called a braided semigroupal polyadic category bSGCat $_{n}$.

The $n$-ary braiding $\mathrm{B}^{(n \otimes)}$ is connected with the associator $\mathrm{A}^{(2 n-1) \otimes}$ by a polyadic analog of the hexagon identity (137).

Example 8. In the case $n=3$, the braided non-strict semigroupal ternary category bSGCat 3 contains two associators $\mathrm{A}_{1}^{(5 \otimes)}$ and $\mathrm{A}_{2}^{(5 \otimes)}$ (see Example 5) satisfying the decagon axiom (124). Let us take for the ternary braiding $\mathrm{B}^{(3 \otimes)}$ its "order reversing" version

$$
\mathrm{B}_{\mathrm{Ob}}^{(3 \otimes)}: \mathcal{M}_{\mathrm{Ob}}^{(2 \otimes)}\left[X_{1}, X_{2}, X_{3}\right] \stackrel{\simeq}{\rightarrow} \mathcal{M}_{\mathrm{Ob}}^{(2 \otimes)}\left[X_{3}, X_{2}, X_{1}\right], \quad \forall X_{i} \in \mathrm{Ob} \mathscr{C}, \quad i=1,2,3 .
$$

Then the ternary analog of the hexagon identity is the decagon identity such that the diagram

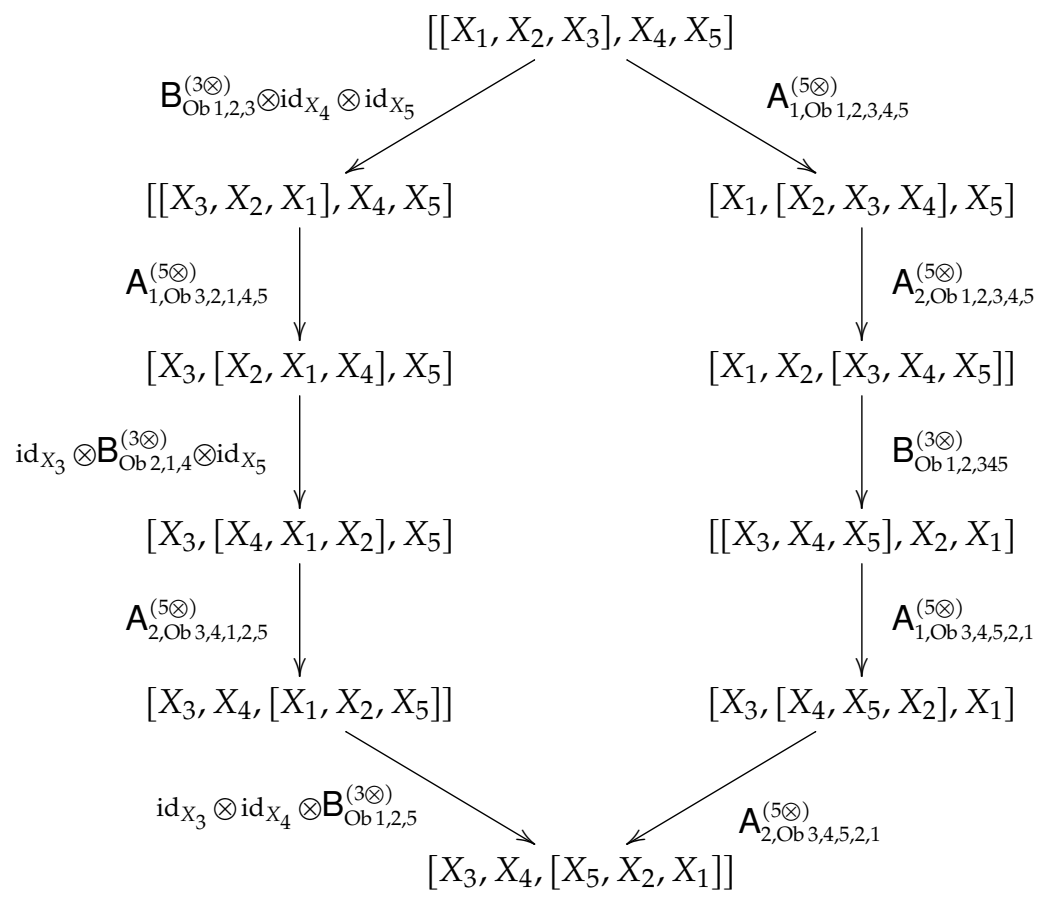

commutes.

Conjecture 4 (Braided $n$-ary coherence). If the $n$-ary associator $\mathrm{A}^{(2 n-1) \otimes}$ satisfies such $n$-ary coherence conditions that the isomorphism (123) takes place, and the $n$-ary braiding $\mathrm{B}^{(n \otimes)}$ satisfies the polyadic analog of the hexagon identity, then any diagram containing $\mathrm{A}^{(2 n-1) \otimes}$ and $\mathrm{B}^{(n \otimes)}$ commutes.

Proposition 15. If the braided semigroupal ternary category bSGCat 3 is strict (the associators becomes equivalences, and we can omit internal brackets), then the diagram containing only the ternary braidings $\mathrm{B}^{(3 \otimes)}$ 


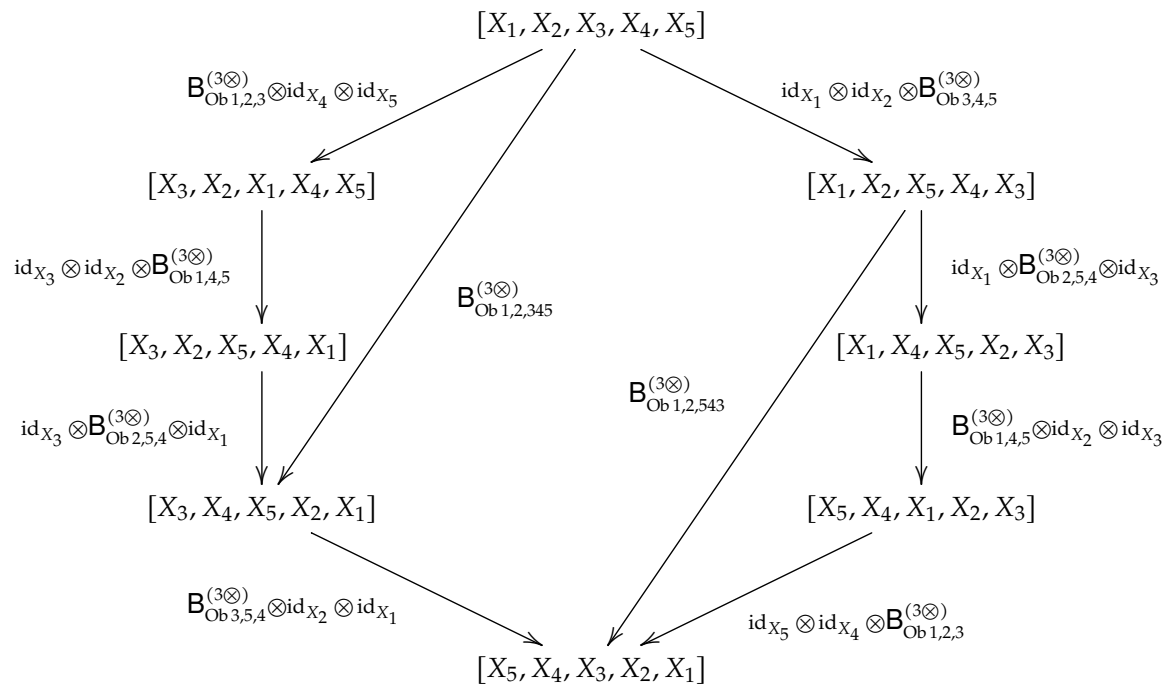

commutes (cf. the binary braiding (141)).

Proof. This is analogous to (141).

There follows from (147), omitting indices, the ternary braid group relation in terms of tensor products (cf. the tetrahedron equation $[93,106,107]$ )

$$
\begin{aligned}
& \left(\mathrm{B}^{(3 \otimes)} \otimes \mathrm{id} \otimes \mathrm{id}\right) \circ\left(\mathrm{id} \otimes \mathrm{B}^{(3 \otimes)} \otimes \mathrm{id}\right) \circ\left(\mathrm{id} \otimes \mathrm{id} \otimes \mathrm{B}^{(3 \otimes)}\right) \circ\left(\mathrm{B}^{(3 \otimes)} \otimes \mathrm{id} \otimes \mathrm{id}\right) \\
& =\left(\mathrm{id} \otimes \mathrm{id} \otimes \mathrm{B}^{(3 \otimes)}\right) \circ\left(\mathrm{B}^{(3 \otimes)} \otimes \mathrm{id} \otimes \mathrm{id}\right) \circ\left(\mathrm{id} \otimes \mathrm{B}^{(3 \otimes)} \otimes \mathrm{id}\right) \circ\left(\mathrm{id} \otimes \mathrm{id} \otimes \mathrm{B}^{(3 \otimes)}\right),
\end{aligned}
$$

which was obtained in [9] using another approach: by the associative quiver technique from [38]. For instance, the 4-ary braid group relation for 4-ary braiding $\mathrm{B}^{(4 \otimes)}$ has the form

$$
\begin{aligned}
& \left(\mathrm{B}^{(4 \otimes)} \otimes \mathrm{id} \otimes \mathrm{id} \otimes \mathrm{id}\right) \circ\left(\mathrm{id} \otimes \mathrm{B}^{(4 \otimes)} \otimes \mathrm{id} \otimes \mathrm{id}\right) \circ\left(\mathrm{id} \otimes \mathrm{id} \otimes \mathrm{B}^{(4 \otimes)} \otimes \mathrm{id}\right) \\
& \circ\left(\mathrm{id} \otimes \mathrm{id} \otimes \mathrm{id} \otimes \mathrm{B}^{(4 \otimes)}\right) \circ\left(\mathrm{B}^{(4 \otimes)} \otimes \mathrm{id} \otimes \mathrm{id} \otimes \mathrm{id}\right)=\left(\mathrm{id} \otimes \mathrm{id} \otimes \mathrm{id} \otimes \mathrm{B}^{(4 \otimes)}\right) \\
& \circ\left(\mathrm{B}^{(4 \otimes)} \otimes \mathrm{id} \otimes \mathrm{id} \otimes \mathrm{id}\right) \circ\left(\mathrm{id} \otimes \mathrm{id} \otimes \mathrm{B}^{(4 \otimes)} \otimes \mathrm{id}\right) \circ\left(\mathrm{id} \otimes \mathrm{B}^{(4 \otimes)} \otimes \mathrm{id} \otimes \mathrm{id}\right) \\
& \circ\left(\mathrm{id} \otimes \mathrm{id} \otimes \mathrm{id} \otimes \mathrm{B}^{(4 \otimes)}\right) .
\end{aligned}
$$

For the non-mixed "order reversing" n-ary braiding (see Definition 43) we have [9]. 
Proposition 16. The $n$-ary braid equation contains $(n+1)$ multipliers, and each one acts on $(2 n-1)$ tensor products as

$$
\begin{aligned}
& (\mathrm{B}^{(n \otimes)} \otimes \overbrace{\mathrm{id} \otimes \ldots \otimes \mathrm{id}}^{n-1}) \circ(\mathrm{id} \otimes \mathrm{B}^{(n \otimes)} \otimes \overbrace{\mathrm{id} \otimes \ldots \otimes \mathrm{id}}^{n-2}) \circ(\mathrm{id} \otimes \mathrm{id} \otimes \mathrm{B}^{(n \otimes)} \otimes \overbrace{\mathrm{id} \otimes \ldots \otimes \mathrm{id}}^{n-3}) . \\
& \circ(\overbrace{\left.\mathrm{id} \otimes \ldots \otimes \mathrm{id} \otimes \mathrm{B}^{(n \otimes)} \otimes \mathrm{id}\right) \circ(\overbrace{\mathrm{id} \otimes \ldots \otimes \mathrm{id} \otimes \mathrm{B}^{(n \otimes)}}^{n-2}) \circ(\mathrm{B}^{(n \otimes)} \otimes \overbrace{\mathrm{id} \otimes \ldots \otimes \mathrm{id}}^{n-1})}^{n-1}) \\
& =(\overbrace{\mathrm{id} \otimes \ldots \otimes \mathrm{id} \otimes \mathrm{B}^{(n \otimes)}}^{n-1}) \circ(\mathrm{B}^{(n \otimes)} \otimes \overbrace{\mathrm{id} \otimes \ldots \otimes \mathrm{id}}^{n-1}) \circ(\mathrm{id} \otimes \mathrm{B}^{(n \otimes)} \otimes \overbrace{\mathrm{id} \otimes \ldots \otimes \mathrm{id}}^{n-2}) \ldots \\
& \circ(\overbrace{\left.\mathrm{id} \otimes \ldots \otimes \mathrm{id} \otimes \mathrm{B}^{(n \otimes)} \otimes \mathrm{id} \otimes \mathrm{id}\right) \circ(\overbrace{\mathrm{id} \otimes \ldots \otimes \mathrm{id} \otimes \mathrm{B}^{(n \otimes)} \otimes \mathrm{id}}^{n-3})}^{n-2}) \\
& \circ(\overbrace{\mathrm{id} \otimes \ldots \otimes \mathrm{id} \otimes \mathrm{B}^{(n \otimes)}}^{n-1}) .
\end{aligned}
$$

Remark 4. If a polyadic category is arity-nonreducible, then the higher n-ary braid relations cannot be "iterated", i.e., obtained from the lower $n$ ones.

Consider a polyadic monoidal category MonCat ${ }_{n}$ with one unit object $E$ (see Definition 35). Then the $n$-ary braiding $\mathrm{B}^{(n \otimes)}$ satisfies the triangle identity connecting it with the unitors $\mathrm{U}^{(n \otimes)}$.

Example 9. In the case of the ternary monoidal category MonCat 3 (see Example 6) the "order reversing" braiding $\mathrm{B}^{(3 \otimes)}(145)$ satisfies an additional triangle identity analogous to (143) such that the diagram

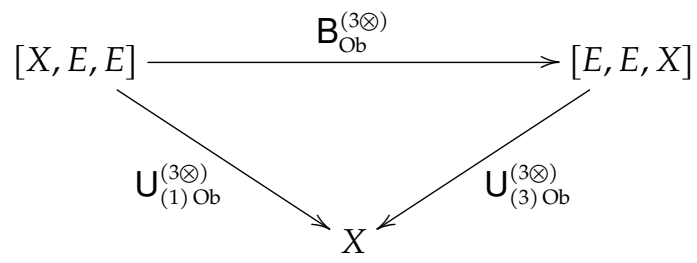

commutes.

For the polyadic non-unital groupal category GCat $_{n}$ (see Definition 37 ) the $n$-ary braiding $\mathrm{B}^{(n \otimes)}$ should be consistent with the quertors $\mathrm{U}^{(n \otimes)}$ and the querfunctor $\mathcal{Q}$ (see Definition 36).

Definition 45. A braided polyadic groupal category bGCat $_{n}$ is a polyadic groupal category GCat ${ }_{n}$ endowed with the $n$-ary braiding $\left(\mathscr{C}, \mathcal{M}^{(n \otimes)}, \mathrm{A}^{(2 n-1) \otimes}, \mathcal{Q}, \mathrm{Q}^{(n \otimes)}, \mathrm{B}^{(n \otimes)}\right)$. 
Example 10. In the ternary groupal category $\mathrm{GCat}_{3}$ (see Example 7) the "order reversing" braiding $\mathrm{B}^{(3 \otimes)}$ (145) satisfies the additional identity of consistency with the querfunctor 2 and quertor $\mathrm{Q}^{(3 \otimes)}$ such that the diagram

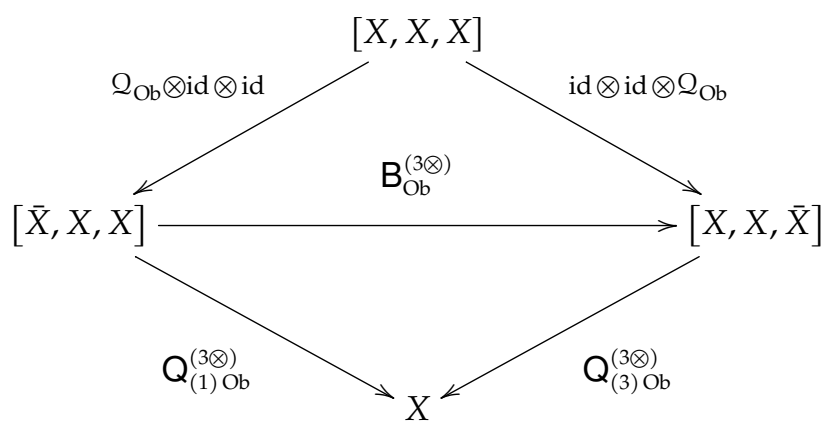

commutes.

The above diagrams ensure that some version of coherence can also be proven for braided polyadic categories.

\section{Medialed Polyadic Tensor Categories}

Here we consider a medial approach to braiding inspired by the first part of our paper. As opposed to binary braiding which is defined by one unique permutation (136), the $n$-ary braiding can be defined by the enormous number of possible allowed permutations (144). Therefore, in most cases only one permutation, that is the "order reversing", is usually (and artificially) used (see, e.g., [105]) ignoring other possible cases. On the other side, for $n$-ary structures it is natural to use the mediality property (54) which is unique in the $n$-ary case and for binary groups reduces to commutativity. So we introduce a medialing instead of braiding for the tensor product in categories, and (by analogy with braided categories) we call them medialed categories.

Let $\left(\mathscr{C}, \mathcal{M}^{(n \otimes)}, \mathrm{A}^{(2 n-1) \otimes}\right)$ be a polyadic non-strict semigroupal category SGCat $_{n}$ (see Definition 32).

Definition 46. An n-ary medialing $\left.\mathrm{M}^{\left(n^{2} \otimes\right.}\right)$ (or "medial braiding") is a mediality constraint which is a natural (or infranatural) transformation of two composed n-ary tensor product functors $\mathcal{M}^{(n \otimes)}$ (or functorial $n^{2}$-isomorphism)

$$
\mathrm{M}_{\mathrm{Ob}}^{\left(n^{2} \otimes\right)}: \mathcal{M}_{\mathrm{Ob}}^{(n \otimes)}\left[\begin{array}{c}
\mathcal{M}_{\mathrm{Ob}}^{(n \otimes)}\left[X_{11}, X_{12}, \ldots, X_{1 n}\right], \\
\mathcal{M}_{\mathrm{Ob}}^{(n \otimes)}\left[X_{21}, X_{22}, \ldots, X_{2 n}\right], \\
\vdots \\
\mathcal{M}_{\mathrm{Ob}}^{(n \otimes)}\left[X_{n 1}, X_{n 2}, \ldots, X_{n n}\right]
\end{array}\right] \stackrel{\cong}{\mathcal{M}_{\mathrm{Ob}}^{(n \otimes)}}\left[\begin{array}{c}
\mathcal{M}_{\mathrm{Ob}}^{(n \otimes)}\left[X_{11}, X_{21}, \ldots, X_{n 1}\right] \\
\mathcal{M}_{\mathrm{Ob}}^{(n \otimes)}\left[X_{12}, X_{22}, \ldots, X_{n 2}\right] \\
\vdots \\
\mathcal{M}_{\mathrm{Ob}}^{(n \otimes)}\left[X_{1 n}, X_{2 n}, \ldots, X_{n n}\right]
\end{array}\right],
$$

where the action on morphisms $\mathcal{M}_{\mathrm{Mor}}^{(n \otimes)}$ can be viewed as the corresponding diagonal in the natural transformation diagram as in (98).

Remark 5. The advantage of n-ary medialing is its uniqueness, because it does not contain a huge number of possible permutations $\sigma_{n} \in S_{n}$ as does the n-ary braiding (144).

Example 11. In the binary case $n=2$ we have (using the standard notation $\left.\mathcal{M}^{(2 \otimes)} \longrightarrow \otimes\right)$

$$
\mathrm{M}_{\mathrm{Ob}}^{(4 \otimes)}:\left(X_{1} \otimes X_{2}\right) \otimes\left(X_{3} \otimes X_{4}\right) \stackrel{\simeq}{\rightarrow}\left(X_{1} \otimes X_{3}\right) \otimes\left(X_{2} \otimes X_{4}\right), \forall X_{i} \in \mathrm{Ob} \mathscr{C}, i=1, \ldots, 4,
$$

which is called a binary medialing by analogy with binary braiding (136). 
In the compact matrix notation (see Definition 16) instead of (153) we have (symbolically)

$$
\mathrm{M}_{\mathrm{Ob}}^{\left(n^{2} \otimes\right)}:\left(\mathcal{M}_{\mathrm{Ob}}^{(n \otimes)}\right)^{2}\left[\hat{\boldsymbol{X}}_{\left(n^{2}\right)}\right] \stackrel{\sim}{\rightarrow}\left(\mathcal{M}_{\mathrm{Ob}}^{(n \otimes)}\right)^{2}\left[\hat{\boldsymbol{X}}_{\left(n^{2}\right)}^{T}\right],
$$

where the matrix polyads of objects is (cf. (57))

$$
\hat{\boldsymbol{X}}_{\left(n^{2}\right)}=\left(\mathrm{X}_{i j}\right) \in(\mathrm{Ob} \mathscr{C})^{\otimes n^{2}}, \quad X_{i j} \in \mathrm{Ob} \mathscr{C},
$$

and ()$^{T}$ is matrix transposition.

Definition 47. A medialed polyadic semigroupal category

$$
\operatorname{mSGCat}_{n}=\left(\mathscr{C}, \mathcal{M}^{(n \otimes)}, \mathrm{A}^{(2 n-1) \otimes}, \mathrm{M}^{\left(n^{2} \otimes\right)}\right)
$$

is a polyadic non-strict semigroupal category $\mathrm{SGCat}_{n}$ (see Definition 34) endowed with the n-ary medialing $\mathrm{M}^{\left(n^{2} \otimes\right)}$ satisfying the $n$-ary medial coherence condition (a medial analog of the hexagon identity (137)).

Definition 48. A medialed polyadic monoidal category

$$
\operatorname{mMonCat}_{n}=\left(\mathscr{C}, \mathcal{M}^{(n \otimes)}, \mathrm{A}^{(2 n-1) \otimes}, E, \mathbf{U}^{(n \otimes)}, \mathbf{M}^{\left(n^{2} \otimes\right)}\right)
$$

is a medialed polyadic semigroupal category mSGCat $_{n}$ with the unit object $E \in \mathrm{Ob} \mathscr{C}$ and the unitor $\mathrm{U}^{(n \otimes)}$ satisfying some compatibility condition.

Let us consider the polyadic nonunital groupal category GCat ${ }_{n}$ (see Definition 37), then the $n$-ary medialing $\mathrm{M}^{\left(n^{2} \otimes\right)}$ should be consistent with the quertors $\mathrm{U}^{(n \otimes)}$ and the querfunctor $Q$ (see Definition 36 and also the consistency condition for the ternary braiding (152)).

Definition 49. A braided polyadic groupal category

$$
\operatorname{mGCat}_{n}=\left(\mathscr{C}, \mathcal{M}^{(n \otimes)}, \mathrm{A}^{(2 n-1) \otimes}, \mathrm{Q}, \mathrm{Q}^{(n \otimes)}, \mathrm{M}^{\left(n^{2} \otimes\right)}\right)
$$

is a polyadic groupal category $\mathrm{GCat}_{n}$ endowed with the $n$-ary medialing $\mathrm{M}^{\left(n^{2} \otimes\right)}$.

\section{Medialed Binary and Ternary Categories}

Due to the complexity of the relevant polyadic diagrams, it is not possible to draw them in a general case for arbitrary arity $n$. Therefore, it would be worthwhile to consider first the binary case, and then some of the diagrams for the ternary case.

Example 12. Let

$$
\mathrm{mSGCat}_{2}=\left(\mathscr{C}, \mathcal{M}^{(2 \otimes)}, \mathrm{A}^{(3 \otimes)}, \mathrm{M}^{(4 \otimes)}\right)
$$

be a binary medialed semigroupal category and the binary medialing be as in (154). Then the medial analog of the hexagon identity (137) is given by the binary medial coherence condition such that the diagram 

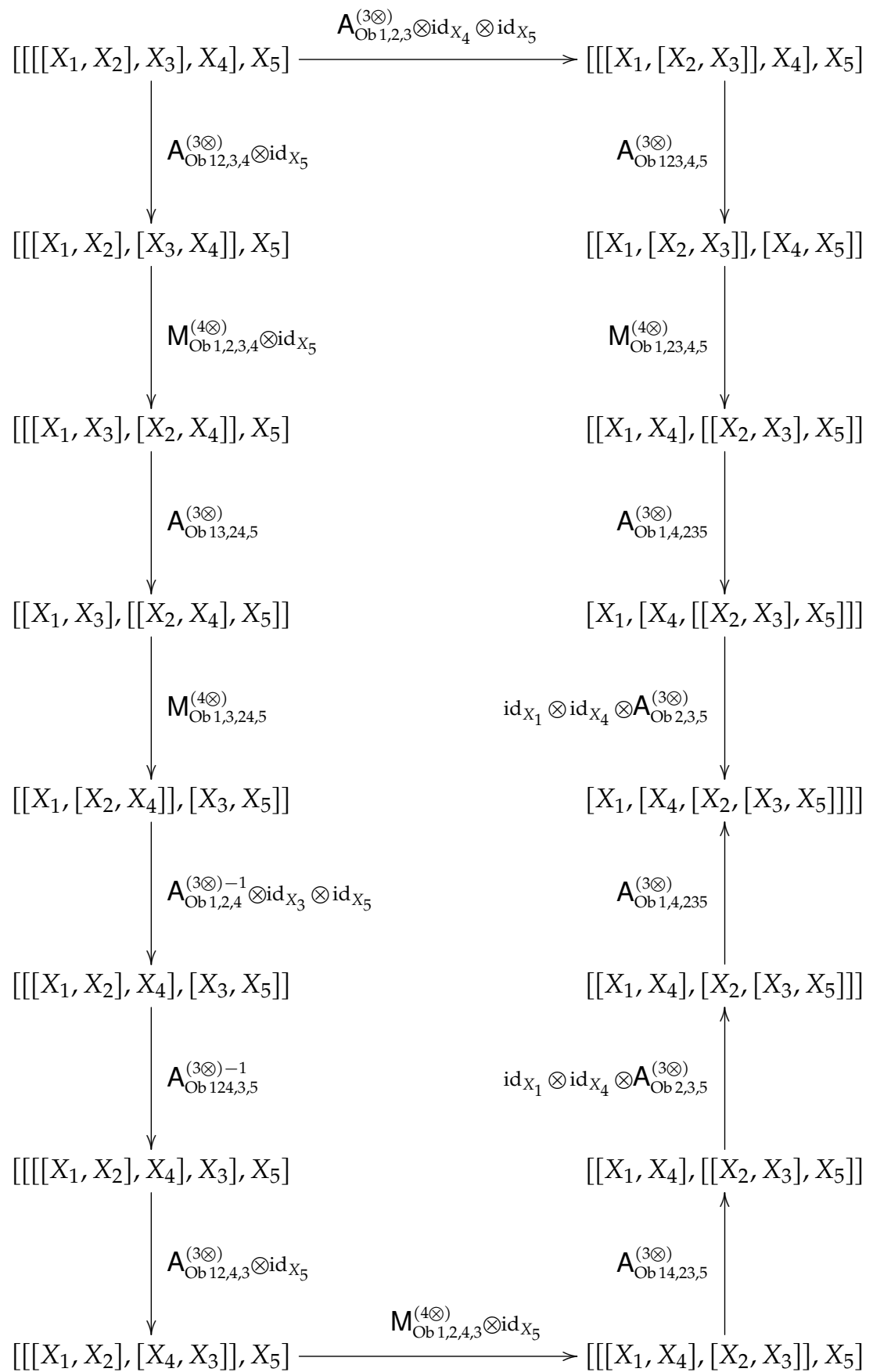

commutes.

If a medialed semigroupal category mSGCat 2 contains a unit object and the unitor, then we have

Definition 50. A medialed monoidal category

$$
\text { mMonCat }_{2}=\left(\mathscr{C}, \mathcal{M}^{(2 \otimes)}, \mathrm{A}^{(3 \otimes)}, E, \mathrm{U}^{(2 \otimes)}, \mathrm{M}^{(4 \otimes)}\right)
$$

is a (binary) medialed semigroupal category mSGCat $_{2}$ together with a unit object $E \in \mathrm{Ob} \mathscr{C}$ and a unitor $\mathrm{U}^{(2 \otimes)}(109)$ and (110) satisfying the triangle axiom (111). 
For mMonCat 2 the compatibility condition of the medialing $\mathrm{M}^{(4 \otimes)}$ with $E$ and $\mathrm{U}^{(2 \otimes)}$ is given by the commutative diagram

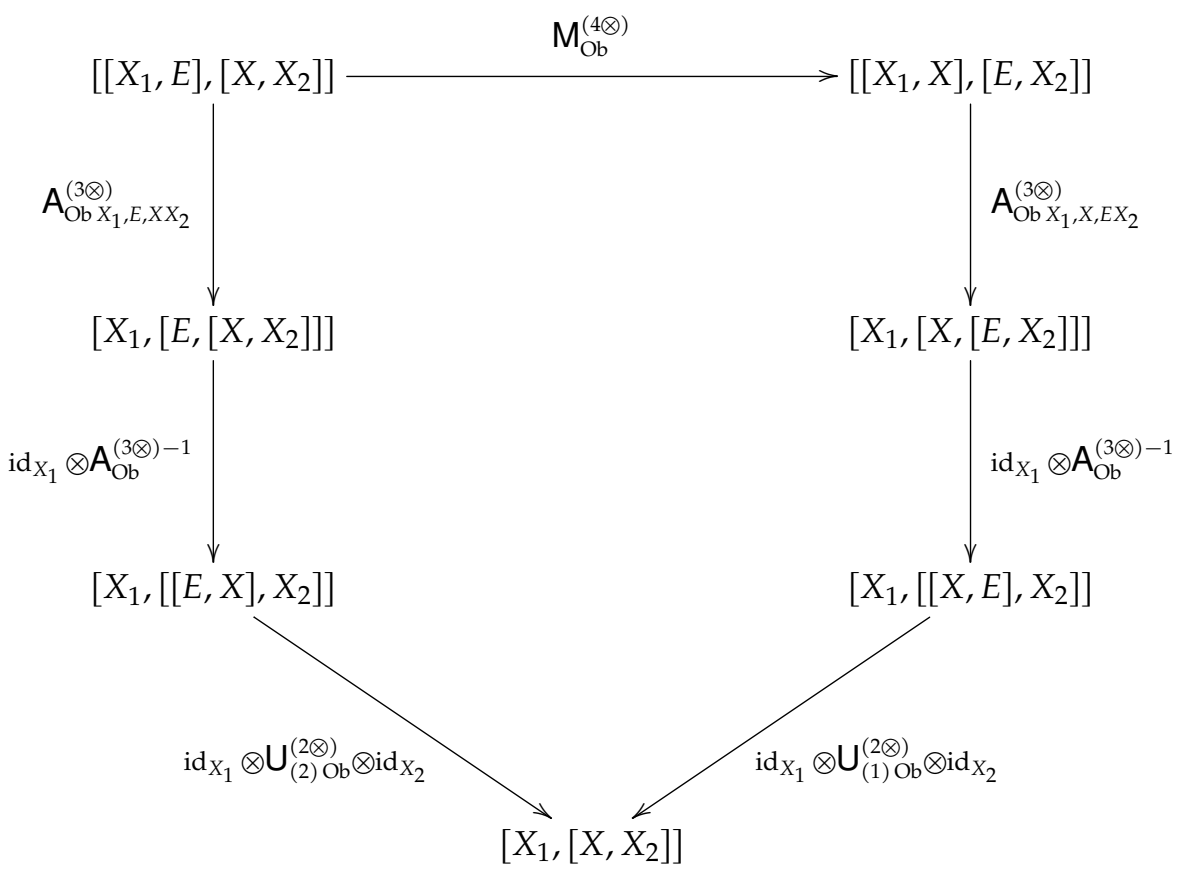

which is an analog of the triangle diagram for braiding (143).

Example 13. In the ternary nonunital groupal category $\mathrm{GCat}_{3}$ (see Example 7) the medialing $\mathrm{M}^{(9 \otimes)}$ satisfies the additional identity of consistency with the querfunctor $\mathcal{Q}$ and quertor $\mathrm{Q}^{(3 \otimes)}$ such that the diagram 


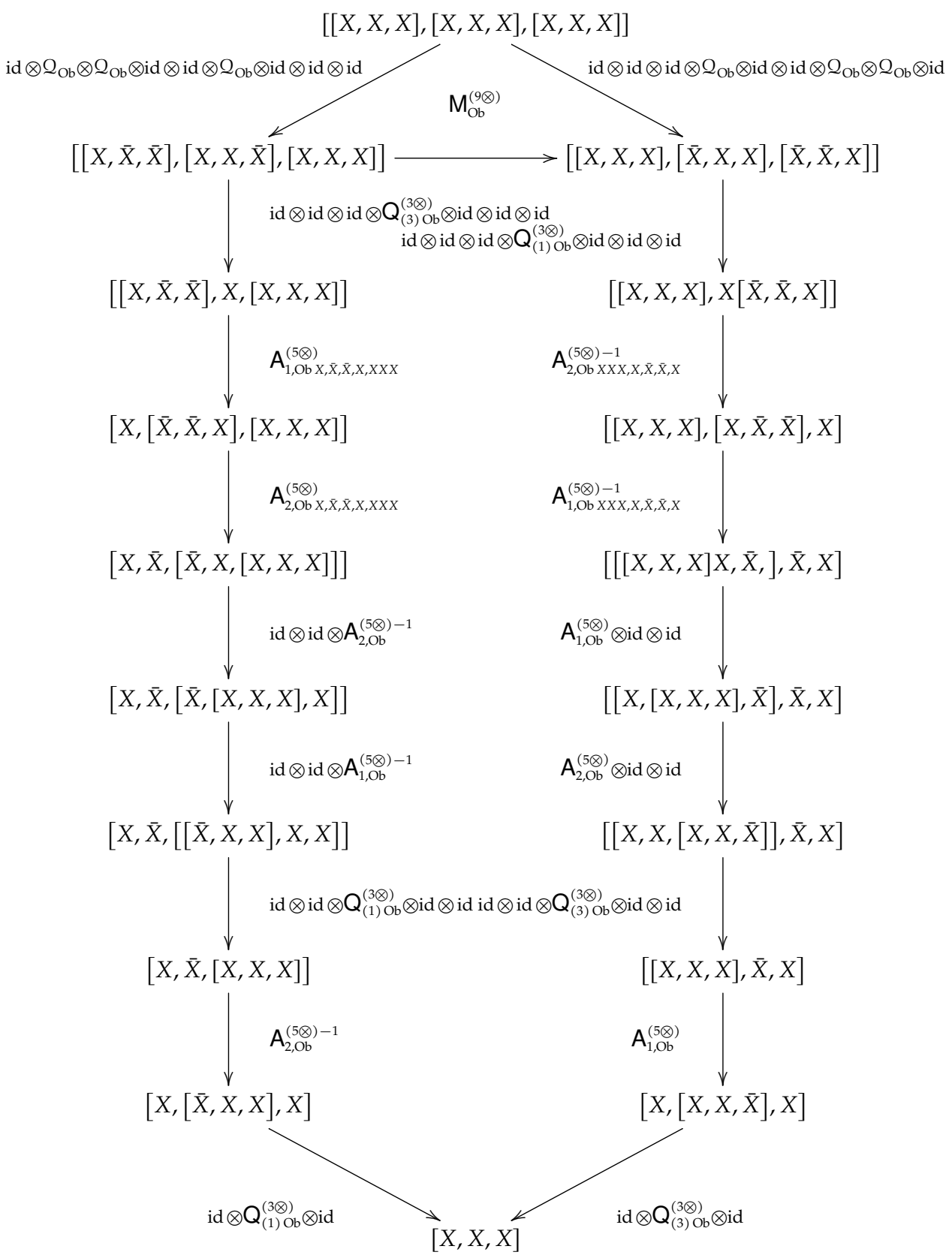

(159)

commutes. An analog of the hexagon identity in $\mathrm{GCat}_{3}$ can be expressed by a diagram which is similar to (157).

\section{Conclusions}

Commutativity in polyadic algebraic structures is defined non-uniquely, if one considers permutations and their combinations. We proposed a canonical way out: to substitute the commutativity property by mediality. Following this "commutativity-to-mediality" ansatz, we first investigated mediality for graded linear $n$-ary algebras and arrived at the concept of almost mediality, which is an analog of almost commutativity. We constructed "deforming" medial brackets, which could be treated as a medial analog of Lie brackets. We then proved Toyoda's theorem for almost medial $n$-ary algebras. Inspired by the above as examples, we proposed generalizing tensor and braided categories in a similar way. We defined polyadic tensor categories with an additional $n$-ary tensor multiplication for which a polyadic analog of the pentagon axiom was given. Instead of braiding we introduced $n$-ary "medialing" which satisfies a medial analog of the hexagon identity and constructed 
the "medialed" polyadic version of tensor categories. More details and examples will be presented in a forthcoming paper.

Funding: This research received no external funding.

Acknowledgments: The author would like to express his deep thankfulness to Vladimir Akulov, Andrew Bruce, Grigorij Kurinnoj, Mike Hewitt, Richard Kerner, Maurice Kibler, Dimitry Leites, Yuri Manin, Thomas Nordahl, Valentin Ovsienko, Norbert Poncin, Jim Stasheff, Vladimir Tkach, Raimund Vogl, Alexander Voronov and Wend Werner for numerous fruitful discussions and valuable support.

Conflicts of Interest: The author declares no conflict of interest.

\section{References}

1. Murdoch, D.C. Quasi-groups which satisfy certain generalized associative laws. Am. J. Math. 1939, 61, 509-522. [CrossRef]

2. Rittenberg, V.; Wyler, D. Generalized superalgebras. Nucl. Phys. B 1978, 139, 189-202. [CrossRef]

3. Scheunert, M. Generalized Lie algebras. J. Math. Phys. 1979, 20, 712-720. [CrossRef]

4. Bahturin, Y.; Montgomery, S.; Zaicev, M. Generalized Lie solvability of associative algebras. In Groups, Rings, Lie and Hopf Algebras; Bahturin, Y., Ed.; Kluwer: Dordrecht, The Netherands, 2003; pp. 1-23.

5. Bongaarts, P.J.M.; Pijls, H.G.J. Almost commutative algebra and differential calculus on the quantum hyperplane. J. Math. Phys. 1994, 35, 959-970. [CrossRef]

6. Covolo, T.; Michel, J.P. Determinants over graded-commutative algebras, a categorical viewpoint. L'Enseign. Math. 2016, 62, 361-420. [CrossRef]

7. Morier-Genoud, S.; Ovsienko, V. Simple graded commutative algebras. J. Algebra 2010, 323, 1649-1664. [CrossRef]

8. Toyoda, K. On axioms of linear functions. Proc. Imp. Acad. Tokyo 1941, 17, 221-227. [CrossRef]

9. Duplij, S. Polyadic Hopf algebras and quantum groups. East European J. Phys. 2021, 2, 5-50. [CrossRef]

10. Dalzotto, G.; Sbarra, E. On non-standard graded algebras. Toyama Math. J. 2008, 31, 33-57.

11. Elduque, A. A Lie grading which is not a semigroup grading. Linear Alg. Appl. 2006, 418, 312-314. [CrossRef]

12. Nystedt, P. Noncommutatively Graded Algebras. arxiv 2017, arXiv:1710.11400.

13. MacLane, S. Categories for the Working Mathematician; Springer: Berlin, Germany, 1971; p. 189.

14. Etingof, P.; Gelaki, S.; Nikshych, D.; Ostrik, V. Tensor Categories; Mathematische Zeitschrift: Providence, RI, USA, 2015. [CrossRef]

15. Dörnte, W. Unterschungen über einen verallgemeinerten Gruppenbegriff. Math. Z. 1929, 29, 1-19. [CrossRef]

16. Post, E.L. Polyadic groups. Trans. Am. Math. Soc. 1940. 48, 208-350. [CrossRef]

17. Joyal, A.; Street, R. Braided tensor categories. Adv. Math. 1993, 102, 20-78. [CrossRef]

18. Bourbaki, N. Algebra I: Chapters 1-3; Springer: Berlin, Germany, 1998.

19. Nastasescu, C.; Van Oystaeyen, F. Methods of Graded Rings; Springer: Berlin, Germany, 2004.

20. Duplij, S. Arity shape of polyadic algebraic structures. J. Math. Phys. Anal. Geom. 2019, 15, 3-56. [CrossRef]

21. Dade, E.C. Group-graded rings and modules. Math. Z. 1980, 174, 241-262. [CrossRef]

22. Aczél, J. On mean values. Bull. Am. Math. Soc. 1948, 54, 392-400. [CrossRef]

23. Ježek, J.; Kepka, T. Medial Groupoids; Academia: Praha, Czech Republic, 1983.

24. Evans, T. Abstract mean values. Duke Math. J. 1963, 30, 331-347. [CrossRef]

25. Howroyd, T. Cancellative medial groupoids and arithmetic means. Bull. Am. Math. Soc. 1973, 8, 17-21. [CrossRef]

26. Sholander, M. On the existence of the inverse operation in alternation groupoids. Bull. Am. Math. Soc. 1949, 55, 746-757. [CrossRef]

27. Ježek, J.; Kepka, T. A note on medial division groupoids. Proc. Am. Math. Soc. 1993, 119, 423-426. [CrossRef]

28. Shcherbacov, V. Elements of Quasigroup Theory and Applications; CRC Press: Boca Raton, FL, USA, 2017; p. xxi+576. [CrossRef]

29. Bruck, R.H. Some results in the theory of quasigroups. Trans. Am. Math. Soc. 1944. 55, 19-52. [CrossRef]

30. Murdoch, D.C. Structure of abelian quasi-groups. Trans. Am. Math. Soc. 1941, 49, 392-409. [CrossRef]

31. Chrislock, J.L. On medial semigroups. J. Alg. 1969, 12, 1-9. [CrossRef]

32. Nordahl, T.E. Medial Semigroups. Ph.D. Thesis, University of California, Davis, CA, USA, 1974.

33. Nagy, A. Special Classes of Semigroups; Kluwer: Dordrecht, The Netherlands, 2001; p. viii+269. [CrossRef]

34. Głazek, K.; Gleichgewicht, B. Abelian n-groups. In Universal Algebra (Esztergom, 1977); North-Holland: Amsterdam, The Netherlands; New York, NY, USA, 1982; Volume 29, pp. 321-329.

35. Dog, S. On entropicity in n-ary semigroups. Quasigroups Relat. Syst. 2016, 24, 197-204.

36. Michor, P.W.; Vinogradov, A.M. n-Ary Lie and associative algebras. Rend. Sem. Mat. Univ. Pol. Torino 1996, 54, $373-392$.

37. Goze, M.; Goze, N.; Remm, E. n-Lie algebras. Afr. J. Math. Phys. 2010, 8, 17-28.

38. Duplij, S. Polyadic Algebraic Structures And Their Representations. In Exotic Algebraic and Geometric Structures in Theoretical Physics; Duplij, S., Ed.; Nova Publishers: New York, NY, USA, 2018; pp. 251-308.

39. Gnedbaye, A.V. Les algèbres k-aires et leurs opérades. C. R. Acad. Sci. Paris Sér. I 1995, 321, 147-152.

40. López Peña, J.; Panaite, F.; Van Oystaeyen, F. General twisting of algebras. Adv. Math 2007, 212, 315-337. [CrossRef]

41. Bénabou, J. Catégories avec multiplication. C. R. Acad. Sci. 1963, 256, 1887-1890. 
42. Mac Lane, S. Natural associativity and commutativity. Rice Univ. Stud. 1963, 49, $28-46$.

43. Tannaka, T. Über den Dualitätssatz der nichtkommutativen topologischen Gruppen. Tôhoku Math. J. 1939, 45, 1-12.

44. Calaque, D.; Etingof, P. Lectures on tensor categories.

IRMA Lect. Math. Theor. Phys. 2008, 12, 1-38.

45. Müger, M. Tensor categories: A selective guided tour. Rev. Union Mat. Argent. 2010, 51, 95-163.

46. Baez, J.C.; Dolan, J. Categorification. In Higher Category Theory; Getzler, E., Kapranov, M., Eds.; Mathematische Zeitschrift: Providence, RI, USA, 1998; Volume 230, pp. 1-36.

47. Baez, J.C.; Dolan, J. Higher-dimensional algebra III. n-categories and the algebra of opetopes. Adv. Math. 1998, 135, 145-206.

48. Adámek, J.; Herrlich, H.; Strecker, G.E. Abstract and Concrete Categories: The Joy of Cats; Wiley and Sons: New York, NY, USA, 1990; p. 507.

49. Borceux, F. Handbook of Categorical Algebra 1. Basic Category Theory; Encyclopedia of Mathematics and Its Applications; Cambridge University Press: Cambridge, UK, 1994; Volume 50, p. xvi+345.

50. Crane, L.; Yetter, D.N. Examples of categorification. Cah. Topol. Geom. Diff. Categ. 1998, 39, 3-25.

51. Aguiar, M.; Mahajan, S.A. Monoidal Functors, Species and Hopf Algebras; CRM Monograph Series; Mathematische Zeitschrift: Providence, RI, USA, 2010; Volume 29.

52. Yetter, D.N. Functorial Knot Theory. Categories of Tangles, Coherence, Categorical Deformations, and Topological Invariants; Series on Knots and Everything; World Scientific: River Edge, NJ, USA, 2001; Volume 26, p. iv+230.

53. Lu, X.; Ye, Y.; Hu, S. A graphical calculus for semi-groupal categories. Appl. Categor. Struct. 2019, 27, 163-197. [CrossRef]

54. Boyarchenko, M. Associativity Constraints in Monoidal Categories. Available online: http://www.math.uchicago.edu/ may/ TQFT/Boyarchenko\%20on\%20associativity.pdf (accessed on 2 April 2021).

55. Kock, J. Elementary remarks on units in monoidal categories. Math. Proc. Camb. Phil. Soc. 2008, 144, 53-76. [CrossRef]

56. Stasheff, J. H-Spaces from a Homotopy Point of View; Springer: Berlin, Germany, 1970.

57. Abramsky, S.; Coecke, B. Categorical quantum mechanics. In Handbook of Quantum Logic and Quantum Structures; Engesser, K., Gabbay, D.M., Lehmann, D., Eds.; Elsevier: Amsterdam, The Netherlands, 2008; Volume 2, pp. 261-324.

58. Elgueta, J. Cohomology and deformation theory of monoidal 2-categories. I. Adv. Math. 2004, 182, 204-277. [CrossRef]

59. Davydov, A. Nuclei of categories with tensor products. Theory Appl. Categ. 2007, 18, 440-472.

60. Kelly, G.M. On Mac Lane's conditions for coherence of natural associativities, commutativities, etc. J. Algebra 1964, 1, 397-402. [CrossRef]

61. Stasheff, J.D. Homotopy associativity of H-spaces. I, II. Trans. Am. Math. Soc. 1963, 108, 293-312. [CrossRef]

62. Joyce, W.P. Natural associativity without the pentagon condition. arXiv 2001, arXiv:math/0109088.

63. Kelly, G.M. Tensor products in categories. J. Algebra 1965, 2, 15-37. [CrossRef]

64. Kassel, C. Quantum Groups; Springer: New York, NY, USA, 1995; p. 531.

65. Joyal, A.; Kock, J. Coherence for weak units. Doc. Math. 2013, 18, 71-110.

66. Andrianopoulos, J. Remarks on units of skew monoidal categories. Appl. Categ. Struct. 2017, 25, 863-873. [CrossRef]

67. Baez, J.C.; Dolan, J. Higher-dimensional algebra and topological quantum field theory. J. Math. Phys. 1995, 36, 6073-6105. [CrossRef]

68. Gal'mak, A.M. n-ary Groups, Part 1; Gomel University: Gomel, Russia, 2003; p. 195.

69. Crane, L.; Frenkel, I.B. Four-dimensional topological quantum field theory, Hopf categories, and the canonical bases. J. Math. Phys. 1994, 35, 5136-5154. [CrossRef]

70. Kelly, G.M.; Street, R. Review of the elements of 2-categories. In Category Seminar; Lecture Notes in Mathematics; Kelly, G.M., Ed.; Springer: Berlin/Heidelberg, Germany, 1974; Volume 420, pp. 75-103. [CrossRef]

71. Bénabou, J. Introduction to bicategories. In Reports of the Midwest Category Seminar; Springer: Berlin, Germany, $1967 ;$ pp. 1-77.

72. Baez, J.C. An Introduction to $n$-Categories. In Proceedings of the 7th Conference on Category Theory and Computer Science; Moggi, E., Rosolini, G., Eds.; Springer: Berlin, Germany, 1997; Volume 1290, pp. 1-33.

73. Leinster, T. A survey of definitions of $n$-category. Theory Appl. Categ. 2002, 10, 1-70.

74. Aldrovandi, E.; Noohi, B. Butterflies I: Morphisms of 2-group stacks. Adv. Math. 2009, 221, 687-773. [CrossRef]

75. Lambek. Deductive systems and categories III. In Category Theory, Homology Theory and Their Applications, I; Lecture Notes in Mathematics; Springer: Berlin, Germany, 1969; Volume 86, pp. 76-122.

76. Leinster, T. General Operads and Multicategories. arXiv 1998, arXiv:math/9810053.

77. Cruttwell, G.S.H.; Shulman, M.A. A unified framework for generalized multicategories. Theory Appl. Categ. $2010,24,580-655$.

78. Grandis, M. Higher Dimensional Categories: From Double to Multiple Categories; World Scientific: Singapore, 2020. [CrossRef]

79. Balteanu, C.; Fiedorowicz, Z.; Schwänzl, R.; Vogt, R. Iterated monoidal categories. Adv. Math. 2003, 176, 277-349. [CrossRef]

80. Cheng, E.; Gurski, N. Iterated icons. Theory Appl. Categ. 2014, 29, 929-977.

81. Duplij, S.; Marcinek, W. Regular Obstructed Categories and Topological Quantum Field Theory. J. Math. Phys. 2002, 43, 3329-3341. [CrossRef]

82. Duplij, S.; Marcinek, W. Higher Regularity And Obstructed Categories. In Exotic Algebraic and Geometric Structures in Theoretical Physics; Duplij, S., Ed.; Nova Publishers: New York, NY, USA, 2018; pp. 15-24.

83. Herrlich, H. A characterization of $k$-ary algebraic categories. Manuscr. Math. 1971, 4, 277-284. [CrossRef]

84. Shulman, M. Exact completions and small sheaves. Theory Appl. Categ. 2012, 27, 97-173. 
85. Wenninger, M.J. Polyhedron Models; Cambridge University Press: Cambridge, UK, 1974.

86. Pop, M.S.; Pop, A. On some relations on n-monoids. Carpathian J. Math. 2004, 20, 87-94.

87. Gleichgewicht, B.; Głazek, K. Remarks on n-groups as abstract algebras. Colloq. Math. 1967, 17, 209-219. [CrossRef]

88. Michalski, J. On some Functors from the Category of $n$-Groups. Bull. Acad. Polon. Sci. Ser. Sci. Math. 1979, 27, 345-349.

89. Michalski, J. On the category of $n$-groups. Fund. Math. 1984, 122, 187-197. [CrossRef]

90. Iancu, L. On the category of n-groups. Bul. Stiin. Univ. Baia Mare Seria B 1991, 7, 9-14.

91. Garner, R.; Franco, I.L. Commutativity. J. Pure Appl. Algebra 2016, 220, 1707-1751. [CrossRef]

92. Duplij, S.; Marcinek, W. Braid Semistatistics And Doubly Regular R-Matrix. In Exotic Algebraic and Geometric Structures in Theoretical Physics; Duplij, S., Ed.; Nova Publishers: New York, NY, USA, 2018; pp. 77-86.

93. Kapranov, M.; Voevodsky, V. Braided monoidal 2-categories and Manin-Schechtman higher braid groups. J. Pure Appl. Algebra 1994, 92, 241-267. [CrossRef]

94. Batanin, M. Locally constant $n$-operads as higher braided operads. J. Noncommut. Geom. 2010, 237-263. [CrossRef]

95. Weber, M. Operads within Monoidal Pseudo Algebras. Appl. Categ. Struct. 2005, 13, 389-420. [CrossRef]

96. von Neumann, J. On regular rings. Proc. Nat. Acad. Sci. USA 1936, 22, 707-713. [CrossRef]

97. Duplij, S.; Marcinek, W. Semisupermanifolds and regularization of categories, modules, algebras and Yang-Baxter equation. In Supersymmetry and Quantum Field Theory; Elsevier Science Publishers: Amsterdam, The Netherlands, $2001 ;$ pp. 110-115.

98. Penrose, R. A generalized inverse for matrices. Math. Proc. Camb. Phil. Soc. 1955, 51, 406-413. [CrossRef]

99. Nashed, M.Z. Generalized Inverses and Applications; Academic Press: New York, NY, USA, 1976; p. 321.

100. Drinfeld, V.G. Quasi-Hopf Algebras and Knizhnik-Zamolodchikov Equations. In Problems of Modern Quantum Field Theory; Belavin, A.A., Klimyk, A.V., Zamolodchikov, A.B., Eds.; Springer: Heidelberg, Germany, 1989; pp. 1-13.

101. Street, R. Higher categories, strings, cubes and simplex equations. Appl. Categ. Struct. 1995, 3, 29-77. [CrossRef]

102. Freyd, P.J.; Yetter, D.N. Braided compact closed categories with applications to low dimensional topology. Adv. Math. 1989, 77, 156-182. [CrossRef]

103. Chari, V.; Pressley, A. A Guide to Quantum Groups; Cambridge University Press: Cambridge, UK, 1996.

104. Bulacu, D.; Caenepeel, S.; Panaite, F.; Van Oystaeyen, F. Quasi-Hopf Algebras: A Categorical Approach; Cambridge University Press: Cambridge, UK, 2019. [CrossRef]

105. Manin, Y.I.; Schechtman, V. Arrangements of hyperplanes, higher braid groups and higher Bruhat orders. Adv. Stud. Pure Math. 1989, 17, 289-308.

106. Bazhanov, V.V.; Stroganov, Y.G. Conditions of commutativity of transfer matrices on a multidimensional lattice. Teor. Mat. Fiz. 1982, 52, 105-113. [CrossRef]

107. Baez, J.C.; Neuchl, M. Higher-Dimensional Algebra I: Braided Monoidal 2-Categories. Adv. Math. 1996, 121, 196-244. [CrossRef] 